TRANSACTIONS OF THE

AMERICAN MATHEMATICAL SOCIETY

Volume 328, Number 1, November 1991 .

\title{
COHOMOLOGY OF METACYCLIC GROUPS
}

\author{
JOHANNES HUEBSCHMANN
}

\begin{abstract}
Let e: $1 \rightarrow N \rightarrow G \rightarrow K \rightarrow 1$ be an extension of a finite cyclic group $N$ by a finite cyclic group $K$. Using homological perturbation theory, we introduce the beginning of a free resolution of the integers $Z$ over the group ring $\mathrm{Z} G$ of $G$ in such a way that the resolution reflects the structure of $G$ as an extension of $N$ by $K$, and we use this resolution to compute the additive structure of the integral cohomology of $G$ in many cases. We proceed by first establishing a number of special cases, thereafter constructing suitable cohomology classes thereby obtaining a lower bound, then computing characteristic classes introduced in an earlier paper, and, finally, exploiting these classes, obtaining upper bounds for the cohomology via the integral cohomology spectral sequence of the extension e. The calculation is then completed by comparing the two bounds.
\end{abstract}

\section{INTRODUCTION}

Few explicit integral calculations have been done in group cohomology. In particular, given a group extension $1 \rightarrow N \rightarrow G \rightarrow K \rightarrow 1$, even if the cohomologies of $N$ and $K$ are known, there is no general recipe to compute the cohomology of $G$ from the given data.

In the past years we developed methods to actually $d o$ such a calculation under appropriate circumstances. In [24] and [25] we offered means to handle nilpotent groups of class 2 . In the present paper we offer another such method; it consists of several steps: At first, one constructs cohomology classes of $G$ by means of standard methods such as, e.g., Chern classes of suitable representations and transfer. This provides a lower bound for the cohomology of $G$. The next step is to construct the beginning of a suitable free resolution for $G$ which arises from the tensor product of a free resolution for $N$ and $K$ by "homological perturbation theory". The natural framework for this construction is within differential homological algebra and will be explained in full detail elsewhere [28]. The beginning of a resolution is then used to compute certain characteristic classes which were introduced in an earlier paper [26]; these classes assume their values in certain Ext-groups and provide a description of

Received by the editors May 23, 1989 and, in revised form, January 11, 1990.

1980 Mathematics Subject Classification (1985 Revision). Primary 20J06, 18G40.

Key words and phrases. Cohomology of group extensions, cohomology of finite groups, homological perturbation theory.

The author was supported by the Deutsche Forschungsgemeinschaft under a Heisenberg grant. 
the first nonzero differential in the spectral sequence of the extension. The cohomology with respect to the first nonzero differential then provides an upper bound for the cohomology of $G$. In favorable circumstances the two bounds coincide. In the present paper we illustrate this philosophy by a computation of the integral cohomology of many metacyclic groups. For a prime $p$, the mod $p$ cohomology of such a group has been computed in our earlier paper [30]; in that paper the construction of the beginning of a free resolution has already been exploited. However, it is unclear whether integral calculations can be effectively carried out from these results and the Bockstein spectral sequence.

Thus, let $G$ be a metacyclic group, given by a presentation

$$
G=G(r, s, t, f)=\left\langle x, y ; y^{r}=1, x^{s}=y^{f}, x y x^{-1}=y^{t}\right\rangle,
$$

where $s>1, r>1, t^{s} \equiv 1 \bmod r, t f \equiv f \bmod r$. These conditions ensure that the group $G$ fits into a group extension

$$
\text { e: } 1 \rightarrow N \rightarrow G \rightarrow K \rightarrow 1,
$$

where $N=\left\langle y ; y^{r}=1\right\rangle$ and $K=\left\langle x ; x^{s}=1\right\rangle$. In the present paper we shall compute the additive structure of the integral cohomology of a large class of metacyclic groups. More precisely, for a number $m$, let $m_{p}$ denote the $p$ primary part, i.e., $m=m_{p} m^{\prime}$, where $m^{\prime}$ is not divisible by $p$. We shall compute the $p$-primary part of the integral cohomology of any metacyclic group $G(r, s, t, f)$ unless

$$
s_{p}>r_{p}, \quad f \not \equiv 0 \bmod r_{p}, \quad \text { and } t \not \equiv 1 \bmod f_{p} .
$$

To explain our result, we introduce the following notation:

$$
\begin{gathered}
h_{0}=r, \quad k_{0}=s, \quad q_{0}=s, \quad \nu=\frac{h_{1} f}{r}, \quad d=\text { order of } t \text { modulo } r, \\
h_{j}=\left(t^{j}-1, r\right), \quad k_{j}=\left(\sum_{\mu=0}^{s-1} t^{j \mu}, r\right), \quad q_{j}=\frac{h_{j} k_{j}}{r}, \quad j \geq 1,
\end{gathered}
$$

where as usual $(\cdots)$ refers to the greatest common divisor. We note that the second cohomology group $\mathrm{H}^{2}(K, N)$ has order $q_{1}$ and that $\nu$ modulo $q_{1}$ is the class in $\mathrm{H}^{2}(K, N)$ of the extension $\mathbf{e}$; moreover, for $j>1$, the numbers $q_{j}$ are divisible by $q_{1}$ (see (6.8) for details).

We shall say that a prime $p$ is bad for the presentation (0.1) if it divides $r$ and $s$, if $f \not \equiv 0 \bmod r_{p}$, and if $s_{p}>r_{p}$. It is not hard to see that a prime $p$ is bad for the presentation $(0.1)$ if and only if $\nu \not \equiv 0 \bmod \left(q_{1}\right)_{p}$ and if $s_{p}>r_{p}$ (see (6.12) below). A prime which is not bad for the presentation (0.1) will be said to be good for the presentation (0.1). Here is our main result.

Theorem 0.3. Given a presentation (0.1) of a metacyclic group $G$, there are classes $\zeta_{2 j} \in \mathrm{H}^{2 j}(G, \mathbf{Z})$ and $\xi_{2 j+1} \mathrm{H}^{2 j+1}(G, \mathbf{Z})$ so that, at each prime $p$ that is good for the presentation (0.1), as a graded module over the p-primary part 
of the integral cohomology $\mathrm{H}^{*}(\mathbf{Z} / s, \mathbf{Z})=\mathbf{Z}\left[c_{x}\right] / s c_{x}$ of the quotient $\mathbf{Z} / s$, the cohomology of $\mathrm{H}^{*}(G, \mathbf{Z})$ is generated by these classes subject to the relations

$$
\begin{aligned}
h_{j} \zeta_{2 j} & =\left(\nu, q_{1}\right) \frac{q_{j-1}}{q_{1}} c_{x} \zeta_{2 j-2}, \\
q_{j} c_{x} \zeta_{2 j} & =0, \\
\frac{q_{j}}{q_{1}}\left(\nu, q_{1}\right) c_{x}^{i} \zeta_{2 j} & =0, \quad i \geq 2,
\end{aligned}
$$

unless possibly $p=2, t \equiv-1 \bmod 4$, and $\nu$ and $j$ are odd, in which case

$$
c_{x}^{i} \zeta_{2 j}=\varepsilon \frac{q_{j-1}}{2 q_{1}} c_{x}^{i+1} \zeta_{2 j-2}, \quad i \geq 2,
$$

where $\varepsilon=0$ or $\varepsilon=1$, and, finally,

$$
\frac{q_{j}}{q_{1}}\left(\nu, q_{1}\right) \xi_{2 j+1}=0 .
$$

Moreover, as an algebra, the p-primary part of $\mathrm{H}^{*}(G, \mathbf{Z})$ is generated by the classes $\zeta_{2 j}$ and $\xi_{2 j+1}$ for $j \leq d_{p}$.

We note that in the split case $\left(\nu, q_{1}\right)=q_{1}$, the relations read

$$
\begin{aligned}
h_{j} \zeta_{2 j} & =0, \\
q_{j} c_{x} \zeta_{2 j} & =0, \\
q_{j} \xi_{2 j+1} & =0,
\end{aligned}
$$

and the relations

$$
q_{j} c_{x}^{i} \zeta_{2 j}=0, \quad i \geq 2,
$$

are consequences of the other ones. In this case our theorem yields precisely the dual of the additive structure of the homology of a split metacyclic group given by Wall [46]. Another special case is $t \equiv 1 \bmod r$ so that $G$ is abelian. In this case we have

$$
h_{j}=r, \quad k_{j}=(s, r), \quad q_{j}=k_{j}=(s, r), \quad \nu=f, \quad d=1,
$$

and we obtain formulas for the well-known structure of the cohomology of a finite abelian group with two generators.

In the above relations $(0.3 .3)$ there is an ambiguity at the prime 2 in case $t \equiv-1 \bmod 4$; however, we shall indicate in (8.27) below that the additive structure is yet still completely determined in this case. Thus our result gives in particular a complete description of the additive structure of the integral cohomology of those metacyclic groups for which every prime is good. There are of course many examples, and it is straightforward to decide whether or not a prime is good for a given presentation of a metacyclic group.

We now explain how these results are obtained: In $\S 2$ we settle the cases in which $G$ has an abelian or split metacyclic $p$-Sylow subgroup. This can be done 
by standard methods and Wall's [46] resolution. Thereafter it can be assumed that the $p$-Sylow subgroup of $G$ is neither abelian nor split metacyclic. In $\S 3$ we reproduce some number theory which came out of conversations with $\mathrm{S}$. Sperber. In $\S 4$ we introduce the classes $\zeta_{2 j}$ and $\xi_{2 j+1}$ and establish a number of relations among them. The classes $\zeta_{2 j}$ have been constructed as Chern classes of suitable complex representations in our paper [27]. In the present paper, we construct the classes $\xi_{2 j+1}$ by means of the transfer and the number theory in $\S 3$. In $\S 5$ we exploit a suitable homological perturbation to compute the characteristic classes introduced in our paper [26]. In general, these classes yield a description of the first nonvanishing differential of the spectral sequence of a group extension or, more generally, of the change of rings spectral sequence. In $\S 6$ of the present paper we exploit the calculations of the characteristic classes and, furthermore, the machinery in our earlier paper [26], thereby obtaining a proof of the following.

Theorem 0.4. In the integral cohomology spectral sequence $\left(E_{i}^{*, *}(\mathbf{e}, \mathbf{Z}), d_{i}\right)$ of the group extension e the first (possibly) nontrivial differential

$$
d_{3}: E_{3}^{k, 2 j} \longrightarrow E_{3}^{k+3,2 j-2}, \quad k \geq 0, j \geq 1,
$$

is zero for $k=2 i$, while for $k=2 i+1$,

$$
d_{3}: E_{3}^{2 i+1,2 j} \cong \mathbf{Z} / q_{j} \longrightarrow \mathbf{Z} / q_{j-1} \cong E_{3}^{2 i+4,2 j-2}, \quad i \geq 0, j \geq 1,
$$

sends an appropriate generator to $-\nu \frac{s}{(s, r)} \frac{q_{j-1}}{q_{1}}$ times an appropriate generator.

We note that for degree reasons the differential $d_{2}$ is zero and hence $d_{3}$ is the first possibly nonzero differential. The calculation of the latter relies on the number theory in $\S 3$. For example, the exact $p$-divisibility of the expression

$$
A(j, s, t)=j\left(1+t^{j-1}+\cdots+t^{(s-1)(j-1)}\right)-(j-1)\left(1+t^{j}+\cdots+t^{(s-1) j}\right)
$$

comes into play.

There is a notion of characteristic classes for group extensions due to Charlap and Vasquez [10,11]. These classes are defined only under strong additional hypotheses, and they assume their values in suitable group cohomology groups. Theorem 0.4 above shows that our notion of characteristic class really goes beyond what is in the literature. For the differential $d_{3}$ is nonzero if and only if $\nu \frac{s}{(s, r)}$ is not divisible by $q_{1}$. However, when the order $s$ of $K$ is odd, the cup pairing

$$
\cup: \mathrm{H}^{2 i+1}\left(K, \mathrm{H}^{2 j} N\right) \otimes \mathrm{H}^{3}\left(K, \operatorname{Hom}\left(\mathrm{H}^{2 j} N, \mathrm{H}^{2 j-2} N\right)\right) \longrightarrow \mathrm{H}^{2 i+4}\left(K, \mathrm{H}^{2 j-2} N\right)
$$

is zero for degree reasons, and so it is impossible to obtain the differential

$$
d_{3}: E_{3}^{2 i+1,2 j} \longrightarrow E_{3}^{2 i+4,2 j-2}
$$

by means of a characteristic class attaining its value in a group cohomology group. 
In $\S 6$ we prove Theorem 0.4; we then give the structure of $E_{4}$ in (6.9). Furthermore, a comparison of the subalgebra of the cohomology algebra generated by $\zeta_{2 j}$ and $\xi_{2 j+1}$ with $E_{4}$ enables us to conclude that, at a good prime $p$, the spectral sequence collapses from $E_{4}$ and that the cohomology is as an algebra generated by the classes $\zeta_{2 j}$ and $\xi_{2 j+1}$, in fact by a suitable initial finite subset thereof; details will be given in (6.11)-(6.13). In $\S 7$ we analyze the restriction of the spectral sequence to a suitable subgroup. In $\S 8$ we exploit the description of this restriction to see by induction that the relations among our classes given in $(0.3)$ are indeed defining relations, and in this way the usual additive extension problem is overcome. This will then complete the proof of Theorem 0.3 , our main result.

When $r_{p}<s_{p}$, at the prime $p$ the spectral sequence will not collapse from $E_{4}$ unless $f$ is divisible by $r_{p}$; if the latter happens to be true, the $p$-Sylow subgroup splits, and this case has been settled before. Finally, when $t-1$ is divisible by $f_{p}$, the presentation can be rewritten so that our approach is still applicable. Indeed, we may assume that $G$ is a $p$-group and that the numbers $r, s$, and $f$ are $p$ th powers. We can then project $G$ onto $\mathbf{Z} /(t-1)_{p}$ by sending $y$ to a generator and $x$ to zero. The kernel of this projection is generated by $x$ and $y^{t-1}$. When $t-1$ is divisible by $f=f_{p}$,

$$
y^{t-1}=\left(y^{f}\right)^{\frac{t-1}{f}}=x^{s \frac{t-1}{f}}
$$

the kernel is cyclic, and we are in the situation of Theorem 0.3.

In the present paper we do not give the multiplicative structure apart from the structure as a module over the cohomology of the quotient group $K$. We intend to determine the ring structure elsewhere.

The integral homology of a split metacyclic group has been given by Wall [46], and it is straightforward to dualize his description to obtain the additive structure of the integral cohomology of such a group (cf. Thomas [44]). The integral cohomology ring of a (necessarily split) metacyclic $p$-group of order $p^{3}$ for $p$ odd has been given by Lewis [38]; more generally, Larson [36] computed the integral cohomology ring of split metacyclic groups $G$ which are extensions of the form

$$
0 \rightarrow \mathbf{Z} / p^{n} k \rightarrow G \rightarrow \mathbf{Z} / p \rightarrow 1,
$$

where $p$ is a prime and $k$ is prime to $p$. The cohomology of the dihedral group and quaternion group is classical (cf., e.g., Madsen-Milgram [40] and Cartan-Eilenberg [8]). Further, Evens and Priddy [12] computed the integral cohomology of semidihedral groups. In [1] Alzubaidy claims to have computed the integral cohomology of any metacyclic $p$-group but a brief look at Wall's results [46] shows that Alzubaidy's results cannot be correct; also it was pointed out to me by Yagita that Alzubaidy's results are inconsistent with what is said in Tezuka and Yagita [42].

Much of this work was done during a stay at the Institute for Advanced Study. I should like to express my gratitude for its hospitality. I am indebted to 
S. Sperber for conversations about number theory and to the referee for some comments which helped improve the exposition.

\section{Perturbations and Wall resolutions}

In this section we use "homological perturbation theory" to elaborate on a resolution whose existence was first proved by Wall [46]; this resolution also occurs in Lazard [37]. The interested reader may find more about homological perturbation theory in [7, 9, 13-21, 24, 28, 30-35]. We shall feel free to use the same material, notation, results, etc. as in $\S 2$ of [30], and we shall not repeat it. As in the Introduction, $G$ will be a metacyclic group, given by a presentation (0.1), and we shall maintain the notation in the Introduction.

Let $R$ be a commutative ring with 1 , taken henceforth as ground ring. We denote the divided polynomial algebra on a single generator $u$ by $\Gamma[u]$ and the exterior algebra on a single generator $v$ by $\Lambda[v]$. With this notation, the standard small free resolutions for the kernel $N$ and quotient $K$ of e may be written

$$
\begin{aligned}
& \mathbf{M}(N)=\left(\Gamma\left[u_{y}\right] \otimes \Lambda\left[v_{y}\right] \otimes R N, d\right)=\mathbf{M}^{\sharp}(N) \otimes_{d} R N, \\
& \mathbf{M}(K)=\left(\Gamma\left[u_{x}\right] \otimes \Lambda\left[v_{x}\right] \otimes R K, d\right)=\mathbf{M}^{\sharp}(K) \otimes_{d} R K,
\end{aligned}
$$

where $\mathbf{M}^{\sharp}(N)=\Gamma\left[u_{y}\right] \otimes \Lambda\left[v_{y}\right]$ and $\mathbf{M}^{\sharp}(K)=\Gamma\left[u_{x}\right] \otimes \Lambda\left[v_{x}\right]$; details may be found e.g., on p. 252 of Cartan-Eilenberg [8], where the resolution is written $X_{L}$ and where the name divided polynomial algebra does not occur but its structure is given explicitly. As in [30] we write a Wall resolution [46] of $R$ in the category of right $R G$-modules in the form

$$
\mathbf{M}(G)=\left(\mathbf{M}^{\sharp}(K) \otimes \mathbf{M}^{\sharp}(N) \otimes R G, d\right), \quad \text { with } \quad d=d^{0}+d^{1}+d^{2}+\cdots,
$$

so that

$$
d^{0}=\mathrm{M}^{\sharp}(K) \otimes d_{\mathrm{M}^{\sharp}(N) \otimes_{d} R G} \quad\left(=\mathrm{Id}_{\mathrm{M}^{\sharp}(K)} \otimes d_{\mathrm{M}^{\sharp}(N) \otimes_{d} R G}\right),
$$

and so that, for $i \geq 1$, the operator $d^{i}$ lowers Serre filtration (i.e., the filtration by $\left(\mathbf{M}^{\sharp}(K)\right)$-degree) by $i$; for completeness we mention that $d_{\mathrm{M}^{\sharp}(N) \otimes_{d} R G}$ refers to the differential on $\mathrm{M}(N) \otimes_{R N} R G$ induced by the one on $\mathrm{M}(N)$. The operator $d^{0}$ is an $R G$-linear differential on $\mathbf{M}^{\sharp}(K) \otimes \mathbf{M}^{\sharp}(N) \otimes R G$; it is vertical in an obvious sense. It has become customary (see, e.g., [17, 24, 25, 30, 33-35]) to refer to the infinite series $\partial=d^{1}+d^{2}+\cdots$ as a perturbation of $d^{0}$; see also Remark 1.5 below for more comments about perturbation theory.

For intelligibility we recall that the property $d d=0$ of the differential $d=$ $d^{0}+\partial$ amounts to the sequence

$$
d^{0} d^{i}+d^{1} d^{i-1}+\cdots+d^{i} d^{0}=0, \quad i \geq 0,
$$

of indicated identities. Moreover, once the perturbation $\partial$ has been obtained, the obvious projection map is a morphism

$$
g: \mathbf{M}(G)=\left(\mathbf{M}^{\sharp}(K) \otimes \mathbf{M}^{\sharp}(N) \otimes R G, d\right) \longrightarrow \mathbf{M}(K)=\left(\mathbf{M}^{\sharp}(K) \otimes R K, d\right)
$$


of filtered chain complexes and is compatible with the obvious right $R G$-module structures. A standard spectral sequence comparison argument then shows that the spectral sequence $\left(E_{*}^{*, *}(\mathbf{M}(G)), d_{*}\right)$ coming from the Serre filtration has $\left(E_{1}, d_{1}\right)=\mathbf{M}(K)$. Consequently

$$
\mathbf{M}(G)=\left(\mathbf{M}^{\sharp}(K) \otimes \mathbf{M}^{\sharp}(N) \otimes R G, d\right)
$$

is acyclic and hence its underlying chain complex is a free resolution of $R$ in the category of right $R G$-modules.

Suitable terms $d^{1}$ and $d^{2}$ for the split case (i.e., the case where $f=0$ ) have been given in Wall [46], and, as observed in [46], $d^{0}+d^{1}+d^{2}$ is then a differential on $\mathrm{M}(G)$. In general it is difficult to find explicit formulas for the higher terms of the perturbation. For our purposes it will suffice to have available only some of them. We now give the requisite information; here and henceforth we shall write, for conciseness,

$$
x_{k}=1+x+\cdots+x^{k-1}, \quad y_{l}=1+y+\cdots+y^{l-1}, \quad \text { etc. }
$$

By construction, we have

$$
\begin{aligned}
d^{0}\left(\gamma_{\mu}\left(u_{x}\right) \gamma_{j}\left(u_{y}\right)\right) & =\left(\gamma_{\mu}\left(u_{x}\right)\left(\gamma_{j-1}\left(u_{y}\right)\right) v_{y}\right) y_{r}, \quad j \geq 1, \\
d^{0}\left(\gamma_{\mu}\left(u_{x}\right)\left(\gamma_{j}\left(u_{y}\right)\right) v_{y}\right) & =\left(\gamma_{\mu}\left(u_{x}\right) \gamma_{j}\left(u_{y}\right)\right)(y-1), \quad j \geq 0, \\
d^{0}\left(\gamma_{\mu}\left(u_{x}\right) v_{x}\left(\gamma_{j}\left(u_{y}\right)\right)\right) & =-\gamma_{\mu}\left(u_{x}\right) v_{x}\left(\left(\gamma_{j-1}\left(u_{y}\right)\right) v_{y}\right) y_{r}, \quad j \geq 0, \\
d^{0}\left(\gamma_{\mu}\left(u_{x}\right) v_{x}\left(\gamma_{j}\left(u_{y}\right)\right) v_{y}\right) & =-\gamma_{\mu}\left(u_{x}\right) v_{x}\left(\gamma_{j}\left(u_{y}\right)\right)(y-1), \quad j \geq 0 .
\end{aligned}
$$

Let

$$
\begin{aligned}
d^{1}\left(\gamma_{\mu}\left(u_{x}\right) \gamma_{j}\left(u_{y}\right)\right) & =\gamma_{\mu-1}\left(u_{x}\right) v_{x} \gamma_{j}\left(u_{y}\right)\left(1+\left(\left(y_{t}\right)^{j} x\right)+\cdots+\left(\left(y_{t}\right)^{j} x\right)^{s-1}\right), \\
d^{1}\left(\gamma_{\mu}\left(u_{x}\right) \gamma_{j}\left(u_{y}\right) v_{y}\right)= & \gamma_{\mu-1}\left(u_{x}\right) v_{x} \gamma_{j}\left(u_{y}\right) v_{y} \\
& \times\left(1+\left(\left(y_{t}\right)^{j+1} x\right)+\cdots+\left(\left(y_{t}\right)^{j+1} x\right)^{s-1}\right), \\
d^{1}\left(\gamma_{\mu}\left(u_{x}\right) v_{x} \gamma_{j}\left(u_{y}\right)\right)= & \gamma_{\mu}\left(u_{x}\right) \gamma_{j}\left(u_{y}\right)\left(\left(y_{t}\right)^{j} x-1\right), \\
d^{1}\left(\gamma_{\mu}\left(u_{x}\right) v_{x} \gamma_{j}\left(u_{y}\right) v_{y}\right)= & \gamma_{\mu}\left(u_{x}\right) \gamma_{j}\left(u_{y}\right) v_{y}\left(\left(y_{t}\right)^{j+1} x-1\right), \\
d^{2}\left(\gamma_{\mu}\left(u_{x}\right) \gamma_{j}\left(u_{y}\right)\right) & =-\gamma_{\mu-1}\left(u_{x}\right) \gamma_{j}\left(u_{y}\right) v_{y} y_{f}, \\
d^{2}\left(\gamma_{\mu}\left(u_{x}\right) \gamma_{j}\left(u_{y}\right) v_{y}\right) & =-\frac{t^{(j+1) s}-1}{r} \gamma_{\mu-1}\left(u_{x}\right) \gamma_{j+1}\left(u_{y}\right), \\
d^{2}\left(\gamma_{\mu}\left(u_{x}\right) v_{x} \gamma_{j}\left(u_{y}\right)\right) & =\gamma_{\mu-1}\left(u_{x}\right) v_{x} \gamma_{j}\left(u_{y}\right) v_{y} y_{f}, \\
d^{2}\left(\gamma_{\mu}\left(u_{x}\right) v_{x} \gamma_{j}\left(u_{y}\right) v_{y}\right)= & \frac{t^{(j+1) s}-1}{r} \gamma_{\mu-1}\left(u_{x}\right) v_{x} \gamma_{j+1}\left(u_{y}\right), \\
d^{3}\left(\gamma_{\mu}\left(u_{x}\right) v_{x} \gamma_{j}\left(u_{y}\right)\right) & =-(j+1) \frac{(t-1) f}{r} \gamma_{\mu-1}\left(u_{x}\right) \gamma_{j+1}\left(u_{y}\right) y^{f}\left(y_{t}\right)^{j} x, \\
d^{3}\left(\gamma_{\mu}\left(u_{x}\right) v_{x} \gamma_{j}\left(u_{y}\right) v_{y}\right) & =-(j+1) \frac{(t-1) f}{r} \gamma_{\mu-1}\left(u_{x}\right) \gamma_{j+1}\left(u_{y}\right) v_{y} y^{f}\left(y_{t}\right)^{j+1} x .
\end{aligned}
$$


It is then straightforward to check that the identity (1.0.i) holds whenever it is already defined. By Lemma 2.10 of [30], the construction of the perturbation can always be completed, and we obtain a differential $d$ on $\mathbf{M}(G)$ as desired. We shall not need formulas for the remaining values of the operator $d^{3}$, nor shall we need formulas for the higher terms of the perturbation. The above formulas for the operators $d^{1}, d^{2}, d^{3}$ have been obtained by means of an appropriate perturbation theory. Space does permit us to give the details here and we shall explain them elsewhere [28].

Remark 1.3. Inspection shows that, when $f=0$ so that the extension e splits, $d^{0}+d^{1}+d^{2}$ is a differential on $\mathrm{M}(G)$. We have already pointed out that this observation is due to Wall [46]. Likewise, when $t=1, G$ is abelian, and again inspection shows that $d^{0}+d^{1}+d^{2}$ is a differential on $\mathrm{M}(G)$.

Remark 1.4. In the general case, let $\eta: R \longrightarrow \mathrm{M}(N)$ be the obvious map, choose a section $\sigma: K \longrightarrow G$ for the projection $\pi: G \rightarrow K$ of the underlying sets so that $\sigma(1)=1$ and $\pi \sigma=\operatorname{Id}_{K}$, write $\sigma^{b}: R K \longrightarrow R G$ for the induced morphism of $R$-modules, and let

$$
\nabla_{\sigma}=\operatorname{Id}_{M^{\sharp}(K)} \otimes \eta \otimes \sigma^{b}: \mathrm{M}^{\sharp}(K) \otimes R K \longrightarrow\left(\mathrm{M}^{\sharp}(K) \otimes \mathrm{M}^{\sharp}(N)\right) \otimes R G .
$$

This is a section of $g$ for the underlying graded $R$-modules. Moreover, let $s_{N}$ be a contracting homotopy of $\mathrm{M}(N)$ so that

$$
d s_{N}+s_{N} d=\eta \varepsilon-\mathrm{Id}_{\mathrm{M}(N)},
$$

and define a morphism

$$
h:\left(\mathbf{M}^{\sharp}(K) \otimes \mathbf{M}^{\sharp}(N)\right) \otimes R G \longrightarrow\left(\mathbf{M}^{\sharp}(K) \otimes \mathbf{M}^{\sharp}(N)\right) \otimes R G
$$

of graded modules of degree 1 by means of the formula

$$
h(b \otimes a \otimes w)=(-1)^{|b|}\left(b \otimes s_{N}\left(a \otimes w(\sigma \pi(w))^{-1}\right)\right)(\sigma \pi(w)),
$$

where $b \in \mathbf{M}^{\sharp}(K), a \in \mathbf{M}^{\sharp}(N), w \in G$. Then

$$
d^{0} h+h d^{0}=\nabla_{\sigma} g-\text { Id }, \quad g h=0 .
$$

If, in addition, $s_{N} \eta=0$ and $s_{N}$ has square zero, we also have

$$
h \nabla_{\sigma}=0, \quad h h=0 ;
$$

since the underlying graded abelian group of $\mathrm{M}(N)$ is free abelian, such an $s_{N}$ exists, as is well known. The data

$$
(M(G) \stackrel{g}{\stackrel{g}{\rightleftarrows}} \mathrm{M}(K), h)
$$

then constitute an $R G$-module contraction in the sense of [24] (see also $\S 2$ of [30]). In particular, when we combine $h$ with a contracting homotopy for $\mathrm{M}(K)$ we obtain a contracting homotopy for $\mathrm{M}(G)$, and we see once again that $\mathrm{M}(G)$ is acyclic. 
Remark 1.5. The problem we are faced with in the present section is that of constructing a differential on the "bigger" object $\left(\mathbf{M}^{\sharp}(K) \otimes \mathbf{M}^{\sharp}(N)\right) \otimes R G$ (where $M(K)$ is the "smaller" one). A solution of this problem (or a partial one as above) could hence be referred to as a blowing up construction; blowing up constructions occur also in [18,20,24, 28, 30, 32, 33, 34]. The papers [13-17, $19,21,35$ ] deal with a different kind of problem which might be called a blowing down construction (see, in particular, [17]); to our knowledge, such a blowing down construction occurs first in [7]. It is also used in [9, 24, 31, 33, 34].

\section{THE COHOMOLOGY IN A NUMBER OF SPECIAL CASES}

As before let $G$ be a metacyclic group, given by a presentation (0.1), with corresponding group extension e (cf. $(0.2))$. In the present section we give a description of the additive structure of the cohomology of $G$ in a number of special cases. Later in the paper we shall reduce the calculation of cohomology in the general case to one of these special cases.

For completeness, we begin with a split metacyclic group $G$; this is one so that the extension e splits. In this case, after having rechosen the generator $x$ suitably (if necessary) we may assume that $f=0$, and we shall henceforth do so. We have already observed that the formulas (1.1) and (1.2) then yield a differential $d=d^{0}+d^{1}+d^{2}$ on $\mathrm{M}(G)$. We now consider the reduced dual object

$$
\overline{\mathbf{M}}(G)^{*}=\operatorname{Hom}_{R G}(\mathbf{M}(G), R)=\operatorname{Hom}\left(\mathbf{M}(G) \otimes_{R G} R, R\right) .
$$

By construction, as an induced $\mathrm{P}\left[c_{x}\right]$-module, $\overline{\mathrm{M}}(G)^{*}$ may be written

$$
\overline{\mathbf{M}}(G)^{*}=\mathrm{P}\left[c_{x}\right] \otimes \Lambda\left[\omega_{x}\right] \otimes \mathrm{P}\left[c_{y}\right] \otimes \Lambda\left[\omega_{y}\right] .
$$

To describe its differential explicitly, we write $\omega_{x}, \omega_{y}, c_{x}, c_{y}$ for the duals in the graded sense of, respectively, $v_{x}, v_{y}, u_{x}, u_{y}$ in the basis of monomials. Henceforth we use the Eilenberg-Koszul convention to the effect that, for example, $\left(\omega_{x} \omega_{y}\right)\left(v_{x} v_{y}\right)=-1$, and that the coboundary $d c$ of a cochain $c$ is given by the formula $d(c)=(-1)^{|c|+1} c d$. With this convention, as morphisms of graded $\mathrm{P}\left[c_{x}\right]$-modules, the operators $d_{0}, d_{1}, d_{2}$ are given by the formulas indicated in the following table:

$$
\begin{array}{cccc} 
& d_{0} & d_{1} & d_{2} \\
c_{y}^{j} & 0 & -\left(t^{j}-1\right) \omega_{x} c_{y}^{j} & +\frac{t^{j s}-1}{r} c_{x} c_{y}^{j-1} \omega_{y} \\
c_{y}^{j} \omega_{y} & r c_{y}^{j+1} & -\left(t^{j+1}-1\right) \omega_{x} c_{y}^{j} \omega_{y} & 0 \\
\omega_{x} c_{y}^{j} & 0 & +\left(1+\left(t^{j}\right)+\cdots+\left(t^{j}\right)^{s-1}\right) c_{x} c_{y}^{j} & -\frac{t^{j s}-1}{r} c_{x} \omega_{x} c_{y}^{j-1} \omega_{y} \\
\omega_{x} c_{y}^{j} \omega_{y} & -r \omega_{x} c_{y}^{j+1} & +\left(1+\left(t^{j+1}\right)+\cdots+\left(t^{j+1}\right)^{s-1}\right) c_{x} c_{y}^{j} \omega_{y} & 0
\end{array}
$$

It is clear that $\overline{\mathbf{M}}(G)^{*}$ is filtered by $\left(\mathbf{M}^{\sharp}(K)\right)$-degree, and that the resulting spectral sequence is just the spectral sequence $\left(E_{i}^{*, *}(\mathbf{e}, R), d_{i}\right)$ of the group extension e. We now take $R$ to be the integers $Z$. In order to write down the graded group $E_{2}$ we remind the reader that, for $j \geq 0$, the numbers $h_{j}, k_{j}, q_{j}$ 
have been defined in the Introduction. With this notation it is clear that the integral cohomology spectral sequence has

$$
\begin{array}{llll}
E_{2}^{0,2 j}=\frac{r}{h_{j}} c_{y}^{j} \mathbf{Z} / r & \cong \mathbf{Z} / h_{j}, & j \geq 1 ; \\
E_{2}^{2 i, 2 j}=\frac{r}{h_{j}} c_{x}^{i} c_{y}^{j} \mathbf{Z} / k_{j} & \cong \mathbf{Z} / q_{j}, & i \geq 1, j \geq 0 ; \\
E_{2}^{2 i, 2 j+1}=0=E_{2}^{2 i+1,0}=E_{2}^{2 i+1,2 j+1}, & & i \geq 0, j \geq 0 ; \\
E_{2}^{2 i-1,2 j}=\frac{r}{k_{j}} c_{x}^{i-1} \omega_{x} c_{y}^{j} \mathbf{Z} / h_{j} & \cong \mathbf{Z} / q_{j}, & i \geq 1, j \geq 1 .
\end{array}
$$

For later reference we record that, with the notation adjusted appropriately, this description of $E_{2}$ is valid for any extension of the kind (0.2), that is, for any extension of a finite cyclic group by a finite cyclic group. Moreover, in view of what was said above about the differential in $\overline{\mathrm{M}}(G)^{*}$, in the split case the spectral sequence has $E_{2}=E_{\infty}$. The graded group $E_{2}$ can of course be computed directly as the cohomology of the cyclic group $K$ with respect to the induced action on $\mathrm{H}^{*}(N, \mathbf{Z})$.

Still examining the split case, for $j \geq 1$, we now consider the following cochains, where subscripts indicate degrees:

$$
\begin{aligned}
\zeta_{2 j} & =\frac{r}{h_{j}} c_{y}^{j}-\frac{t^{j}-1}{h_{j}} \omega_{x} c_{y}^{j-1} \omega_{y}, \\
\xi_{2 j+1} & =\frac{r}{k_{j}} \omega_{x} c_{y}^{j}-\frac{1+\left(t^{j}\right)+\cdots+\left(t^{j}\right)^{s-1}}{k_{j}} c_{x} c_{y}^{j-1} \omega_{y} .
\end{aligned}
$$

Inspection shows that

$$
\begin{gathered}
\left(d_{0}+d_{1}+d_{2}\right)\left(c_{y}^{j-1} \omega_{y}\right)=h_{j} \zeta_{2 j}, \\
\left(d_{0}+d_{1}+d_{2}\right)\left(c_{y}^{j}\right)=-\frac{\left(t^{j}-1\right) k_{j}}{r} \xi_{2 j+1}, \\
\left(d_{0}+d_{1}+d_{2}\right)\left(\omega_{x} c_{y}^{j-1} \omega_{y}\right)=-k_{j} \xi_{2 j+1}, \\
\left(d_{0}+d_{1}+d_{2}\right)\left(\omega_{x} c_{y}^{j}\right)=\frac{\left(1+\left(t^{j}\right)+\cdots+\left(t^{j}\right)^{s-1}\right) h_{j}}{r} c_{x} \zeta_{2 j} .
\end{gathered}
$$

The additive structure of the homology of a split metacyclic group is due to Wall [46] and has been known for a while, and the additive structure of the cohomology of such a group is obtained from that of its homology in the standard way. We therefore label the following result as a proposition.

Proposition 2.8. In the split case the cochains $\zeta_{2 j}$ and $\xi_{2 j+1}$ are cocycles, and as a graded module over the cohomology ring $\mathrm{H}^{*}(\mathbf{Z} / s, \mathbf{Z})$ of the quotient group $\mathbf{Z} / s$, the integral cohomology $\mathrm{H}^{*}(G, \mathbf{Z})$ is generated by (the classes of) $\zeta_{2 j}$ and 
$\xi_{2 j+1}$, subject to the relations

$$
\begin{aligned}
h_{j} \zeta_{2 j} & =0, \\
q_{j} c_{x} \zeta_{2 j} & =0, \\
q_{j} \xi_{2 j+1} & =0 .
\end{aligned}
$$

In particular, there is no additive extension problem from $E_{\infty}=E_{2}$ to $\mathrm{H}^{*}(G, \mathbf{Z})$. Finally, as a ring, the integral cohomology $\mathrm{H}^{*}(G, \mathbf{Z})$ is generated by (the classes of) $\zeta_{2 j}$ and $\xi_{2 j+1}$ for $j \leq s$.

Proof. It is clear that $\zeta_{2 j}$ and $\xi_{2 j+1}$ are cocycles, and (2.4)-(2.7) imply that the relations (2.9)-(2.11) hold. A brief look at the description of $E_{2}$ of the spectral sequence given above shows at once that these are defining relations and that there is in fact no additive extension problem. Finally, the multiplicative structure of the spectral sequence implies that, as a ring, the integral cohomology $\mathrm{H}^{*}(G, \mathbf{Z})$ is generated by (the classes of) $\zeta_{2 j}$ and $\xi_{2 j+1}$ for $j \leq s$ as asserted.

Remark 2.12. The argument given by Wall [46] for the computation of the homology of $G$ avoids the explicit construction of cycles. Here is its counterpart for cohomology: Inspection shows that the object $\overline{\mathrm{M}}(G)^{*}$ decomposes as a direct sum

$$
\overline{\mathbf{M}}(G)^{*}=\left(\mathrm{P}\left[c_{x}\right] \otimes \Lambda\left[\omega_{x}\right], d\right) \oplus \bigoplus_{j \geq 1}\left(\mathrm{P}\left[c_{x}\right] \otimes \Lambda\left[\omega_{x}\right] \otimes\left(\mathbf{Z}\left\langle c_{y}^{j-1} \omega_{y}\right\rangle \oplus \mathbf{Z}\left\langle c_{y}^{j}\right\rangle\right), d\right)
$$

of filtered chain complexes. Since in each dimension the homology of each summand is at most a cyclic group, there is no additive extension problem for each summand, whence there is no additive extension problem from $E_{\infty}=E_{2}$ to $\mathrm{H}^{*}(G, \mathbf{Z})$.

Let now $G$ be an arbitrary metacyclic group, given by a presentation $(0.1)$. Our next aim is to compute the cohomology of $G$ at the primes $p$ so that the restriction of e to the $p$-Sylow subgroup of $K$ splits; under such circumstances we shall occasionally say that e splits at $p$.

Let $p$ be a prime dividing $r$ and $s$. We shall write

$$
\mathbf{e}^{p}: 1 \longrightarrow N \longrightarrow G_{p} \longrightarrow K_{p} \longrightarrow 1
$$

for the restriction of the extension e to the $p$-primary part $K_{p}$ of $K$, and we shall denote by

$$
\tau_{G_{p} \uparrow G}: \mathrm{H}^{*}\left(G_{p}, \mathbf{Z}\right) \longrightarrow \mathrm{H}^{*}(G, \mathbf{Z})
$$

the corresponding transfer. If the extension $\mathrm{e}^{p}$ splits we denote, for $j \geq 1$, the generators of $\mathrm{H}^{*}\left(G_{p}, \mathbf{Z}\right)$ given in $(2.7)$ above by $\zeta_{2 j}^{\prime} \in \mathrm{H}^{2 j}\left(G_{p}, \mathbf{Z}\right)$ and $\xi_{2 j+1}^{\prime} \in \mathrm{H}^{2 j+1}\left(G_{p}, \mathbf{Z}\right)$; under such circumstances we shall write

$$
\zeta_{2 j}=\tau_{G_{p} \uparrow G}\left(\zeta_{2 j}^{\prime}\right) \in \mathrm{H}^{2 j}(G, \mathbf{Z}), \quad \xi_{2 j+1}=\tau_{G_{p} \uparrow G}\left(\xi_{2 j+1}^{\prime}\right) \in \mathrm{H}^{2 j+1}(G, \mathbf{Z}) .
$$


We now phrase the property that the extension $\mathrm{e}^{p}$ splits in terms of the numerical parameters $r, s, t, f$. To this end, we consider the second cohomology group $\mathrm{H}^{2}(K, N)$ of $K=\left\langle x ; x^{s}\right\rangle \cong \mathbf{Z} / s$ with coefficients in $N=\left\langle y ; y^{r}\right\rangle \cong \mathbf{Z} / r$ with respect to the action of $K$ on $N$ coming from conjugation in $G$. Since this action is given by ${ }^{x} y=y^{t}$, it is clear that $\mathrm{H}^{2}(K, N)$ is the homology of

$$
\mathbf{Z} / r \stackrel{1+t+\cdots+t^{s-1}}{\longrightarrow} \mathbf{Z} / r \stackrel{t-1}{\longrightarrow} \mathbf{Z} / r,
$$

whence

$$
\begin{aligned}
\mathrm{H}^{2 \cdot}(K, N) & =\left(\frac{r}{(t-1, r)} \mathbf{Z} / r\right) /\left(1+t+\cdots+t^{s-1}\right) \\
& \cong \mathbf{Z} /\left(\frac{(t-1, r)\left(1+t+\cdots+t^{s-1}, r\right)}{r}\right)=\mathbf{Z} / q_{1} .
\end{aligned}
$$

Since $t f \equiv f \bmod r$, the number $f$ may be written $f=\nu \frac{r}{(t-1, r)}$, and $\nu$ modulo $q_{1}$ is precisely the class of the extension e in $\mathrm{H}^{2}(K, N)$. Notice in particular that, as far as the parameter $f$ is concerned, in the above presentation it suffices to restrict attention to the numbers $\nu$ so that $\nu$ divides $q_{1}=\frac{(t-1, r)\left(1+t+\cdots+t^{s-1}, r\right)}{r}$. For these matters, see also IV.2.8 of Beyl-Tappe [5].

For a prime $p$ and an arbitrary number $m$ we shall henceforth denote the $p$-primary part of $m$ by $m_{p}$ so that $m=m_{p} m^{\prime}$ with $m^{\prime}$ prime to $p$.

Theorem 2.15. Let $G=G(r, s, t, f)$ be an arbitrary metacyclic group with corresponding group extension $\mathbf{e}$, and let $p$ be a prime so that $\nu \equiv 0 \bmod \left(q_{1}\right)_{p}$. Then at $p$ the homology and cohomology spectral sequences collapse from $E^{2}$ and $E_{2}$ respectively, and there is no additive extension problem from $E_{\infty}=E_{2}$ to the p-primary part $\mathrm{H}^{*}(G, \mathbf{Z})_{p}$ of $\mathrm{H}^{*}(G, \mathbf{Z})$. Furthermore, as a graded module over the cohomology ring $\mathrm{H}^{*}(\mathbf{Z} / s, \mathbf{Z})$ of the quotient group $\mathbf{Z} / s$, at $p$ the integral cohomology $\mathrm{H}^{*}(G, Z)$ is generated by $\zeta_{2 j}$ and $\xi_{2 j+1}$, subject to the relations

$$
\begin{aligned}
h_{j} \zeta_{2 j} & =0, \\
q_{j} c_{x} \zeta_{2 j} & =0, \\
q_{j} \xi_{2 j+1} & =0 .
\end{aligned}
$$

Proof. It is clear that $\nu \equiv 0 \bmod \left(q_{1}\right)_{p}$ if and only if the restriction map into $\mathrm{H}^{2}\left(K_{p}, N\right)$ is zero, i.e., if and only if the extension $\mathrm{e}^{p}$ splits. By Proposition 2.8 the cohomology spectral sequence $\left(E_{i}^{*, *}\left(\mathbf{e}^{p}, \mathbf{Z}\right), d_{i}\right)$ of the latter collapses from $E_{2}$. Since the index of $K_{p}$ in $K$ is prime to $p$, the standard argument shows that the restriction map

$$
\mathrm{H}^{*}\left(K, \mathrm{H}^{*}(N, \mathbf{Z})\right)_{p} \longrightarrow \mathrm{H}^{*}\left(K_{p}, \mathrm{H}^{*}(N, \mathbf{Z})\right)
$$

is injective. Hence at $p$ higher differentials in the spectral sequence of $\mathbf{e}$ are detected in the spectral sequence of $\mathrm{e}^{p}$ which has no higher differentials. Consequently at $p$ the spectral sequence $\left(E_{i}^{*, *}(\mathbf{e}, \mathbf{Z}), d_{i}\right)$ has $E_{2}=E_{\infty}$. 
To complete the proof, let $j_{0}$ be the order of $t$ modulo $p$, i.e., the smallest integer $j$ so that $t^{j} \equiv 1 \bmod p$. It is clear that the numbers $h_{j}$ and $q_{j}$ are prime to $p$ unless $j$ is a multiple of $j_{0}$. Hence at $p$ the group $E_{\infty}^{*, 2 j}(\mathbf{e}, \mathbf{Z})$ is nonzero only if $j$ is a multiple of $j_{0}$. Further, it is clear that at $p$ the transfer

$$
\tau_{K_{p} \uparrow K}: E_{\infty}^{*, 2 j}\left(\mathbf{e}^{p}, \mathbf{Z}\right) \longrightarrow E_{\infty}^{*, 2 j}(\mathbf{e}, \mathbf{Z})
$$

is trivial if $j$ is not divisible by $j_{0}$, and a little thought reveals that at $p$ this transfer is an isomorphism if $j$ is divisible by $j_{0}$. It follows that, as a graded module over the cohomology ring $\mathrm{H}^{*}(\mathbf{Z} / s, \mathbf{Z})$ of the quotient group $\mathbf{Z} / s$, at $p$ the integral cohomology $\mathrm{H}^{*}(G, \mathbf{Z})$ has the asserted structure. Notice that the relations $(2.16)-(2.18)$ have real content only if $j$ is divisible by $j_{0}$.

Remark 2.19. It is clear that for any prime $p$ relatively prime to the order $q_{1}$ of $\mathrm{H}^{2}(K, N)$ the hypothesis of the theorem is satisfied, whatever $\nu$.

Remark 2.20. It is also clear that, with a notation introduced in (2.14), an arbitrary metacyclic group $G$ fits into an extension

$$
1 \longrightarrow G_{p} \longrightarrow G \longrightarrow K / K_{p} \longrightarrow 1,
$$

where the order of the quotient $K / K_{p}$ is prime to $p$. Hence at $p$ the cohomology of $G$ consists precisely of the invariants of the cohomology of $G_{p}$ under the induced action. The above argument yields among others an explicit calculation of these invariants in the case where the extension $\mathbf{e}^{p}$ splits.

Our next special case is that of an abelian metacyclic group $G$, given by a presentation (0.1) with $t=1$. We shall need an explicit description of the cohomology of $G$ in terms of the generators in $(0.1)$.

It is clear that, after having rechosen the generators $x$ and $y$ suitably (if necessary), we may assume that the number $f$ is nonzero and divides $r$ and $s$; in particular, in this description the extension e splits if and only if $f=(r, s)$. Then $G$ may be written as a direct product of two cyclic groups generated by $x^{\prime}=x$ and $y^{\prime}=x^{-\frac{s}{f}} y$ of order $s^{\prime}=\frac{r s}{f}$ and $f$ respectively; if $f=1$ the second factor is of course trivial but this is of no account for what will be said below. Following the recipe in Cartan-Eilenberg [8], we can compute from this the integral cohomology ring of $G$ as follows:

Using a notation explained in the previous subsection, over an arbitrary ground ring $R$ we may write the standard small free resolutions $\mathbf{M}\left(\mathbf{Z} / s^{\prime}\right)$ and $\mathbf{M}(\mathbf{Z} / f)$ for $\mathbf{Z} / s^{\prime}$ and $\mathbf{Z} / f$ in the form

$$
\mathbf{M}\left(\mathbf{Z} / s^{\prime}\right)=\mathbf{M}^{\sharp}\left(\mathbf{Z} / s^{\prime}\right) \otimes_{d} R\left(\mathbf{Z} / s^{\prime}\right), \quad \mathbf{M}(\mathbf{Z} / f)=\mathbf{M}^{\sharp}(\mathbf{Z} / f) \otimes_{d} R(\mathbf{Z} / f),
$$

respectively, where

$$
\mathbf{M}^{\sharp}\left(\mathbf{Z} / s^{\prime}\right)=\Gamma\left[u_{x^{\prime}}\right] \otimes \Lambda\left[v_{x^{\prime}}\right], \quad \mathbf{M}^{\sharp}(\mathbf{Z} / f)=\Gamma\left[u_{y^{\prime}}\right] \otimes \Lambda\left[v_{y^{\prime}}\right],
$$

and it is clear that their tensor product

$$
\mathbf{M}(G)=\mathbf{M}\left(\mathbf{Z} / s^{\prime}\right) \otimes \mathbf{M}(\mathbf{Z} / f)
$$


is a free resolution for $G$. Furthermore, it is well known that the diagonal map $\Delta$ given in Cartan-Eilenberg [8] turns $\mathbf{M}\left(\mathbf{Z} / s^{\prime}\right)$ and $\mathbf{M}(\mathbf{Z} / f)$ into (noncocommutative) differential graded coalgebras, and the tensor product structure turns $\mathrm{M}(G)$ into a differential graded coalgebra. This implies that, as a graded algebra, the reduced dual object

$$
\overline{\mathbf{M}}(G)^{*}=\operatorname{Hom}_{R G}(\mathbf{M}(G), R)=\operatorname{Hom}\left(\mathbf{M}(G) \otimes_{R G} R, R\right)
$$

is generated by the duals $\omega_{x^{\prime}}, \omega_{y^{\prime}}, c_{x^{\prime}}, c_{y^{\prime}}$ in the graded sense of, respectively, $v_{x^{\prime}}, v_{y^{\prime}}, u_{x^{\prime}}, u_{y^{\prime}}$ in the basis of monomials; moreover, the generators commute in the graded sense, save that the squares of the odd degree generators are nonzero and given by

$$
\omega_{x^{\prime}}^{2}=\frac{s^{\prime}\left(s^{\prime}-1\right)}{2} c_{x^{\prime}}, \quad \omega_{y^{\prime}}^{2}=\frac{f(f-1)}{2} c_{y^{\prime}}
$$

finally, the differential $d$ on $\overline{\mathrm{M}}(G)^{*}$ is given by

$$
d\left(\omega_{x^{\prime}}\right)=s^{\prime} c_{x^{\prime}}, \quad d\left(c_{x^{\prime}}\right)=0, \quad d\left(\omega_{y^{\prime}}\right)=f c_{y^{\prime}}, \quad d\left(c_{y^{\prime}}\right)=0
$$

Then it is clear that

$$
\xi_{3}=\frac{s^{\prime}}{f} c_{x^{\prime}} \omega_{y^{\prime}}-\omega_{x^{\prime}} c_{y^{\prime}}
$$

is a cocycle, and the following is immediate:

Proposition 2.22. Let $G=G(r, s, t, f)$ be an abelian metacyclic group, and assume without loss of generality that $t=1$ and $f=(r, s, f)$. Then the integral cohomology algebra $\mathrm{H}^{*}(G, \mathbf{Z})$ of $G$ is as an algebra generated by (the classes of $c_{x^{\prime}}, c_{y^{\prime}}$, and $\xi_{3}$, subject to the relations

$$
s^{\prime} c_{x^{\prime}}=0, \quad f c_{y^{\prime}}=0, \quad f \xi_{3}=0, \quad \xi_{3}^{2}=\frac{s^{\prime}\left(s^{\prime}-1\right)}{2} c_{x^{\prime}} c_{y^{\prime}}^{2}
$$

Notice that $\xi_{3}^{2}$ is nonzero only if $s^{\prime}$ is even; it is of course well known that this must be so for formal reasons.

For our purposes it will be convenient to reinterpret the description of the integral cohomology algebra $\mathrm{H}^{*}(G, \mathbf{Z})$ of an abelian metacyclic group $G$ in terms of the old generators $x$ and $y$ :

A straightforward calculation shows that

$$
\inf _{K \uparrow G}\left(c_{x}\right)=c_{y^{\prime}}+\frac{r}{f} c_{x^{\prime}}
$$

where $c_{x} \in \mathrm{H}^{2}(K, \mathbf{Z})$ denotes the obvious generator. Hence, if we write $c_{y}=$ $c_{x^{\prime}}$ and $c_{x}=c_{y^{\prime}}+\frac{r}{f} c_{x^{\prime}}$, as an algebra over $\mathrm{H}^{*}(K, \mathbf{Z})=\mathrm{P}\left[c_{x}\right] / s c_{x}$, the integral cohomology of $G$ is generated by $c_{y}$ and $\xi_{3}$, subject to the relations

$$
r c_{y}=f c_{x}, \quad f \xi_{3}=0, \quad \xi_{3}^{2}=\frac{\frac{r s}{f}\left(\frac{r s}{f}-1\right)}{2} c_{y}\left(c_{x}^{2}+\left(\frac{r}{f}\right)^{2} c_{y}^{2}\right) .
$$


Furthermore, it is clear that $\operatorname{res}_{G \downarrow N}\left(c_{y}\right)=c_{y} \in \mathrm{H}^{2}(N, \mathbf{Z})$ with an abuse of the notation $c_{y}$.

We now return to an arbitrary metacyclic group $G$, given by a presentation (0.1). Our final aim in this section is to examine the cohomology of $G$ at a prime $p$ so that its $p$-Sylow subgroup is abelian. We proceed as follows:

Recall that $\nu=\frac{h_{1} f}{r}$ modulo $q_{1}$ is the class of $\mathbf{e}$ in $\mathrm{H}^{2}(K, N)$.

Proposition 2.25. If $p$ is a prime so that $\nu \not \equiv 0 \bmod \left(q_{1}\right)_{p}$, then $t \equiv 1 \bmod p$, and $p$ divides $r$ and $s$, too.

Notice that $\nu \not \equiv 0 \bmod \left(q_{1}\right)_{p}$ if and only if $f \not \equiv 0 \bmod \left(k_{1}\right)_{p}=(r, s)_{p}$.

Proof of 2.25. Recall that the number $q_{1}=\frac{h_{1} k_{1}}{r}$ is the order of $\mathrm{H}^{2}(K, N)$. It is clear that the hypothesis implies that $p$ divides $q_{1}$. Now this, in turn, implies that $p$ divides $t-1$ as well; in fact, if $p$ did not divide $t-1$, the $p$-primary part of

$$
k_{1}=\left(1+t+\cdots+t^{s-1}, r\right)=\left(\frac{t^{s}-1}{t-1}, r\right)
$$

would coincide with that of $r$ since by hypothesis $t^{s} \equiv 1 \bmod r$ and $p$ would not divide the number $q_{1}=\frac{(t-1, r) k_{1}}{r}$.

For a prime $p$ we shall denote the $p$-Sylow subgroup of $G$ by $G(p)$ (but note that we write $G_{p}$ for the preimage in $G$ of the $p$-Sylow subgroup $K_{p}$ of $K)$.

Lemma 2.26. For a prime $p$ so that $\nu \not \equiv 0 \bmod \left(q_{1}\right)_{p}$, the restriction map, restricted to the p-primary part $\mathrm{H}^{*}(G, \mathbf{Z})_{p}$ of $\mathrm{H}^{*}(G, \mathbf{Z})$, is an isomorphism

$$
\text { res: } \mathrm{H}^{*}(G, \mathbf{Z})_{p} \longrightarrow \mathrm{H}^{*}(G(p), \mathbf{Z}) \text {. }
$$

Proof. We have already observed (cf. (2.21) above) that the restriction map identifies $\mathrm{H}^{*}(G, \mathbf{Z})_{p}$ with the invariants $\mathrm{H}^{*}\left(G_{p}, \mathbf{Z}\right)^{K / K_{p}}$ with respect to the induced action coming from conjugation in $G$. However, since $t \equiv 1 \bmod p$, conjugation in $G$ boils down to inner automorphisms in $G_{p}$ whence $K / K_{p}$ acts trivially on $\mathrm{H}^{*}(G, \mathbf{Z})_{p}$. Finally, since $N$ may be written as a direct product $N=N(p) \times N^{\prime}$, where the order of $N^{\prime}$ is prime to $p$, the group $G_{p}$ maps onto its $p$-Sylow subgroup in such a way that the restriction to this $p$-Sylow subgroup is the identity map. This implies at once that the indicated restriction map is an isomorphism.

Hence we conclude at once the following.

Theorem 2.27. Let $p$ be a prime $p$ so that $\nu \not \equiv 0 \bmod \left(q_{1}\right)_{p}$ and $t \equiv 1 \bmod r_{p}$. Then the $p$-Sylow subgroup $G(p)$ of $G$ is a nonsplit abelian metacyclic group, and the restriction map res: $\mathrm{H}^{*}\left(G_{p}, \mathbf{Z}\right)_{p} \longrightarrow \mathrm{H}^{*}(G(p), \mathbf{Z})$ is an isomorphism at $p$. In particular, at $p$ the structure of $\mathrm{H}^{*}(G, \mathbf{Z})$ is given by (2.22). 


\section{SOME NUMBER THEORY}

In the present section we give some number theory that will be needed later in the paper. For a prime $p$ and a number $m$ we shall denote the $p$-part of $m$ by $m_{p}$ so that $m=m_{p} m^{\prime}$ with $m^{\prime}$ prime to $p$.

Let $p$ be a prime and let $G$ be a metacyclic $p$-group given by a presentation of the kind (0.1) so that in particular the numbers $r$ and $s$ are $p$ th powers. Since a cyclic group of order $p$ does not have a nontrivial automorphism of order $p$, we must have $t \equiv 1 \bmod p$. We shall denote the order of $t$ modulo $r$ by $d$, and we shall refer to the case where $p$ is odd or $p=2$ and $t \equiv 1$ mod 4 as the typical case and to the case where $p=2$ and $t \equiv-1 \bmod 4$ as the exceptional case. As before, we denote the corresponding group extension by $\mathbf{e}_{\nu}$, where $\nu=\frac{f(t-1, r)}{r}$.

Lemma 3.1. (1) In the typical case, for any $j \geq 1,\left(t^{j}-1\right)_{p}=(j(t-1))_{p}$.

(2) In the exceptional case, for $j$ odd, $\left(t^{j}-1\right)_{2}=2$, while for $j$ even, $\left(t^{j}-1\right)_{2}=(j(t+1))_{2}$.

For a proof see, e.g., Lemma 2.3 in Huebschmann [27]. We mention in passing that the lemma implies that in the typical case $s \geq p^{2}$ unless $G$ is abelian, i.e., unless the order $d$ of $t$ modulo $r$ is 1 . In fact, since $q_{1}$ is divisible by $p$ so is $\frac{t^{s}-1}{r}$. However, since $t \equiv 1 \bmod p$, in view of the lemma the $p$-primary parts of $\frac{t^{s}-1}{r}$ and $\frac{s(t-1)}{r}$ coincide; hence the $p$-divisibility of $\frac{t^{s}-1}{r}$ implies that $s=p$ only if $t-1$ is divisible by $r$, i.e., only if $d=1$. On the other hand, in the exceptional case it may well happen that $s=2$ while the 2-Sylow subgroup of $G$ is nonabelian.

The lemma entails (3.2), (3.3), and (3.4) below:

Corollary 3.2. (1) In the typical case, $d=r / h_{1}$ and, for $j \geq 1$,

$$
\begin{gathered}
h_{j}=h_{1}(j, d), \quad k_{j}=(r, s), \\
q_{j}=\frac{h_{1}(r, s)}{r}(j, d)=\frac{(r, s)}{d}(j, d)=q_{1}(j, d) .
\end{gathered}
$$

(2) In the exceptional case, $d=\frac{r}{(t+1, r / 2)}$, and for $j \geq 1$,

$$
\begin{aligned}
& h_{j}= \begin{cases}(t+1)_{2}(j, d), & \text { if } j \text { is even, } \\
2, & \text { if } j \text { is odd, }\end{cases} \\
& k_{j}= \begin{cases}(r, s), & \text { if } j \text { is even, } \\
\left(\frac{s(t+1)}{r}, 2\right) \frac{r}{2}, & \text { if } j \text { is odd, }\end{cases} \\
& q_{j}= \begin{cases}\left(\frac{s(t+1)}{r}, 2\right), & \text { if } j \text { is odd, } \\
\frac{(t+1)_{2}(r, s)}{r}(j, d), & \text { if } j \text { is even. }\end{cases}
\end{aligned}
$$

Details of the calculation of $d$ are given in $\S 3$ of [27]; the other statements are immediate. 
Addendum. In the exceptional case a nonsplit extension occurs only if the number $\frac{(t+1) s}{r}$ is even. Furthermore, for $j$ odd the number $k_{j}$ then coincides with $r$.

Proof. In fact, the order $q_{1}=\left(\frac{s(t+1)}{r}, 2\right)$ of $\mathrm{H}^{2}(K, N)$ is then greater than 1 if and only if the number $\frac{(t+1) s}{r}$ is even. Furthermore, if this happens to be the case, for $j$ odd, we have

$$
k_{j}=\left(\frac{s(t+1)}{r}, 2\right) \frac{r}{2}=r .
$$

Let $G^{\prime}$ be the subgroup of $G$ generated by $y$ and $x^{\prime}=x^{p}$. It is again metacyclic, the obvious presentation being given by

$$
G^{\prime}=G\left(r, s^{\prime}, t^{\prime}, f\right)=\left\langle\tilde{x}, y ; y^{r}=1, x^{s^{\prime}}=y^{f}, x^{\prime} y x^{\prime^{-1}}=y^{t^{\prime}}\right\rangle,
$$

where $s^{\prime}=s / p$ and $t^{\prime}=t^{p}$.

Corollary 3.3. In the typical case, for $j \geq 1$,

$$
\begin{aligned}
& h_{j}^{\prime}= \begin{cases}p h_{j}, & \text { if } j \text { is not divisible by } d, \\
h_{j}, & \text { if } j \text { is divisible by } d,\end{cases} \\
& k_{j}^{\prime}= \begin{cases}\frac{k_{j}}{p}, & \text { if } r \geq s, \\
k_{j}, & \text { if } r<s,\end{cases} \\
& q_{j}^{\prime}= \begin{cases}q_{j}, & \text { if } r \geq s \text { and } j \neq 0 \text { mod } d \text { or if } r<s \text { and } j \equiv 0 \bmod d, \\
\frac{q_{j}}{p}, & \text { if } r \geq s \text { and } j \text { is divisible by } d, \\
p q_{j}, & \text { if } r<s \text { and } j \text { is not divisible by } d .\end{cases}
\end{aligned}
$$

In particular, if $d>1$, i.e., if $G$ is nonabelian,

$$
q_{1}^{\prime}= \begin{cases}q_{1}, & \text { if } r \geq s, \\ p q_{1}, & \text { if } r<s,\end{cases}
$$

and $\nu^{\prime}=p \nu$.

Proof. As an example, we compute $\nu^{\prime}=\frac{h_{1}^{\prime} f}{r}=\frac{p h_{1} f}{r}=p \frac{h_{1} f}{r}=p \nu$. The proof of the other statements is left to the reader.

Corollary 3.4. In the exceptional case, if $j$ is odd,

$$
\begin{aligned}
& h_{j}^{\prime}=\left(t+1, \frac{r}{2}\right) h_{j}= \begin{cases}(t+1)_{2} h_{j}, & \text { if } t+1 \not \equiv 0 \bmod r, \\
\frac{r}{2} h_{j}, & \text { if } t+1 \equiv 0 \bmod r,\end{cases} \\
& k_{j}^{\prime}=\frac{(s, 2 r)}{(s(t+1), 2 r)} k_{j}= \begin{cases}\frac{s}{\left(\frac{s(t+1)}{r}, 2\right) r} k_{j}, & \text { if } r \geq s, \\
k_{j}, & \text { if } r<s,\end{cases} \\
& q_{j}^{\prime}=\frac{s(t+1)_{2}}{r},
\end{aligned}
$$

while if $j$ is even, 
In particular,

$$
\begin{aligned}
& h_{j}^{\prime}= \begin{cases}2 h_{j}, & \text { if } j(t+1) \text { is not divisible by } r, \\
h_{j}, & \text { if } j(t+1) \text { is divisible by } r,\end{cases} \\
& k_{j}^{\prime}= \begin{cases}\frac{k_{j}}{2}, & \text { if } r \geq s, \\
k_{j}, & \text { if } r<s,\end{cases} \\
& q_{j}^{\prime}= \begin{cases}q_{j}, & \text { if } r \geq s \text { and } j(t+1) \not \equiv 0 \bmod r, \\
\frac{q_{j}}{2}, & \text { if } r \geq s \text { and } j(t+1) \equiv 0 \bmod r, \\
2 q_{j}, & \text { if } r<s \text { and } j(t+1) \not \equiv 0 \bmod r, \\
q_{j}, & \text { if } r<s \text { and } j(t+1) \equiv 0 \bmod r .\end{cases}
\end{aligned}
$$

and

$$
q_{1}^{\prime}= \begin{cases}q_{1}=1, & \text { if } r=(t+1)_{2} s, \\ \frac{s(t+1)_{2}}{r} \geq 2, & \text { if } r<(t+1)_{2} s,\end{cases}
$$

$$
\nu^{\prime}=\left(t+1, \frac{r}{2}\right) \nu=\frac{r}{d} \nu \equiv \begin{cases}0 \bmod q_{1}^{\prime}, & \text { if } r \geq s, \\ \frac{q_{1}^{\prime}}{2} \bmod q_{1}^{\prime} & \text { otherwise. }\end{cases}
$$

Proof. As an example, we compute again

$$
\nu^{\prime}=\frac{h_{1}^{\prime} f}{r}=\frac{\left(t+1, \frac{r}{2}\right) h_{1} f}{r}=\left(t+1, \frac{r}{2}\right) \frac{h_{1} f}{r}=\left(t+1, \frac{r}{2}\right) \nu=\frac{r}{d} \nu .
$$

If $r \geq s$, the number $\left(t+1, \frac{r}{2}\right)$ is divisible by $q_{1}^{\prime}=\left(\frac{(t+1) s}{r}, \frac{s}{2}\right)$, whence $\nu^{\prime} \equiv 0$ $\bmod q_{1}^{\prime}$ as asserted. Likewise, if $r<s, \frac{q_{1}^{\prime}}{2}=\left(t+1, \frac{r}{2}\right)$, whence $\nu^{\prime} \equiv 0 \bmod \frac{q_{1}^{\prime}}{2}$ as asserted. The proof of the other statements is again left to the reader.

\section{THE CONSTRUCTION OF GENERATORS}

We now return to an arbitrary metacyclic group $G$ given by a presentation of the kind (0.1), and as before we write $\mathbf{e}_{\nu}$ for the corresponding group extension. In the present section we construct cohomology classes in $\mathrm{H}^{*}(G, \mathbf{Z})$ at a prime $p$ so that neither the $p$-Sylow subgroup of $G$ is abelian nor the restriction of e to the $p$-Sylow subgroup $K_{p}$ of $K$ splits.

For a prime $p$ and a number $m$, as before we denote by $m_{p}$ the $p$-primary part of $m$. We now define the number $\operatorname{ord}_{p}(m)$ by

$$
m_{p}=p^{\operatorname{ord}_{p}(m)} \text {. }
$$

We also recall that, in the standard way, from the cohomology of the extension e the cohomology ring $\mathrm{H}^{*}(G, Z)$ inherits a filtration which is natural in e and, for $n \geq 1$, may be written,

$$
\mathrm{H}^{n}(G, \mathbf{Z})=\mathrm{H}^{0, n}(\mathbf{e}) \supseteq \mathrm{H}^{1, n-1}(\mathbf{e}) \supseteq \cdots \supseteq \mathrm{H}^{n, 0}(\mathbf{e})=\inf \left(\mathbf{H}^{n}(K, \mathbf{Z})\right) ;
$$

as usual, we shall refer to elements in $\mathrm{H}^{i, n-i}(\mathbf{e})$ as having filtration $i$. 
We now pick a prime $p$ so that $\nu \not \equiv 0 \bmod \left(q_{1}\right)_{p}$ and concentrate henceforth at this single prime. In view of Lemma 2.26 we may assume that $G$ is a metacyclic $p$-group, and we shall do so until the end of this section. The significance of this assumption is that it will simplify the exposition somewhat. As before we shall denote the order of $t$ modulo $r_{p}=r$ by $d$. It is clear that $d>1$ if and only if $t \not \equiv 1 \bmod r_{p}$, i.e., if and only if $G$ is nonabelian. As in the previous section, let $G^{\prime}$ be the subgroup of $G$ generated by $y$ and $x^{\prime}=x^{p}$, and let

$$
\mathbf{e}^{\prime}: 1 \rightarrow N \rightarrow G^{\prime} \stackrel{\mathrm{pr}}{\longrightarrow} K^{\prime} \rightarrow 1
$$

be the corresponding group extension, where $K^{\prime}=\left\langle x^{\prime} ; x^{\prime s^{\prime}}=1\right\rangle$. Then the injection maps $\imath: G^{\prime} \longrightarrow G$ and $\imath: K^{\prime} \longrightarrow K$ yield a morphism

$$
(\mathrm{Id}, l, l): \mathbf{e}^{\prime} \longrightarrow \mathbf{e}
$$

of extensions. It is clear that if $d>1$, the order $d^{\prime}$ of $t^{\prime}$ modulo $r$ is given by $d=p d^{\prime}$, and if we iterate this procedure $\operatorname{ord}_{p}(d)$ times, we arrive at the subgroup $\widetilde{G}$ of $G$ generated by $y$ and $\tilde{x}=x^{d}$. This group is abelian and metacyclic, the obvious presentation being given by

$$
\widetilde{G}=G(r, \tilde{s}, \tilde{t}, f)=\left\langle\tilde{x}, y ; y^{r}=1, \tilde{x}^{\tilde{s}}=y^{f}, \tilde{x} y \tilde{x}^{-1}=y\right\rangle,
$$

where $\tilde{s}=s / d$ and $\tilde{t}=t^{d}$, and it fits into the group extension

$$
\tilde{\mathbf{e}}: 1 \rightarrow N \rightarrow \widetilde{G} \stackrel{\mathrm{pr}}{\longrightarrow} \widetilde{K} \rightarrow 1,
$$

where $\widetilde{K}=\left\langle\tilde{x} ; \tilde{x}^{\tilde{s}}=1\right\rangle$. Further, the injection maps yield a series

$$
\tilde{\mathbf{e}} \stackrel{(\mathrm{Id}, l, l)}{\longrightarrow} \cdots \stackrel{(\mathrm{Id}, l, l)}{\longrightarrow} \mathbf{e}^{\prime \prime} \stackrel{(\mathrm{Id}, l, l)}{\longrightarrow} \mathbf{e}^{\prime} \stackrel{(\mathrm{Id}, l, l)}{\longrightarrow} \mathbf{e}
$$

of morphisms of extensions.

Our next aim is to construct cohomology classes for $G$. To this end we reproduce briefly the construction of the unitary representation $\alpha: G \longrightarrow U(d)$ given in Huebschmann [27]: Pick a primitive complex $r$ th root of unity $z_{y}$ and, furthermore, a primitive complex root of unity $z_{x}$ of the appropriate order so that $z_{x}^{s}=z_{y}^{f}$. Let

$$
\alpha(y)=\left[\begin{array}{ccccc}
z_{y} & 0 & 0 & \cdots & 0 \\
0 & z_{y}^{t} & 0 & \cdots & 0 \\
\cdot & \cdot & \cdot & \cdots & \cdot \\
\cdot & \cdot & \cdot & \cdots & \cdot \\
\cdot & \cdot & \cdot & \cdots & 0 \\
0 & 0 & 0 & \cdots & z_{y}^{t^{d-1}}
\end{array}\right] \in U(d)
$$




$$
\alpha(x)=\left[\begin{array}{ccccc}
0 & z_{x} & 0 & \cdots & 0 \\
0 & 0 & 0 & \cdots & 0 \\
\cdot & \cdot & \cdot & \cdots & \cdot \\
\cdot & \cdot & \cdot & \cdots & \cdot \\
\cdot & \cdot & \cdot & \cdots & z_{x} \\
z_{x} & 0 & 0 & \cdots & 0
\end{array}\right] \in U(d)
$$

It is not hard to see that this yields indeed a unitary representation

$$
\alpha: G \longrightarrow U(d)
$$

details may be found in [27]. For $1 \leq j \leq d$, we write $c_{j}(\alpha) \in \mathrm{H}^{2 j}(G, \mathbf{Z})$ for the $j$ th Chern class of $\alpha$. Accordingly, we write $z_{x^{\prime}}=z_{x}^{p}, z_{\tilde{x}}=z_{x}^{d}$, and we denote the corresponding unitary representations of $G^{\prime}$ and $\widetilde{G}$ by $\alpha^{\prime}: G^{\prime} \longrightarrow U\left(\tilde{d}^{\prime}\right)$ and $\tilde{\alpha}: \widetilde{G} \longrightarrow U(\tilde{d})$, respectively.

We now write $c_{\tilde{x}}, \tilde{\zeta}_{2}=c_{y}$, and $\tilde{\xi}_{3}$ for the corresponding generators given in $\S 2$ and recall that, by virtue of (2.22), as an algebra, the integral cohomology $\mathrm{H}^{*}(\widetilde{G}, \mathbf{Z})$ is generated by these classes subject to the relations

$$
\begin{gathered}
r \tilde{\zeta}_{2}=(r, \tilde{s}, f) c_{\tilde{x}}, \quad \tilde{s} c_{\tilde{x}}=0 \\
(r, \tilde{s}, f) \tilde{\xi}_{3}=0, \quad \tilde{\xi}_{3}^{2}=\frac{s^{b}\left(s^{b}-1\right)}{2} \tilde{\zeta}_{2}\left(c_{\tilde{x}}^{2}+\left(\frac{r}{(r, f, \tilde{s})}\right)^{2} \tilde{\zeta}_{2}^{2}\right),
\end{gathered}
$$

spelled out as $(2.24)$, where $s^{b}=\frac{r \tilde{s}}{(r, \tilde{s}, f)}$. Notice that in this case

$$
\tilde{h}_{j}=r, \quad \tilde{\nu}=f, \quad\left(\tilde{q}_{1}, \tilde{\nu}\right)=(r, \tilde{s}, f) .
$$

For $j>1$, let

$$
\tilde{\xi}_{2 j+1}=\tilde{\zeta}_{2}^{j-1} \tilde{\xi}_{3} \in \mathrm{H}^{2 j+1}(\widetilde{G}, \mathbf{Z}), \quad \tilde{\zeta}_{2 j}=\tilde{\zeta}_{2}^{j} \in \mathrm{H}^{2 j}(\widetilde{G}, \mathbf{Z}) .
$$

Then it is clear that, as a graded module over the cohomology ring $\mathrm{H}^{*}(\widetilde{K}, \mathbf{Z})$ of the quotient group $\widetilde{K}=\mathbf{Z} / \tilde{s}$, the integral cohomology $\mathrm{H}^{*}(\widetilde{G}, \mathbf{Z})$ is generated by the classes $\tilde{\zeta}_{2 j}$ and $\tilde{\xi}_{2 j+1}$.

We now carry out our construction of cohomology classes for $G$. As before we shall denote the transfer in group cohomology from a subgroup $H$ of $G$ by $\tau_{H \uparrow G}$. . We begin with the construction of odd degree generators in the typical case:

If $f_{p} \leq \tilde{s}=\frac{s}{d}$, for $1 \leq j \leq d$, let

$$
\xi_{2 j+1}=\tau_{\widetilde{G} \uparrow G}\left(\tilde{\xi}_{2 j+1}\right) \in \mathrm{H}^{2 j+1}(G, \mathbf{Z}) .
$$

However, if $f_{p}>\tilde{s}=\frac{s}{d}$, tracing (4.6) backwards we will encounter a split extension $\mathbf{e}^{(n)}$ before we reach $\tilde{\mathbf{e}}$, and we proceed otherwise; we shall comment on this in (4.20) below: Let

$$
\sigma=\frac{s}{f_{p}}=\frac{q_{1}}{\left(\nu, q_{1}\right)} \frac{s}{(r, s)},
$$


and let $G_{\sigma}$ be the subgroup of $G$ generated by $y$ and $x_{\sigma}=x^{\sigma}$. It is still metacyclic, the obvious presentation being given by

$$
G_{\sigma}=G\left(r, f_{p}, t^{\sigma}, f\right)=\left\langle x_{\sigma}, y ; y^{r}=1, x_{\sigma}^{f_{p}}=y^{f}, x_{\sigma} y x_{\sigma}^{-1}=y^{t^{\sigma}}\right\rangle,
$$

and it fits into the group extension

$$
\mathbf{e}_{\sigma}: 1 \rightarrow N \rightarrow G_{\sigma} \stackrel{\mathrm{pr}}{\longrightarrow} K_{\sigma} \rightarrow 1,
$$

where $K_{\sigma}$ is the subgroup of $K$ generated by $x_{\sigma}$. Furthermore, it is clear that the order $d_{\sigma}$ of $t^{\sigma}$ modulo $r$ is given by $d=\sigma d_{\sigma}$, and by hypothesis $d_{\sigma}>1$. By construction it is clear that the extension $\mathbf{e}_{\sigma}$ splits. In fact, write

$$
\left(1+\left(t^{\sigma}\right)+\cdots+\left(t^{\sigma}\right)^{f_{p}-1}, r\right)=U\left(1+\left(t^{\sigma}\right)+\cdots+\left(t^{\sigma}\right)^{f_{p}-1}\right)+V r ;
$$

then $x_{\sigma} y^{-U f / f_{p}} \in G_{\sigma}$ has exact order $f_{p}$. For $1 \leq j \leq d$, we now take

$$
\xi_{2 j+1}=\tau_{G_{\sigma} \uparrow G}\left(\left(\xi_{2 j+1}\right)_{\sigma}\right) \in \mathrm{H}^{2 j+1}(G, \mathbf{Z}),
$$

where the classes $\left(\xi_{2 j+1}\right)_{\sigma}$ refer to the corresponding generators given in (2.8).

In the exceptional case, we observe first that, since $t^{\prime}=t^{2} \equiv 1 \bmod 4$, the restricted case $\mathbf{e}^{\prime}$ is typical; hence we may assume that classes

$$
\xi_{2 j+1}^{\prime} \in \mathrm{H}^{2 j+1}\left(G^{\prime}, \mathbf{Z}\right)
$$

have already been constructed. For $1 \leq j \leq d$, we then take

$$
\xi_{2 j+1}=\tau_{G^{\prime} \uparrow G}\left(\xi_{2 j+1}^{\prime}\right) \in \mathrm{H}^{2 j+1}(G, \mathbf{Z}) .
$$

We record that, by construction, for $1 \leq j \leq d$,

$$
\operatorname{res}_{G \downarrow G^{\prime}}\left(\xi_{2 j+1}\right)=\left(1+x+\cdots+x^{p-1}\right) \xi_{2 j+1}^{\prime} .
$$

The construction of the even degree classes is more complicated and proceeds by induction as follows: Consider the extension (4.1) labelled $\mathrm{e}^{\prime}$ above, and suppose by induction that classes $\zeta_{2 j}^{\prime} \in \mathrm{H}^{2 j}\left(G^{\prime}, \mathbf{Z}\right)$ have already been constructed. The induction starts at $\widetilde{G}$. For $1 \leq j<d$, let

$$
\zeta_{2 j}=\tau_{G^{\prime} \uparrow G}\left(\zeta_{2 j}^{\prime}\right) \in \mathrm{H}^{2 j}(G, \mathbf{Z}),
$$

unless we are in the exceptional case with $j$ odd; then it is clear that

$$
\operatorname{res}_{G \downarrow G^{\prime}}\left(\zeta_{2 j}\right)=\left(1+x+\cdots+x^{p-1}\right) \zeta_{2 j}^{\prime} \text {. }
$$

In the exceptional case with $j$ odd we proceed as follows: Recall that the modulo 2 Bockstein exact sequence looks like

$$
\cdots \rightarrow \mathrm{H}^{2 j-1}(G, \mathbf{Z} / 2) \stackrel{\beta}{\longrightarrow} \mathrm{H}^{2 j}(G) \rightarrow \mathrm{H}^{2 j}(G) \rightarrow \mathrm{H}^{2 j}(G, \mathbf{Z} / 2) \rightarrow \cdots .
$$

We recall from Theorem $\mathrm{E}$ in our earlier paper [30] that the modulo 2 cohomology of $G$ is as an algebra generated by classes $\omega_{x}, C_{x}, \omega_{1}, \Xi_{3}, C_{4}$, the 
degrees of which are indicated by their subscripts; we have chosen capital letters to avoid conflict with the notation in the present paper. For $j$ odd and $1 \leq j<d$ we now take

$$
\zeta_{2 j}=\beta\left(\omega_{1} C_{4}^{\frac{j-1}{2}}\right) \in \mathrm{H}^{2 j}(G) ;
$$

by construction it is then clear that $\zeta_{2 j}$ has at most order 2 and inspection of the commutative diagram

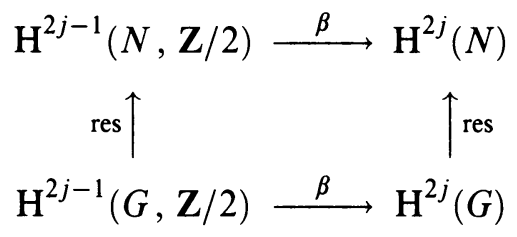

then shows that

$$
\operatorname{res}\left(\zeta_{2 j}\right)=\frac{r}{2} c_{y}^{j}
$$

In particular, $\zeta_{2 j}$ has exact order 2 .

Next, returning to the general case, in dimension $2 d$ we take

$$
\zeta_{2 d}=t^{-b} c_{2 d}(\alpha) \in \mathrm{H}^{2 d}(G, \mathbf{Z}),
$$

where $t^{-b}$ is defined by the requirement that $t^{b}=t^{d^{\prime}} \frac{p(p-1)}{2}$; this makes sense since the latter is a unit modulo $p$. By Corollary 4.5 of [27] we then have res $_{G \downarrow G^{\prime}}\left(\zeta_{2 d}\right)=\zeta_{2 d}^{\prime}$, whence

$$
\tau_{G^{\prime} \uparrow G}\left(\zeta_{2 d}^{\prime}\right)=p \zeta_{2 d}
$$

Finally, if $j>d$, write $j=k d+i, 0 \leq i<d$, and let

$$
\begin{aligned}
\xi_{2 j+1} & = \begin{cases}\tilde{\zeta}_{2 d}^{k-1} \tilde{\xi}_{2 d+1} \in \mathrm{H}^{2 j+1}(G, \mathbf{Z}), & \text { if } i=0, \\
\zeta_{2 d}^{k} \xi_{2 i+1} \in \mathrm{H}^{2 j+1}(G, \mathbf{Z}), & \text { if } i>0,\end{cases} \\
\zeta_{2 j} & =\zeta_{2 d}^{k} \zeta_{2 i} \in \mathrm{H}^{2 j}(G, \mathbf{Z}) .
\end{aligned}
$$

By construction the classes $\zeta_{2 j}$ and $\xi_{2 j+1}$ have filtration 0 and 1 respectively. For later reference we record the following:

Proposition 4.21. (1) For $j \geq 1$, $\operatorname{res}_{G \downarrow G^{\prime}}\left(\xi_{2 j+1}\right)=\left(1+x+\cdots+x^{p-1}\right) \xi_{2 j+1}^{\prime}$.

(2) For $j \geq 1, \tau_{G^{\prime} \uparrow G}\left(\xi_{2 j+1}^{\prime}\right)=\xi_{2 j+1}^{\prime}$.

(3) In the typical case, for $j \geq 1$, $\operatorname{res}_{G \downarrow G^{\prime}}\left(\zeta_{2 j}\right)=\left(1+x+\cdots+x^{p-1}\right) \zeta_{2 j}^{\prime}$, whenever $j$ is not divisible by $d$, while $\operatorname{res}_{G \downarrow G^{\prime}}\left(\zeta_{2 j d}\right)=\zeta_{2 j d}^{\prime}$.

(4) In the exceptional case, for $j$ even, $\operatorname{res}_{G \downarrow G^{\prime}}\left(\zeta_{2 j}\right)=(1+x) \zeta_{2 j}^{\prime}$, whenever $j(t+1)$ is not divisible by $r$, while $\operatorname{res}_{G \downarrow G^{\prime}}\left(\zeta_{2 j}\right)=\zeta_{2 j}^{\prime}$ if $j(t+1)$ is divisible by $r$. 
(5) In the exceptional case, for $j$ odd, the class $\zeta_{2 j}$ has exact order 2.

Finally we spell out some useful information about the integral cohomology spectral sequence $E_{\mu}^{*, *}\left(\mathbf{e}, d_{\mu}\right)$ of the group extension $\mathbf{e}$ which may be deduced from the above construction of generators. The notation will be the same as that in (2.1); we remind the reader that (2.1) gives $E_{2}$ in the general case.

Proposition 4.22. (1) For $j \geq 1$, the class $\operatorname{res}_{G \downarrow N}\left(\zeta_{2 j}\right)$ coincides with $c_{2 j}=\frac{r}{h_{j}} c_{y}^{j}$, perhaps up to a unit at $p$. Consequently in the cohomology spectral sequence $E_{\mu}^{*, *}\left(\mathbf{e}, d_{\mu}\right)$, the graded group $E_{2}^{\text {even even }}$ consists of infinite cycles only.

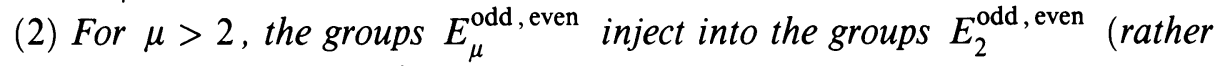
than into a quotient thereof).

(3) Under the combined map

$$
\mathrm{H}^{1,2 j}(G, \mathbf{Z}) \rightarrow E_{\infty}^{1,2 j}(\mathbf{e}, \mathbf{Z}) \rightarrow E_{2}^{1,2 j}
$$

of this injection with the obvious surjection $\mathrm{H}^{1,2 j}(G, \mathbf{Z}) \longrightarrow E_{\infty}^{1,2 j}(\mathbf{e}, \mathbf{Z})$ the classes $\xi_{2 j+1}$ are mapped to

$$
\frac{q_{1}}{\left(\nu, q_{1}\right)} \frac{r}{k_{j}} \omega_{x} c_{y}^{j} \in E_{2}^{1,2 j}=\mathrm{H}^{1}\left(K, \mathrm{H}^{2 j}(N, \mathbf{Z})\right) .
$$

In particular, the latter are infinite cycles.

Notice that in the exceptional case $q_{1}=2,\left(\nu, q_{1}\right)=1$, and that in view of (2.1), for $j$ odd, the above says that the classes $\xi_{2 j+1}$ are mapped to $0 \in$ $E_{2}^{1,2 j}=\mathrm{H}^{1}\left(K, \mathrm{H}^{2 j}(N, \mathbf{Z})\right)$.

Proof. Repeated application of the relevant statements in (4.21) (involving the classes $\left.\zeta_{2 j}\right)$ and (3.1) shows that, for $j \geq 1$, the class $\operatorname{res}_{G \downarrow N}\left(\zeta_{2 j}\right)$ coincides with $c_{2 j}=\frac{r}{h_{j}} c_{y}^{j}$, perhaps up to a unit at $p$. Hence, in view of the multiplicative structure, a formal spectral sequence argument shows at once that $E_{2}^{\text {even, even }}$ consists of infinite cycles only. This establishes (1). Likewise, since (1) has already been verified, a formal argument shows that, for $\mu>2$, the groups $E_{\mu}^{\text {odd, even }}$ inject into the groups $E_{2}^{\text {odd even }}$, whence (2) holds.

We verify statement (3) by induction. We observe first that, by construction, for $j \geq 1$,

$$
\tilde{\xi}_{2 j+1}=\frac{r}{(r, \tilde{s}, f)} \omega_{x} c_{y}^{j}+\text { terms of higher filtration. }
$$

Now $\tilde{q}_{1}=(r, \tilde{s})=\tilde{k}_{j}$ and $\left(\tilde{\nu}, \tilde{q}_{1}\right)=(r, \tilde{s}, f)$, whence indeed

$$
\tilde{\xi}_{2 j+1}=\frac{\tilde{q}_{1}}{\left(\tilde{\nu}, \tilde{q}_{1}\right)} \frac{r}{\tilde{k}_{j}} \omega_{x} c_{y}^{j}+\text { terms of higher filtration. }
$$

Likewise, leaving aside the exceptional case for the moment, assume that $f_{p}>$ $\tilde{s}=\frac{s}{d}$, and consider the above split extension $\mathbf{e}_{\sigma}$, where $\sigma=\frac{s}{f_{p}}$. The corresponding classes $\left(\xi_{2 j+1}\right)_{\sigma}$ have been defined by means of $(2.3)$, from which we deduce the requisite statement right away. Hence the induction starts. 
Still being in the typical case, we now assume that under the corresponding morphism $\left(4.23^{\prime}\right.$ ) (where " ' " refers to the morphism (4.23) for the extension $\left.\mathbf{e}^{\prime}\right)$ the classes $\xi_{2 j+1}^{\prime}$ are mapped to

$$
\frac{q_{1}^{\prime}}{\left(\nu^{\prime}, q_{1}^{\prime}\right)} \frac{r}{k_{j}^{\prime}} \omega_{x^{\prime}} c_{y}^{j} \in E_{2}^{1,2 j}\left(\mathbf{e}^{\prime}, \mathbf{Z}\right)=\mathrm{H}^{1}\left(K^{\prime}, \mathrm{H}^{2 j}(N, \mathbf{Z})\right) .
$$

This implies that, for $1 \leq j \leq d$, under (4.23) the class $\xi_{2 j+1}=\tau_{G^{\prime} \dagger G}\left(\xi_{2 j+1}^{\prime}\right)$ goes to

$$
\frac{q_{1}^{\prime}}{\left(\nu^{\prime}, q_{1}^{\prime}\right)} \tau_{K^{\prime} \uparrow K}\left(\frac{r}{k_{j}^{\prime}} \omega_{x^{\prime}} c_{y}^{j}\right) \in E_{2}^{1,2 j}(\mathbf{e}, \mathbf{Z})=\mathrm{H}^{1}\left(K, \mathrm{H}^{2 j}(N, \mathbf{Z})\right) .
$$

To compute this class in terms of the undashed data, we observe first that, in view of (3.3),

$$
\frac{q_{1}^{\prime}}{\left(\nu^{\prime}, q_{1}^{\prime}\right)} \frac{r}{k_{j}^{\prime}}=\left\{\begin{array}{cl}
\frac{q_{1}}{\left(p \nu, q_{1}\right)} \frac{r p}{k_{j}}, & \text { if } r \geq s \\
\frac{p q_{1}}{\left(p \nu, p q_{1}\right)} \frac{r}{k_{j}}, & \text { if } r<s
\end{array}\right\}=\frac{q_{1}}{\left(\nu, q_{1}\right)} \frac{r}{k_{j}} .
$$

Furthermore, a calculation on the $E_{1}$-level shows that

$$
\tau_{K^{\prime} \uparrow K}\left(\omega_{x^{\prime}} c_{y}^{j}\right)=\omega_{x} c_{y}^{j} \in E_{1}^{1,2 j}(\mathbf{e}, \mathbf{Z}) .
$$

For completeness we reproduce it here: Take

$$
\begin{aligned}
E_{1}^{*, 2 j}\left(\mathbf{e}^{\prime}, \mathbf{Z}\right) & =\operatorname{Hom}_{K^{\prime}}\left(\mathbf{M}(K), \mathrm{H}^{2 j}(N, \mathbf{Z})\right), \\
E_{1}^{*, 2 j}(\mathbf{e}, \mathbf{Z}) & =\operatorname{Hom}_{K}\left(\mathbf{M}(K), \mathbf{H}^{2 j}(N, \mathbf{Z})\right),
\end{aligned}
$$

and let

$$
\Omega: \mathbf{M}(K)_{1}=\mathbf{Z} K \longrightarrow \mathrm{H}^{2 j}(N, \mathbf{Z})
$$

be the cochain which sends 1 to 1 and is zero on the other generators of $\mathbf{Z K}$ as an abelian group. Then $\omega_{x^{\prime}}$ and $\omega_{x}$ may be written

$$
\omega_{x^{\prime}}=\left(1+x^{\prime}+\cdots+x^{\prime s^{\prime}-1}\right) \Omega, \quad \omega_{x}=\left(1+x+\cdots+x^{s-1}\right) \Omega,
$$

whence

Hence $\xi_{2 j+1}$ behaves as asserted.

$$
\omega_{x}=\left(1+x+\cdots+x^{p-1}\right) \omega_{x^{\prime}}=\tau_{K^{\prime} \uparrow K}\left(\omega_{x^{\prime}}\right) .
$$

In the exceptional case, the restricted case $\mathbf{e}^{\prime}$ is typical, as we have already seen. Assume first that $r \geq s$; in view of (3.4) the extension $\mathbf{e}^{\prime}$ then splits, and under $\left(4.23^{\prime}\right)$ the class $\xi_{2 j+1}^{\prime}$ goes to

$$
\frac{r}{k_{j}^{\prime}} \omega_{x^{\prime}} c_{y}^{j} \in E_{2}^{1,2 j}\left(\mathbf{e}^{\prime}, \mathbf{Z}\right)=\mathrm{H}^{1}\left(K^{\prime}, \mathrm{H}^{2 j}(N, \mathbf{Z})\right) .
$$

However, $q_{1}=2$ and, in view of (3.4),

$$
\frac{r}{k_{j}^{\prime}}= \begin{cases}q_{1} \frac{r}{k_{j}}, & \text { if } j \text { is even, } \\ q_{1} \frac{r}{s}, & \text { if } j \text { is odd }\end{cases}
$$


whence, for $1 \leq j \leq d$, under (4.23) the class $\xi_{2 j+1}=\tau_{G^{\prime} \uparrow G}\left(\xi_{2 j+1}^{\prime}\right)$ goes to

$$
\tau_{K^{\prime} \uparrow K}\left(q_{1} \frac{r}{k_{j}} \omega_{x^{\prime}} c_{y}^{j}\right)=q_{1} \frac{r}{k_{j}} \omega_{x} c_{y}^{j} \in E_{2}^{1,2 j}(\mathbf{e}, \mathbf{Z})=\mathbf{H}^{1}\left(K, \mathrm{H}^{2 j}(N, \mathbf{Z})\right),
$$

if $j$ is even, and to

$$
\tau_{K^{\prime} \uparrow K}\left(q_{1} \frac{r}{s} \omega_{x^{\prime}} c_{y}^{j}\right)=q_{1} \frac{r}{s} \omega_{x} c_{y}^{j} \in E_{2}^{1,2 j}(\mathbf{e}, \mathbf{Z})=\mathrm{H}^{1}\left(K, \mathrm{H}^{2 j}(N, \mathbf{Z})\right)
$$

otherwise; however, the latter is zero in view of the description of $E_{2}$ given in (2.1). Finally, we assume that $r<s$. Since the restricted case $\mathrm{e}^{\prime}$ is typical, by virtue of what has already been proved, under $\left(4.23^{\prime}\right)$ the classes $\xi_{2 j+1}^{\prime}$ are mapped to

$$
\frac{q_{1}^{\prime}}{\left(\nu^{\prime}, q_{1}^{\prime}\right)} \frac{r}{k_{j}^{\prime}} \omega_{x^{\prime}} c_{y}^{j} \in E_{2}^{1,2 j}\left(\mathbf{e}^{\prime}, \mathbf{Z}\right)=\mathrm{H}^{1}\left(K^{\prime}, \mathrm{H}^{2 j}(N, \mathbf{Z})\right) .
$$

However, $q_{1}=2$ and, in view of (3.4),

$$
\frac{q_{1}^{\prime}}{\left(\nu^{\prime}, q_{1}^{\prime}\right)} \frac{r}{k_{j}^{\prime}}=\frac{\left(r, s^{\prime}\right)}{d} \frac{q_{1}}{\left(t+1, \frac{r}{2}\right)} \frac{r}{k_{j}^{\prime}}=2,
$$

whence, for $1 \leq j \leq d$, under (4.23) the class $\xi_{2 j+1}=\tau_{G^{\prime} \uparrow G}\left(\xi_{2 j+1}^{\prime}\right)$ goes to

$$
\tau_{K^{\prime} \uparrow K}\left(q_{1} \omega_{x^{\prime}} c_{y}^{j}\right)=q_{1} \omega_{x} c_{y}^{j} \in E_{2}^{1,2 j}(\mathbf{e}, \mathbf{Z})=\mathrm{H}^{1}\left(K, \mathrm{H}^{2 j}(N, \mathbf{Z})\right) .
$$

For $j$ odd, in view of the description of $E_{2}$ given in (2.1) the latter is zero, while for $j$ even, in view of (3.2), $k_{j}=(r, s)=r$, whence $q_{1}=q_{1} \frac{r}{k_{j}}$.

Finally, an argument involving the multiplicative structure completes the proof. We leave the details to the reader.

Remark 4.24. If $f_{p}>\tilde{s}=\frac{s}{d}$, we cannot recover the infinite cycles

$$
\frac{q_{1}}{\left(\nu, q_{1}\right)} \frac{r}{k_{j}} \omega_{x} c_{y}^{j} \in E_{2}^{1,2 j}=\mathrm{H}^{1}\left(K, \mathrm{H}^{2 j}(N, \mathbf{Z})\right)
$$

by means of the transfer from the cohomology of the subgroup $\widetilde{G}$ (given by (4.4) above). This explains why under these circumstances we constructed the classes $\xi_{2 j+1}$ by means of the transfer from the cohomology of the subgroup $G_{\sigma}$ (given by (4.11) above). On the other hand, still in the situation where $f_{p}>\tilde{s}$, we have, up to a unit,

$$
\tau_{\widetilde{G} \uparrow G_{\sigma}}\left(\tilde{\zeta}_{2 j}\right)=\cdot\left(\zeta_{2 j}\right)_{\sigma}, \text { if } j \not \equiv 0 \bmod p
$$

unless $p=2, r>2, t \equiv-1 \bmod r$, and $j$ is odd, $\tau_{\widetilde{G} \uparrow G_{\sigma}}\left(\tilde{\zeta}_{2 p}\right)=p\left(\zeta_{2 p}\right)_{\sigma}$, etc.

Remark 4.25. In Theorem B of [27] we proved that $E_{2}^{\text {even, even }}$ consists of infinite cycles only by an argument different from the one given above.

\section{Characteristic Classes}

In this section we compute certain characteristic classes introduced in [26]. Under the present circumstances these classes govern the first nonzero differentials in the integral homology and cohomology spectral sequences of the group 
extension (0.2). In (5.1) some preliminaries will be given. In (5.2) we review briefly the characteristic classes. In (5.3) we compute the Ext-groups in which the characteristic classes assume their values. In (5.4) we compute the homology characteristic classes and in (5.5) the cohomology characteristic classes.

5.1. Preliminaries. As already indicated, we now take the ground ring $R$ to be the integers $Z$. To have available an appropriate language, we need some preparations:

With the notation introduced in $\S 1$ above, the standard small free resolution for a cyclic group $Q=\left\langle z ; z^{a}\right\rangle$ may be described as the chain complex that underlies the differential graded algebra

$$
(\mathbf{M}(Q), d)=\left(\Gamma\left[u_{z}\right] \otimes \Lambda\left[v_{z}\right]\right) \otimes_{d} \mathbf{Z} Q
$$

whose differential $d$ is given by

$$
\begin{aligned}
& d\left(\gamma_{i+1}\left(u_{z}\right)\right)=\left(\gamma_{i}\left(u_{z}\right)\right) v_{z}\left(1+z+\cdots+z^{a-1}\right), \\
& d\left(\gamma_{i}\left(u_{z}\right)\right) v_{z}=\left(\gamma_{i}\left(u_{z}\right)\right)(z-1), \quad i \geq 0
\end{aligned}
$$

here and henceforth we discard the tensor product symbol and write $u_{z} v_{z}=$ $u_{z} \otimes v_{z}$, etc. It will be convenient to write $\mathbf{M}^{\sharp}(Q)=\Gamma\left[u_{z}\right] \otimes \Lambda\left[v_{z}\right]$ and $\mathbf{M}(Q)=$ $\mathbf{M}^{\sharp}(Q) \otimes_{d} R Q$.

The dual $\mathrm{M}(Q)^{*}$ of $\mathrm{M}(Q)$ may be written $\mathrm{M}(Q)^{*}=\mathrm{P}\left[c_{z}\right] \otimes \Lambda\left[\omega_{z}\right] \otimes(\mathbf{Z} Q)^{*}$, where $c_{z}$ and $\omega_{z}$ denote the duals of $u_{z}$ and $v_{z}$ in the basis of monomials, and where $\mathrm{P}\left[c_{z}\right]$ is the polynomial algebra on $c_{z}$. It is well known that, with an abuse of notation, as a ring, $\mathrm{H}^{*} N=\mathrm{P}\left[c_{y}\right]$, while as a graded module, for $i \geq 1, \mathrm{H}_{2 i-1} N=(\mathrm{Z} / r)\left\langle\left(\gamma_{i-1}\left(u_{y}\right)\right) v_{y}\right\rangle$ and $\mathrm{H}_{2 i} N=0$.

At the risk of making a mountain of a molehill we recall that the conjugation action of $G$ on $N$ given by $w \mapsto x w x^{-1}, w \in N$, induces an action of $K=\left\langle x ; x^{s}\right\rangle$ on the left of $N$ and hence on the left of $\mathrm{H}_{*} N$ and the right of $\mathrm{H}^{*} N$. We note that, $K$ being abelian, the distinction between "left" and "right" amounts to a consistent choice of signs. These actions of $K$ on $\mathrm{H}^{*} N$ and $\mathrm{H}_{*} N$ are given by the rules

$$
c_{y} \cdot x=t c_{y}, \quad x \cdot\left(\left(\gamma_{i-1} u_{y}\right) v_{y}\right)=t^{i}\left(\gamma_{i-1} u_{y}\right) v_{y}, \quad i>0 .
$$

It will be convenient to treat $\mathrm{H}_{*} N$ as a right $K$-module as well, with respect to

$$
\left(\left(\gamma_{i-1} u_{y}\right) v_{y}\right) \cdot x=t^{-i}\left(\gamma_{i-1} u_{y}\right) v_{y}, \quad i>0
$$

we note that $t^{-i}$ makes sense modulo $r$.

Let $A$ be an abelian group whose exponent divides the number $t^{s}-1$, e.g., $A=\mathbf{Z} / r$. For $j \in \mathbf{Z}$, we shall write $A(j)$ for the $\mathbf{Z} K$-module which as an abelian group is just $A$ and whose $K$-structure is given by letting the generator $x$ of $K=\mathbf{Z} / s$ act by multiplication with $t^{j}$. Notice this makes sense for negative $j$ also if we use the convention $A(-1)=A(s-1)$, etc. With this 
notation it is clear that, for $j \geq 1$, as right $K$-modules,

$$
\begin{aligned}
& \mathrm{H}^{2 j-1} N \cong(\mathbf{Z} / r)(j), \\
& \mathrm{H}^{2 j} N \cong(\mathbf{Z} / r)(j), \\
& \mathrm{H}_{2 j-1} N \cong(\mathbf{Z} / r)(-j), \\
& \mathrm{H}_{2 j} N \cong(\mathbf{Z} / r)(-j) .
\end{aligned}
$$

For later reference we record the following:

(5.1.6) When $(A(j))^{\otimes i}$ is equipped with the diagonal action then

$$
(A(j))^{\otimes i}=\left(A^{\otimes i}\right)(i j) .
$$

(5.1.7) When $\operatorname{Hom}(A(i), A(j))$ is equipped with the diagonal action, that is, for $y \in A(i)$ and $\phi \in \operatorname{Hom}(A(i), A(j))$, the result $(x \phi) y$ is given by

$$
(x \phi) y=x \phi\left(x^{-1} y\right) \text { or }(\phi x) y=\left(\phi\left(y x^{-1}\right)\right) \text {, }
$$

according as $A(i)$ and $A(j)$ are viewed as left or right $(\mathbf{Z K})$-modules, then

$$
\operatorname{Hom}(A(i), A(j))=(\operatorname{Hom}(A, A))(j-i) .
$$

5.2. Characteristic classes. We denote the integral homology and cohomology spectral sequences of the group extension $(0.2)$ by $\left(E_{p q}^{r}, d^{r}\right)$ and $\left(E_{r}^{p q}, d_{r}\right)$, respectively. In [26] we introduced suitable characteristic classes. Since the integral homology of a finite cyclic group vanishes in even positive degrees and since its integral cohomology vanishes in odd degrees, under the present circumstances these classes read

$$
v_{3}^{q} \in \operatorname{Ext}_{K}^{3}\left(\mathrm{H}^{q}(N), \mathrm{H}^{q-2}(N)\right), \quad q \geq 2,
$$

and

$$
w_{2}^{0} \in \operatorname{Ext}_{K}^{2}\left(\mathrm{H}_{0}(N), \mathrm{H}_{1}(N)\right), \quad w_{3}^{q} \in \operatorname{Ext}_{K}^{3}\left(\mathrm{H}_{q}(N), \mathrm{H}_{q+2}(N)\right), \quad q \geq 1 .
$$

Moreover, there are pairings

$$
\begin{aligned}
& \mu: E_{3}^{p, q} \otimes \operatorname{Ext}_{K}^{3}\left(\mathrm{H}^{q}(N), \mathrm{H}^{q-2}(N)\right) \longrightarrow E_{3}^{p+3, q-2}, \quad q \geq 1, \\
& \mu: \operatorname{Ext}_{K}^{2}\left(\mathrm{H}_{0}(N), \mathrm{H}_{1}(N)\right) \otimes E_{p, 0}^{2} \longrightarrow E_{p-2,1}^{2}, \\
& \mu: \operatorname{Ext}_{K}^{3}\left(\mathrm{H}_{q}(N), \mathrm{H}_{q+2}(N)\right) \otimes E_{p, q}^{3} \longrightarrow E_{p-3, q+2}^{3}, \quad q \geq 1,
\end{aligned}
$$

so that, up to sign, for $p \geq 2$, the differential $d^{2}: E_{p, 0}^{2} \rightarrow E_{p-2,1}^{2}$ is given by

$$
d^{2}=\mu\left(w_{2}^{0} \otimes-\right): E_{p, 0}^{2} \longrightarrow E_{p-2,1}^{2},
$$

and so that, for $q \geq 1$ and $p$ as appropriate, up to sign, the differentials $d_{3}$ and $d^{3}$ are given by

$$
d_{3}=\mu\left(-\otimes v_{3}^{q}\right): E_{3}^{p, q} \longrightarrow E_{3}^{p+3, q-2}, \quad d^{3}=\mu\left(w_{3}^{q} \otimes-\right): E_{p, q}^{3} \longrightarrow E_{p-3, q+2}^{3}
$$

More details may be found in [26]. 
5.3. Free resolutions for the (co)homology of $N$. In the present subsection we compute the Ext groups in which our characteristic classes attain their values. We do this by means of appropriate free resolutions. For brevity, we restrict attention to those Ext groups needed later in the paper. However, it would be easy to push further and give a complete computation of, e.g., $\operatorname{Ext}_{\mathbf{Z} K}^{k}((\mathbf{Z} / r)(j),(\mathbf{Z} / r)(j+l))$ for any $j, k, l$. For convenience, we shall write $\mathrm{H}_{*}(N)=\mathrm{H}_{*}(N, \mathbf{Z})$ and $\mathrm{H}^{*}(N)=\mathrm{H}^{*}(N, \mathbf{Z})$, and we shall treat them both as right $(\mathbf{Z} K)$-modules, as indicated in (5.1) above.

For $j \geq 0$, let

$$
\begin{array}{ll}
a_{j}^{+}=\frac{\left(t^{j}\right)^{s}-1}{r}, & b_{j}^{+}=\left(t^{j}\right)^{s-1}+\left(t^{j}\right)^{s-2} x+\cdots+x^{s-1}, \\
a_{j}^{-}=-a^{+}, & b_{j}^{-}=1+\left(t^{j} x\right)+\cdots+\left(t^{j} x\right)^{s-1} .
\end{array}
$$

Then

$$
\begin{aligned}
b_{j}^{+}\left(x-t^{j}\right) & =1-\left(t^{j}\right)^{s}=-r a_{j}^{+}, \\
b_{j}^{-}\left(t^{j} x-1\right) & =\left(t^{j}\right)^{s}-1=-r a_{j}^{-} .
\end{aligned}
$$

We remind the reader that the numbers $h_{j}, k_{j}, q_{j}$ have been defined in the Introduction.

Let $j \geq 0$. Using, e.g., a filtration argument, it is not hard to see that the chain complex

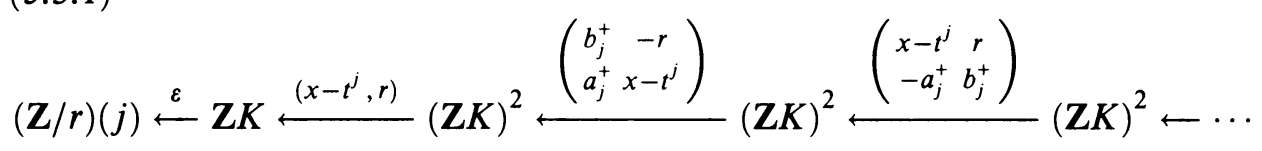

is a free resolution of $(\mathbf{Z} / r)(j)$ in the category of right $(\mathbf{Z} K)$-modules. Likewise, the chain complex

$$
\begin{aligned}
(\mathbf{Z} / r)(-j)) & \stackrel{\varepsilon}{\leftarrow} \mathbf{Z} K \stackrel{\left(t^{j} x-1, r\right)}{\stackrel{(}{(} K)^{2}} \\
& \stackrel{\left(\begin{array}{cc}
b_{j}^{-} & -r \\
a_{j}^{-} & t^{j} x-1
\end{array}\right)}{\longleftarrow}(\mathbf{Z} K)^{2} \stackrel{\left(\begin{array}{cc}
t^{j} x-1 & r \\
-a_{j}^{-} & b_{j}^{-}
\end{array}\right)}{\longleftarrow}(\mathbf{Z} K)^{2} \leftarrow \cdots
\end{aligned}
$$

is a free resolution of $(\mathbf{Z} / r)(-j)$ in the category of right $(\mathbf{Z} K)$-modules. Notice that for $j=0$ the two resolutions coincide. We mention that here the elements of $(\mathbf{Z K})^{2}$ are identified in the usual way with column vectors with two entries from $\mathbf{Z} K$, and the differential in the resolutions is given by the usual matrix multiplication $\left(\begin{array}{c}* * \\ * *\end{array}\right)\left(\begin{array}{l}* \\ *\end{array}\right)=\left(\begin{array}{l}* \\ *\end{array}\right)$.

Proposition 5.3.3. (1) With reference to the trivial $K$-action on $\mathbf{Z}$, the group $\operatorname{Ext}_{\mathbf{Z K}}^{3}((\mathbf{Z} / r)(1), \mathbf{Z})$ is cyclic of order $q_{1}$, generated by the class $\tau_{3}$ of the cocycle

$$
\left(\frac{1-t}{h_{1}}, \frac{r}{h_{1}}\right):(\mathbf{Z} K)^{2} \longrightarrow \mathbf{Z} .
$$


(2) For $j \geq 1$, the group $\operatorname{Ext}_{\mathbf{Z} K}^{3}((\mathbf{Z} / r)(j),(\mathbf{Z} / r)(j-1))$ is isomorphic to $\left(\mathbf{Z} / q_{1}\right) \times\left(\mathbf{Z} / q_{1}\right)$, generated by the classes $\tilde{\tau}_{3}^{j}$ and $\tau_{3}^{j}$ of the cocycles

$$
\left(\frac{r}{k_{1}}, 0\right):(\mathbf{Z} K)^{2} \longrightarrow(\mathbf{Z} / r)(j-1)
$$

and

$$
\left(j \frac{1-t}{h_{1}}, \frac{r}{h_{1}}\right):(\mathbf{Z} K)^{2} \longrightarrow(\mathbf{Z} / r)(j-1),
$$

respectively.

(3) For $j \geq 0$, the group $\operatorname{Ext}_{\mathbf{Z} K}^{3}((\mathbf{Z} / r)(-j),(\mathbf{Z} / r)(-j-1))$ is isomorphic to $\left(\mathbf{Z} / q_{1}\right) \times\left(\mathbf{Z} / q_{1}\right)$, generated by the classes $\tilde{\vartheta}_{3}^{j}$ and $\vartheta_{3}^{j}$ of the cocycles

$$
\left(\frac{r}{k_{1}}, 0\right):(\mathbf{Z} K)^{2} \longrightarrow(\mathbf{Z} / r)(-j-1)
$$

and

$$
\left(j \frac{t-1}{h_{1}}, \frac{r}{h_{1}}\right):(\mathbf{Z} K)^{2} \longrightarrow(\mathbf{Z} / r)(-j-1)
$$

respectively.

Remark 5.3.4. Since $q_{1} r / k_{1}=h_{1}=(t-1, r)$, for any $l$ and $j$, the class $\tau_{3}^{j}$ is as well represented by the cocycles

$$
\left(t^{l} j \frac{1-t}{h_{1}}, \frac{r}{h_{1}}\right):(\mathbf{Z} K)^{2} \longrightarrow(\mathbf{Z} / r)(j-1)
$$

and

$$
\left(t^{l} \frac{1-t^{j}}{h_{1}}, \frac{r}{h_{1}}\right):(\mathbf{Z} K)^{2} \longrightarrow(\mathbf{Z} / r)(j-1) .
$$

Likewise, for any $l$ and $j$, the class $\vartheta_{3}^{j}$ is also represented by the cocycles

$$
\left(t^{l} j \frac{t-1}{h_{1}}, \frac{r}{h_{1}}\right):(\mathbf{Z} K)^{2} \longrightarrow(\mathbf{Z} / r)(-j-1)
$$

and

$$
\left(t \frac{t^{j}-1}{h_{1}}, \frac{r}{h_{1}}\right):(\mathbf{Z} K)^{2} \longrightarrow(\mathbf{Z} / r)(-j-1) .
$$

Similarly, for any $l$, the class $\tau_{3}$ is represented by the cocycle

$$
\left(t^{l} \frac{1-t}{h_{1}}, \frac{r}{h_{1}}\right):(\mathbf{Z} K)^{2} \longrightarrow \mathbf{Z} .
$$

Proof of 5.3.3. The group $\operatorname{Ext}_{\mathbf{Z} K}^{3}((\mathbf{Z} / r)(1), \mathbf{Z})$ is the homology of

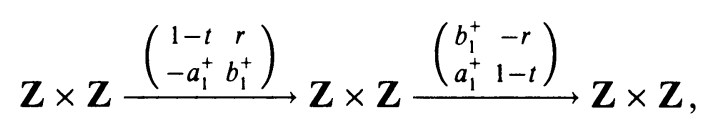


where $a_{1}^{+}=\frac{t^{s}-1}{r}$ and, with a slight abuse of notation, $b_{1}^{+}=1+t+\cdots+t^{s-1}$. Now the kernel of $\left(\begin{array}{cc}b_{1}^{+} & -r \\ a_{1}^{+} & 1-t\end{array}\right)$ is generated by $\left(\frac{1-t}{h_{1}}, \frac{r}{h_{1}}\right)$, and the image of $\left(\begin{array}{cc}1-t & r \\ -a_{1}^{+} & b_{1}^{+}\end{array}\right)$ is generated by

$$
\left(\frac{k_{1}(1-t)}{r}, k_{1}\right)=q_{1}\left(\frac{1-t}{h_{1}}, \frac{r}{h_{1}}\right) \text {. }
$$

This proves (1).

Likewise, for $j \geq 2$, the group $\operatorname{Ext}_{\mathbf{Z} K}^{3}((\mathbf{Z} / r)(j),(\mathbf{Z} / r)(j-1))$ is the homology of

$$
(\mathbf{Z} / r) \times(\mathbf{Z} / r) \stackrel{\left(\begin{array}{cc}
t^{j-1}-t^{j} & 0 \\
-a_{j}^{+} & b_{j}^{+}
\end{array}\right)}{\longrightarrow}(\mathbf{Z} / r) \times(\mathbf{Z} / r) \stackrel{\left(\begin{array}{ll}
b_{j}^{+} & 0 \\
a_{j}^{+} t^{j-1}-t^{j}
\end{array}\right)}{\longrightarrow}(\mathbf{Z} / r) \times(\mathbf{Z} / r),
$$

where, with a slight abuse of notation,

$$
\begin{aligned}
a_{j}^{+} & =\frac{t^{j s}-1}{r}=j \frac{t^{s}-1}{r} \in \mathbf{Z} / r \\
b_{j}^{+} & =t^{j(s-1)}+t^{j(s-2)+(j-1)}+\cdots+t^{(j-1)(s-1)} \\
& =t^{1-j}\left(1+t+\cdots+t^{s-1}\right) \in \mathbf{Z} / r .
\end{aligned}
$$

Now the kernel of $\left(\begin{array}{cc}b_{j}^{+} & 0 \\ a_{j}^{+} t^{j-1}-t^{j}\end{array}\right)$ is generated by $\left(\frac{r}{k_{1}}, 0\right)$ and $\left(t^{j-1} j \frac{1-t}{h_{1}}, \frac{r}{h_{1}}\right)$, the image of $\left(\begin{array}{cc}t^{j-1}-t^{j} & 0 \\ -a_{j}^{+} & b_{j}^{+}\end{array}\right)$is generated by $\left(t^{j-1}-t^{j}, 0\right)$, and

$$
\begin{aligned}
\left(-a_{j}^{+}, b_{j}^{+}\right) & =\left(-j \frac{t^{s}-1}{r}, t^{1-j}\left(1+t+\cdots+t^{s-1}\right)\right) \\
& =t^{1-j}\left(-j t^{j-1}\left(\frac{t^{s}-1}{r}\right),\left(1+t+\cdots+t^{s-1}\right)\right) .
\end{aligned}
$$

Since $h_{1}=(t-1, r), k_{1}=\left(1+t+\cdots+t^{s-1}, r\right)$, and $q_{1}=\frac{h_{1} k_{1}}{r}$, the image of

$$
\left(\begin{array}{cc}
t^{j-1}-t^{j} & 0 \\
-a_{j}^{+} & b_{j}^{+}
\end{array}\right)
$$

is also generated by $\left(h_{1}, 0\right)=q_{1}\left(\frac{r}{k_{1}}, 0\right)$ and

$$
\left(t^{j-1} j \frac{1-t}{k_{1}}, k_{1}\right)=q_{1}\left(t^{j-1} j \frac{1-t}{h_{1}}, \frac{r}{h_{1}}\right) \text {. }
$$

In view of (5.3.4) this proves (2).

The proof of (3) is virtually identical to that of (2). Details are left to the reader.

It seems worthwhile relating the groups $\operatorname{Ext}_{\mathrm{ZK}}^{3}\left(\mathrm{H}^{2 j} N, \mathrm{H}^{2 j-2} N\right), j \geq 1$, with appropriate Ext groups defined over $\mathbf{Z} / r$; this will be relevant for the calculation 
of the class $v_{3}^{2}(\mathbf{e}, \mathbf{Z})$ : It is readily seen that, for $j \in \mathbf{Z}$, (5.3.5)

$$
0 \longleftarrow(\mathbf{Z} / r)(j) \stackrel{\varepsilon}{\longleftarrow}(\mathbf{Z} / r) K \stackrel{x-t^{j}}{\longleftarrow}(\mathbf{Z} / r) K \stackrel{b_{j}^{+}}{\longleftarrow}(\mathbf{Z} / r) K \stackrel{x-t^{j}}{\longleftarrow}(\mathbf{Z} / r) K \stackrel{b_{j}^{+}}{\longleftarrow} \cdots
$$

is a free resolution of $(\mathbf{Z} / r)(j)$ in the category of right $(\mathbf{Z} / r) K$-modules. Hence Proposition 5.3.6. For $j \in \mathbf{Z}$ and $i \geq 1$, the groups

$$
\operatorname{Ext}_{(\mathbf{Z} / r) K}^{i}((\mathbf{Z} / r)(j),(\mathbf{Z} / r)(j-1))
$$

are cyclic of order $q_{1}$, generated by the classes $\left[\frac{r}{h_{1}}\right]$ for $i$ even and $\left[\frac{r}{k_{1}}\right]$ for $i$ odd of the cocycles of (5.3.5) which send $1 \in(\mathbf{Z} / r) K$ to $\frac{r}{h_{1}}$ and $\frac{r}{k_{1}}$ respectively, as indicated.

The suspension $\mathrm{s}(5.3 .5)$ of the free resolution (5.3.5) of

$$
\mathrm{H}^{2 j-1}(N, \mathbf{Z} / r)=(\mathbf{Z} / r)(j)
$$

may be embedded into $(\mathbf{Z} / r) \otimes$ (5.3.1) by means of the map which in dimensions $\geq 1$ sends the generator 1 of $(\mathbf{Z} / r) K$ to $\left(\begin{array}{l}0 \\ 1\end{array}\right) \in((\mathbf{Z} / r) K)^{2}$, and there results the short exact sequence

$$
0 \longrightarrow s(5.3 .5) \longrightarrow(\mathbf{Z} / r) \otimes(5.3 .1) \longrightarrow(5.3 .5) \longrightarrow 0
$$

of chain complexes in the category of $(\mathrm{Z} / r) K$-modules. Here the quotient (5.3.5) is to be viewed as a free resolution of $\mathrm{H}^{2 j}(N, \mathbf{Z} / r)=(\mathbf{Z} / r)(j)$ in the category of right $(\mathrm{Z} / r) K$-modules. Notice that the long exact homology sequence of (5.3.7) yields

$$
\begin{aligned}
\mathrm{H}_{0}((\mathbf{Z} / r) \otimes(5.3 .1)) & \cong \mathrm{H}_{0}((5.3 .5))=\mathrm{H}^{2 j}(N, \mathbf{Z} / r), \\
\mathrm{H}^{2 j-1}(N, \mathbf{Z} / r) & =\mathrm{H}_{1}(\mathrm{~s}(5.3 .5)) \cong \mathrm{H}_{1}((\mathbf{Z} / r) \otimes(5.3 .1)) ;
\end{aligned}
$$

now by standard homological algebra

$$
\mathbf{H}_{1}((\mathbf{Z} / r) \otimes(5.3 .1))=\operatorname{Tor}_{\mathbf{Z}}^{1}\left(\mathbf{Z} / r, \mathrm{H}^{2 j} N\right),
$$

and (5.3.7) identifies in particular the two groups $\mathrm{H}^{2 j-1}(N, \mathrm{Z} / r)$ and $\operatorname{Tor}_{\mathbf{Z}}^{1}\left(\mathbf{Z} / r, \mathrm{H}^{2 j} N\right)$.

In dimensions $>0$, part of the long exact homology sequence of (5.3.7) with coefficients in any $(\mathbf{Z} / r) K$-module $U$ looks like

$$
\begin{aligned}
& \operatorname{Ext}_{(\mathbf{Z} / r) K}^{i}\left(\mathrm{H}^{2 j} N, U\right) \stackrel{l}{\rightarrow} \operatorname{Ext}_{\mathbf{z} K}^{i}\left(\mathrm{H}^{2 j} N, U\right) \\
& \stackrel{\kappa}{\rightarrow} \operatorname{Ext}_{(\mathbf{Z} / r) K}^{i-1}\left(\mathrm{H}^{2 j-1}(N, \mathbf{Z} / r), U\right) ;
\end{aligned}
$$

notice that since $U$ is supposed to be an $(\mathrm{Z} / r) K$-module, the obvious maps $\mathrm{H}^{i}\left(K, \operatorname{Hom}\left(\mathrm{H}^{2 j} N, U\right)\right)=\operatorname{Ext}_{\mathbf{Z} K}^{i}\left(\mathbf{Z} / r, \operatorname{Hom}\left(\mathrm{H}^{2 j} N, U\right)\right) \longrightarrow \operatorname{Ext}_{\mathbf{Z} K}^{i}\left(\mathbf{H}^{2 j} N, U\right)$ and

$$
\begin{aligned}
& \mathrm{H}^{i-1}\left(K, \operatorname{Hom}\left(\mathrm{H}^{2 j-1}, U\right)\right) \\
& \quad=\operatorname{Ext}_{\mathbf{Z K}}^{i-1}\left(\mathbf{Z} / r, \operatorname{Hom}\left(\mathrm{H}^{2 j-1}, U\right)\right) \longrightarrow \operatorname{Ext}_{(\mathbf{Z} / r) K}^{i-1}\left(\mathrm{H}^{2 j-1}, U\right)
\end{aligned}
$$


are isomorphisms of $(\mathbf{Z} / r)$-modules; here, for short, we have written $\mathrm{H}^{2 j-1}=\mathrm{H}^{2 j-1}(N, \mathrm{Z} / r)$.

Proposition 5.3.9. (1) The composite

$$
\begin{aligned}
\operatorname{Ext}_{\mathbf{Z} K}^{3}\left(\mathrm{H}^{2} N, \mathrm{H}^{0} N\right) & \rightarrow \operatorname{Ext}_{\mathbf{Z} K}^{3}\left(\mathrm{H}^{2} N, \mathrm{H}^{0}(N, \mathbf{Z} / r)\right) \\
& \stackrel{\kappa}{\rightarrow} \operatorname{Ext}_{(\mathbf{Z} / r) K}^{2}\left(\mathrm{H}^{1}(N, \mathbf{Z} / r), \mathrm{H}^{0}(N, \mathbf{Z} / r)\right)
\end{aligned}
$$

sends $\tau_{3}^{1}$ to $\left[\frac{r}{h_{1}}\right]$.

(2) For $j \geq 2$ and

$$
U=\mathrm{H}^{2 j-2} N=\mathrm{H}^{2 j-2}(N, \mathbf{Z} / r)=(\mathbf{Z} / r)(j-1),
$$

we have $\kappa\left(\tau_{3}^{j}\right)=\left[\frac{r}{h_{1}}\right]$ and $l\left(\left[\frac{r}{k_{1}}\right]\right)=\tilde{\tau}_{3}^{j}$, where the tacit identifications $\mathrm{H}^{2 j} N=\mathrm{H}^{2 j}(N, \mathbf{Z} / r)=(\mathbf{Z} / r)(j)$ are made.

Notice that, in particular, for $j \geq 2$, there results a split short exact sequence

$$
\begin{aligned}
0 & \rightarrow \operatorname{Ext}_{R K}^{3}\left(\mathrm{H}^{2 j} N, \mathrm{H}^{2 j-2} N\right) \rightarrow \operatorname{Ext}_{\mathrm{ZK}}^{3}\left(\mathrm{H}^{2 j} N, \mathrm{H}^{2 j-2} N\right) \\
& \rightarrow \operatorname{Ext}_{R K}^{2}\left(\mathrm{H}^{2 j-1}(N, R), \mathrm{H}^{2 j-2} N\right) \rightarrow 0,
\end{aligned}
$$

where we have written $R=\mathbf{Z} / r$ for short.

Proof. The statements as to $\tau_{3}^{j}$ follow at once from (i) the commutativity of the diagram

$$
\begin{aligned}
& (\mathbf{Z} / r) K \stackrel{x-t^{j}}{\longleftarrow}(\mathbf{Z} / r) K \stackrel{b_{j}}{\longleftarrow} \quad(\mathbf{Z} / r) K \\
& \left(\begin{array}{l}
0 \\
1
\end{array}\right) \downarrow \quad\left(\begin{array}{l}
0 \\
1
\end{array}\right) \downarrow \quad\left(\begin{array}{l}
0 \\
1
\end{array}\right) \downarrow
\end{aligned}
$$

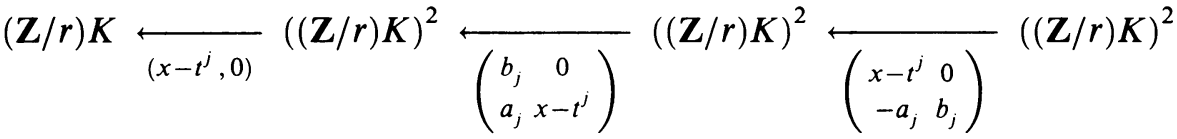

which displays the embedding of $\mathrm{s}(5.3 .5)$ into $(\mathrm{Z} / r) \otimes(5.3 .1)$, and (ii) the definition of the cocycle representing $\tau_{3}^{j}$ : In fact, $\left(t^{j-1} j \frac{1-t}{h_{1}}, \frac{r}{h_{1}}\right)$ represents $\tau_{3}^{j}$, and

$$
\left(t^{j-1} j \frac{1-t}{h_{1}}, \frac{r}{h_{1}}\right)\left(\begin{array}{l}
0 \\
1
\end{array}\right)=\frac{r}{h_{1}} \text {. }
$$

Likewise in each dimension the projection $(\mathbf{Z} / r) \otimes(5.3 .1) \longrightarrow(5.3 .5)$ is given by the matrix $\left(\begin{array}{ll}1 & 0\end{array}\right)$, and the cocycle $\left(\begin{array}{lll}1 & 0\end{array}\right) \frac{r}{k_{1}}=\left(\frac{r}{k_{1}} 0\right)$ represents the class $\tilde{\tau}_{3}^{j}$.

5.4. The characteristic classes for homology. In this subsection we exploit the recipe (3.5) in [26] to compute the homology characteristic classes. As in $\S 2$ (cf. what is said after (2.14)), we shall write $\nu=\frac{f(t-1, r)}{r}$, where $f$ is the number occurring in the presentation (0.1). For convenience, we proceed with right modules rather than left ones, and we depict diagrams with right arrows rather than with left arrows as we did in $\S 3$ of [26]. 
We shall write $\mathrm{H}_{*}(N)=\mathrm{H}_{*}(N, Z)$. Since the integral homology of $N$ is trivial in even positive degrees it is clear that, for $j>0$, the classes

$$
w_{2}^{j}\left(\mathbf{e}_{\nu}, \mathbf{Z}\right) \in \operatorname{Ext}_{\mathbf{Z} K}^{2}\left(\mathrm{H}_{j} N, \mathrm{H}_{j+1} N\right)
$$

are zero, and hence the classes

$$
w_{3}^{j}\left(\mathbf{e}_{\nu}, \mathbf{Z}\right) \in \operatorname{Ext}_{\mathbf{z K}}^{3}\left(\mathrm{H}_{j} N, \mathrm{H}_{j+2} N\right)
$$

are defined for $j>0$ and nonzero at most for $j$ odd. Before we spell out the main result of the present subsection we recall that the standard small free resolution of the integers $\mathbf{Z}$ in the category of right $(\mathbf{Z K})$-modules looks like

$$
0 \stackrel{\varepsilon}{\longleftarrow} \mathbf{Z} \stackrel{x-1}{\longleftarrow} \mathbf{Z} K \stackrel{1+x+\cdots+x^{s-1}}{\longleftarrow} \mathbf{Z} K \stackrel{x-1}{\longleftarrow} \mathbf{Z} K \longleftarrow \cdots .
$$

In particular, with the obvious identifications

$$
\mathrm{H}_{0}(N)=\mathbf{Z} \text { and } \mathrm{H}_{1}(N)=(\mathbf{Z} / r)(-1),
$$

the group $\operatorname{Ext}_{\mathrm{ZK}}^{2}\left(\mathrm{H}_{0} N, \mathrm{H}_{1} N\right)$ is cyclic of order $q_{1}$, generated by the class $\left[\frac{r}{h_{1}}\right]$ of the cocycle of (5.4.1) which sends $1 \in \mathbf{Z} K$ to $\frac{r}{h_{1}} \in(\mathbf{Z} / r)(-1)$.

Theorem 5.4.2. The possibly nonzero characteristic classes in the homology of the group extension $\mathbf{e}_{\nu}$ with integer coefficients are given by

(1) $w_{2}^{0}\left(\mathbf{e}_{\nu}, \mathbf{Z}\right)=\nu\left[\frac{r}{h_{1}}\right] \in \operatorname{Ext}_{\mathbf{Z} K}^{2}\left(\mathrm{H}_{0} N, \mathrm{H}_{1} N\right)=\left(\mathbf{Z} / q_{1}\right)\left\langle\left[\frac{r}{h_{1}}\right]\right\rangle$, and

(2) $w_{3}^{2 j-1}\left(\mathbf{e}_{\nu}, \mathbf{Z}\right)=\nu \vartheta_{3}^{j} \in \operatorname{Ext}_{\mathbf{Z K}}^{3}\left(\mathrm{H}_{2 j-1} N, \mathrm{H}_{2 j+1} N\right)$

$$
=\left(\mathbf{Z} / q_{1}\right)\left\langle\tilde{\vartheta}_{3}^{j}\right\rangle \times\left(\mathbf{Z} / q_{1}\right)\left\langle\vartheta_{3}^{j}\right\rangle \text {, where } j \geq 1 \text {. }
$$

Proof. To prove (1) we denote as usual the augmentation ideal, i.e., the kernel of $\varepsilon: \mathbf{Z} G \rightarrow \mathbf{Z}$, by $I G$ and observe that in $I G \otimes_{\mathbf{Z} N} \mathbf{Z}$ the relation

$$
\left(1+x+\cdots+x^{s-1}\right)((x-1) \otimes 1)=f((y-1) \otimes 1)=\nu \frac{r}{h_{1}(r)}((y-1) \otimes 1)
$$

holds, whence a corresponding diagram $[26,(3.7)]$ looks like

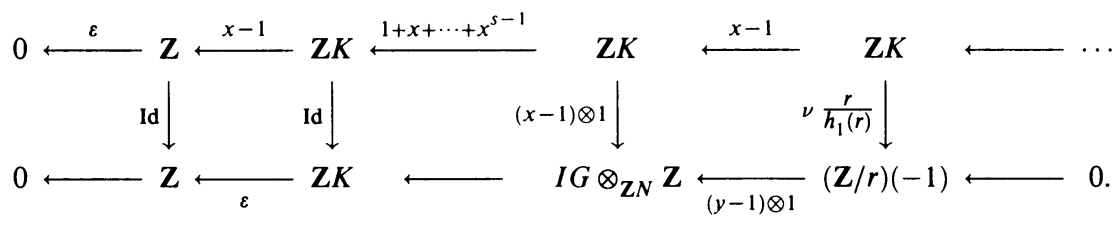

In view of recipe (3.5) in [26], this proves at once statement (1).

To prove (2) we construct a corresponding diagram [26, (3.8)] for $s=3$ as follows: Consider the chain complex $\mathbf{M}(G) \otimes_{\mathbf{Z N}} \mathbf{Z}$ arising as indicated from the Wall resolution $\mathbf{M}(G)$ for $G$ given in $\S 1$. It is clear that, for $j \geq 1$, the chain $\left(\gamma_{j-1}\left(u_{y}\right)\right) v_{y}$ is a cycle, and that

$$
\mathrm{H}_{2 j-1}\left(\mathbf{M}(G) \otimes_{\mathbf{Z N}} \mathbf{Z}\right)=\mathrm{H}_{2 j-1} N=(\mathbf{Z} / r)(-j),
$$


generated by the class of $\left(\gamma_{j-1}\left(u_{y}\right)\right) v_{y}$. We now denote the chains and cycles of $\mathbf{M}(G) \otimes_{\mathbf{Z N}} \mathbf{Z}$ by $C_{*}$ and $Z_{*}$ respectively, and we observe that, for $j \geq 1$, $\mathbf{M}(G) \otimes_{\mathbf{Z N}} \mathbf{Z}$ gives rise to an exact sequence

$$
0 \leftarrow \mathrm{H}_{2 j-1} N \leftarrow Z_{2 j-1} \leftarrow C_{2 j} \stackrel{d}{\longleftarrow} C_{2 j+1} \leftarrow Z_{2 j+1} .
$$

For ease of exposition we spell out the crucial step of the proof as the following.

Lemma 5.4.4. Let $j \geq 1$, and define morphisms $\phi_{0}, \phi_{1}, \phi_{2}$, and $\phi_{3}$ by the formulas

$$
\begin{aligned}
\phi_{0} & =\left(\gamma_{j-1}\left(u_{y}\right) v_{y}\right)(\text { matrix with a single entry }), \\
\phi_{1} & =\left(v_{x}\left(\gamma_{j-1}\left(u_{y}\right)\right) v_{y}, \gamma_{j}\left(u_{y}\right)\right), \\
\phi_{2} & =\left(u_{x}\left(\gamma_{j-1}\left(u_{y}\right)\right) v_{y}, v_{x}\left(\gamma_{j}\left(u_{y}\right)\right)\right), \\
\phi_{3}\left(\begin{array}{l}
1 \\
0
\end{array}\right) & =u_{x}\left(\gamma_{j-1}\left(u_{y}\right) v_{y}\right)\left(t^{j} x-1\right)+\frac{t^{j s}-1}{r} v_{x}\left(\gamma_{j}\left(u_{y}\right)\right), \\
\phi_{3}\left(\begin{array}{l}
0 \\
1
\end{array}\right) & =r u_{x}\left(\gamma_{j-1}\left(u_{y}\right) v_{y}\right)+v_{x}\left(\gamma_{j}\left(u_{y}\right)\right)\left(1+\left(t^{j} x\right)+\cdots+\left(t^{j} x\right)^{s-1}\right) .
\end{aligned}
$$

Then $\left(\phi_{0}, \phi_{1}, \phi_{2}, \phi_{3}\right)$ is a lift (5.3.2) $\longrightarrow(5.4 .3)$ of the identity map, i.e., a commutative diagram

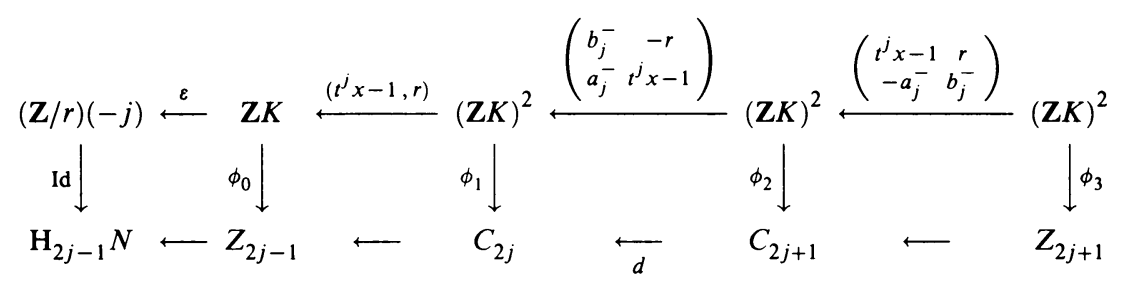

in the category of right $(\mathbf{Z K})$-modules.

Here the free modules $\mathbf{Z} K,(\mathbf{Z} K)^{2}$, etc. are identified with column vectors as usual, and, whenever appropriate, morphisms are given by matrix multiplication $\left(\begin{array}{l}* * \\ * *\end{array}\right)\left(\begin{array}{l}* \\ *\end{array}\right)=\left(\begin{array}{c}* \\ *\end{array}\right)$ as usual.

The lemma is proved by direct inspection; we leave the details to the reader. Notice that the bottom row of (5.4.5) is exact because $N$ has no homology in even degrees. Notice further that the lemma says that our free resolution (5.3.3) of $\mathrm{H}_{2 j-1} N=(\mathrm{Z} / r)(-j)$ recovers a certain piece of the chain complex $\mathbf{M}(G) \otimes_{\mathbf{Z N}} \mathbf{Z}$.

Proof of (5.4.2). By virtue of the formulas for the differential in the Wall reso- 
lution given in (1.1) and (1.2), in $\mathbf{M}(G) \otimes_{\mathbf{Z N}} \mathbf{Z}$ we have

$$
\begin{aligned}
d\left(u_{x} v_{x}\left(\gamma_{j-1}\left(u_{y}\right) v_{y}\right)\right)=\left\{\begin{array}{c}
u_{x}\left(\gamma_{j-1}\left(u_{y}\right) v_{y}\right)\left(t^{j} x-1\right) \\
+\frac{t^{j s}-1}{r} v_{x}\left(\gamma_{j}\left(u_{y}\right)\right)-j t^{j} \frac{(t-1) f}{r}\left(\gamma_{j}\left(u_{y}\right) v_{y}\right),
\end{array}\right. \\
\text { 4.7) } d\left(u_{x}\left(\gamma_{j}\left(u_{y}\right)\right)\right)=\left\{\begin{array}{c}
v_{x}\left(\gamma_{j}\left(u_{y}\right)\right)\left(1+\left(t^{j} x\right)+\cdots+\left(t^{j} x\right)^{s-1}\right) \\
+r u_{x}\left(\gamma_{j-1}\left(u_{y}\right) v_{y}\right)-f\left(\gamma_{j}\left(u_{y}\right)\right) v_{y} .
\end{array}\right.
\end{aligned}
$$

Hence

$$
\phi_{3}\left(\begin{array}{l}
1 \\
0
\end{array}\right)=u_{x}\left(\gamma_{j-1}\left(u_{y}\right) v_{y}\right)\left(t^{j} x-1\right)+\frac{t^{j s}-1}{r} v_{x}\left(\gamma_{j}\left(u_{y}\right)\right)
$$

represents the same homology class in $\mathbf{M}(G) \otimes_{\mathbf{Z N}} \mathbf{Z}$ as

$$
j t^{j} \frac{(t-1) f}{r}\left(\gamma_{j}\left(u_{y}\right) v_{y}\right)=\nu j t^{j} \frac{t-1}{(t-1, r)}\left(\gamma_{j}\left(u_{y}\right)\right) v_{y}
$$

and

$$
\phi_{3}\left(\begin{array}{l}
0 \\
1
\end{array}\right)=r u_{x}\left(\gamma_{j-1}\left(u_{y}\right) v_{y}\right)+v_{x}\left(\gamma_{j}\left(u_{y}\right)\right)\left(1+\left(t^{j} x\right)+\cdots+\left(t^{j} x\right)^{s-1}\right)
$$

represents the same homology class in $\mathbf{M}(G) \otimes_{\mathbf{Z N}} \mathbf{Z}$ as

$$
f\left(\gamma_{j}\left(u_{y}\right)\right) v_{y}=\nu \frac{r}{(t-1, r)}\left(\gamma_{j}\left(u_{y}\right)\right) v_{y} .
$$

In view of Remark 5.3.4, the definition of the classes $\vartheta_{3}^{j}$ and $\tilde{\vartheta}_{3}^{j}$ in (5.3), and recipe (3.5) in [26], we conclude that $w_{3}^{2 j-1}=\nu \vartheta_{3}^{j}$ as asserted.

Remark 5.4.8. Since $N$ is abelian, the class $w_{2}^{0}\left(\mathbf{e}_{\nu}, \mathbf{Z}\right)$ is the usual class of the group extension. In fact, the 2 -fold extension

$$
0 \longleftarrow \mathbf{Z} \stackrel{\varepsilon}{\longleftarrow} \mathbf{Z} K \longleftarrow I G \otimes_{\mathbf{Z} N} \mathbf{Z} \underset{(y-1) \otimes 1}{\longleftarrow}(\mathbf{Z} / r)(-1) \longleftarrow 0
$$

occurring in the proof of (5.4.2(1)) above is just the corresponding object constructed in IV.6 of Mac Lane [39]. Such a remark applies to any group extension e with abelian kernel. This shows how our classes generalize the concept of the class of a group extension with abelian kernel.

To express the requisite computations for cohomology concisely, we now compute our homology characteristic classes with coefficients in $\mathbf{Q} / \mathbf{Z}$, equipped with trivial $G$-action: It is clear that with these coefficients, for $j \geq 1$, the chain $\frac{1}{r} \gamma_{j}\left(u_{y}\right)$ is a cycle, and that

$$
\mathbf{H}_{2 j}\left(\mathbf{M}(G) \otimes_{\mathbf{Z} N}(\mathbf{Q} / \mathbf{Z})\right)=\mathrm{H}_{2 j}(N, \mathbf{Q} / \mathbf{Z})=(\mathbf{Z} / r)(-j)
$$

generated by the class of $\frac{1}{r} \gamma_{j}\left(u_{y}\right)$. Moreover, it is also clear that, for $j \geq 0$,

$$
\mathbf{H}_{2 j+1}\left(\mathbf{M}(G) \otimes_{\mathbf{Z N}}(\mathbf{Q} / \mathbf{Z})\right)=0 \text {. }
$$


Theorem 5.4.9. The characteristic classes $w_{3}^{j}\left(\mathbf{e}_{\nu}, \mathbf{Q} / \mathbf{Z}\right)$ in the homology of the group extension $\mathbf{e}_{\nu}$ with coefficients in $\mathbf{Q} / \mathbf{Z}$ are zero for $j$ odd, while for $j \geq 1$,

$$
\begin{aligned}
w_{3}^{2 j}\left(\mathbf{e}_{\nu}, \mathbf{Q} / \mathbf{Z}\right) & =\nu \vartheta_{3}^{j} \in \operatorname{Ext}_{\mathbf{Z} K}^{3}\left(\mathbf{H}_{2 j}(N, \mathbf{Q} / \mathbf{Z}), \mathrm{H}_{2 j+2}(N, \mathbf{Q} / \mathbf{Z})\right) \\
& =\left(\mathbf{Z} / q_{1}\right)\left\langle\tilde{\vartheta}_{3}^{j}\right\rangle \times\left(\mathbf{Z} / q_{1}\right)\left\langle\vartheta_{3}^{j}\right\rangle .
\end{aligned}
$$

Proof. It is clear that the classes $w_{3}^{j}\left(\mathbf{e}_{\nu}, \mathbf{Q} / \mathbf{Z}\right)$ are zero for $j$ odd. To see that the classes $w_{3}^{2 j}\left(\mathbf{e}_{\nu}, \mathbf{Q} / \mathbf{Z}\right)$ assume the asserted values we proceed as follows: Define morphisms $\phi_{0}^{\prime}, \phi_{1}^{\prime}, \phi_{2}^{\prime}$, and $\phi_{3}$ by the formulas

$$
\begin{aligned}
& \phi_{0}^{\prime}=\left(\frac{1}{r} \gamma_{j}\left(u_{y}\right)\right), \\
& \phi_{1}^{\prime}=\left(\frac{1}{r} v_{x}\left(\gamma_{j}\left(u_{y}\right)\right)\right), \\
& \phi_{2}^{\prime}=\left(\frac{1}{r} u_{x}\left(\gamma_{j}\left(u_{y}\right)\right)+\frac{f}{r^{2}} \gamma_{j+1}\left(u_{y}\right)\right), \\
& \phi_{3}=\left(\frac{1}{r} u_{x}\left(\gamma_{j}\left(u_{y}\right)\right)\left(t^{j} x-1\right)+\frac{f}{r^{2}}\left(\gamma_{j+1}\left(u_{y}\right)\right)\left(t^{j} x-1\right), \frac{f}{r} \gamma_{j+1}\left(u_{y}\right)\right) .
\end{aligned}
$$

Then

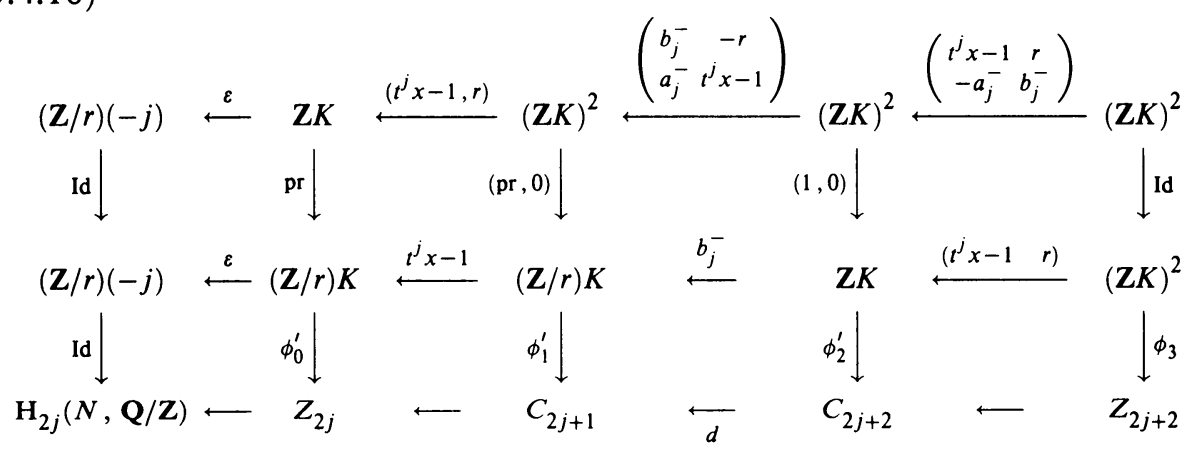

is a commutative diagram in the category of right $(\mathbf{Z K})$-modules. By virtue of the formulas for the differential in the Wall resolution given in (1.1) and (1.2), in $\mathbf{M}(G) \otimes_{\mathbf{Z N}}(\mathbf{Q} / \mathbf{Z})$ we have

$$
\begin{aligned}
& \text { (5.4.11) } d\left(\frac{1}{r} u_{x} v_{x} \gamma_{j}\left(u_{y}\right)\right)=\left\{\begin{array}{c}
\frac{1}{r} u_{x}\left(\gamma_{j}\left(u_{y}\right)\right)\left(t^{j} x-1\right)+\frac{f}{r} v_{x}\left(\gamma_{j}\left(u_{y}\right)\right) v_{y} \\
-(j+1) t^{j} \frac{(t-1) f}{r^{2}}\left(\gamma_{j+1}\left(u_{y}\right)\right) x,
\end{array}\right. \\
& \text { (5.4.12) } d\left(\frac{f}{r^{2}} v_{x} \gamma_{j+1}\left(u_{y}\right)\right)=-\frac{f}{r} v_{x}\left(\gamma_{j}\left(u_{y}\right)\right) v_{y}+\frac{f}{r^{2}}\left(\gamma_{j+1}\left(u_{y}\right)\right)\left(t^{j+1} x-1\right) .
\end{aligned}
$$

Hence the two chains

$$
j t^{j} \frac{(t-1) f}{r} \frac{1}{r} \gamma_{j+1}\left(u_{y}\right) x+\left(t^{j} x-t^{s-1}\right) \frac{(t-1) f}{r} \frac{1}{r} \gamma_{j+1}\left(u_{y}\right)
$$

and

$$
\frac{1}{r} u_{x}\left(\gamma_{j}\left(u_{y}\right)\right)\left(t^{j} x-1\right)+\frac{f}{r^{2}}\left(\gamma_{j+1}\left(u_{y}\right)\right)\left(t^{j} x-1\right)
$$

are equivalent modulo boundaries. Moreover, in view of the right $\mathbf{Z} K$-module structure on

$$
\mathrm{H}_{2 j+2}\left(\mathbf{M}(G) \otimes_{\mathbf{Z N}}(\mathbf{Q} / \mathbf{Z})\right)=\mathrm{H}_{2 j+2}(N, \mathbf{Q} / \mathbf{Z})=(\mathbf{Z} / r)(-j-1)
$$


and, moreover, of Remark 5.3.4,

$$
\begin{gathered}
j t^{j} \frac{(t-1) f}{r} \frac{1}{r} \gamma_{j+1}\left(u_{y}\right) x+\left(t^{j} x-t^{s-1}\right) \frac{(t-1) f}{r} \frac{1}{r} \gamma_{j+1}\left(u_{y}\right) \\
\equiv j t^{s-1} \frac{(t-1) f}{r} \frac{1}{r} \gamma_{j+1}\left(u_{y}\right) \equiv j \frac{(t-1) f}{r} \frac{1}{r} \gamma_{j+1}\left(u_{y}\right),
\end{gathered}
$$

whence

$$
\phi_{3}\left(\begin{array}{l}
1 \\
0
\end{array}\right)=\frac{1}{r} u_{x}\left(\gamma_{j}\left(u_{y}\right)\right)\left(t^{j} x-1\right)+\frac{f}{r^{2}}\left(\gamma_{j+1}\left(u_{y}\right)\right)\left(t^{j} x-1\right)
$$

represents the same homology class of

$$
\mathrm{H}_{2 j+2}\left(\mathbf{M}(G) \otimes_{\mathbf{Z} N}(\mathbf{Q} / \mathbf{Z})\right)=\mathrm{H}_{2 j+2}(N, \mathbf{Q} / \mathbf{Z})
$$

as $j \frac{(t-1) f}{r} \frac{1}{r} \gamma_{j+1}\left(u_{y}\right)$. In view of the definition of the classes $\vartheta_{3}^{j}$ and $\tilde{\vartheta}_{3}^{j}$ in (5.3) and recipe (3.5) in [26], we conclude that

$$
\begin{aligned}
w_{3}^{2 j} & =w_{3}^{2 j}\left(\mathbf{e}_{\nu}, \mathbf{Q} / \mathbf{Z}\right)=\nu \vartheta_{3}^{j} \in \operatorname{Ext}_{\mathbf{Z K}}^{3}\left(\mathbf{H}_{2 j}(N, \mathbf{Q} / \mathbf{Z}), \mathbf{H}_{2 j+2}(N, \mathbf{Q} / \mathbf{Z})\right) \\
& =\left(\mathbf{Z} / q_{1}\right)\left\langle\tilde{\vartheta}_{3}^{j}\right\rangle \times\left(\mathbf{Z} / q_{1}\right)\left\langle\vartheta_{3}^{j}\right\rangle
\end{aligned}
$$

as asserted.

For later reference we record that in terms of the Yoneda theory the class $w_{3}^{2 j}\left(\mathbf{e}_{\nu}, \mathbf{Q} / \mathbf{Z}\right)$ is represented by the 3 -fold extension

$$
0 \leftarrow \mathrm{H}_{2 j} \leftarrow Z_{2 j} \leftarrow C_{2 j+1} \stackrel{d}{\longleftarrow} D_{2 j+3} \leftarrow \mathrm{H}_{2 j+2} \leftarrow 0
$$

where we have written

$$
\begin{aligned}
\mathrm{H}_{2 j} & =\mathrm{H}_{2 j}(N, \mathbf{Q} / \mathbf{Z}), \\
D_{2 j+3} & =\operatorname{coker}\left(d_{2 j+3}\right), \\
\mathrm{H}_{2 j+2} & =\mathrm{H}_{2 j+2}(N, \mathbf{Q} / \mathbf{Z}) .
\end{aligned}
$$

Since the diagram (5.4.9) is commutative, the class $w_{3}^{2 j}\left(\mathbf{e}_{\nu}, \mathbf{Q} / \mathbf{Z}\right)$ is as well represented by the 3 -fold extension

$$
0 \longleftarrow(\mathbf{Z} / r)(-j) \stackrel{\varepsilon}{\longleftarrow}(\mathbf{Z} / r) K \stackrel{t^{j} x-1}{\longleftarrow}(\mathbf{Z} / r) K \stackrel{b_{j}^{b}}{\longleftarrow} D_{2 j+3} \longleftarrow \mathrm{H}_{2 j+2} \longleftarrow 0,
$$

where $b_{j}^{b}$ is induced by $b_{j}^{-}$.

5.5. The characteristic classes for cohomology. In this subsection we exploit Theorem 5.4.9 in order to compute our cohomology characteristic classes. As in the previous subsection, let $\nu=\frac{f(t-1, r)}{r}$, where $f$ is the number occurring in the presentation ( 0.1$)$, and let $\mathbf{e}_{\nu}$ denote the corresponding group extension. We remind the reader that, for degree reasons, the characteristic classes $v_{2}^{k}\left(\mathbf{e}_{\nu}, \mathbf{Z}\right)$ in the cohomology of the group extension $\mathbf{e}_{\nu}$ with integer coefficients are zero, and so are the classes $v_{3}^{2 j+1}\left(\mathbf{e}_{\nu}, \mathbf{Z}\right)$ for $j \geq 0$. 
Theorem 5.5.1. The characteristic classes $v_{3}^{2 j}=v_{3}^{2 j}\left(\mathbf{e}_{\nu}, \mathbf{Z}\right)$ in the cohomology of the group extension $\mathbf{e}_{\nu}$ with integer coefficients are given by

(1) $v_{3}^{2}=-\nu \tau_{3}^{1} \in \operatorname{Ext}_{\mathbf{Z} K}^{3}\left(\mathrm{H}^{2} N, \mathrm{H}^{0} N\right)=\left(\mathbf{Z} / q_{1}\right)\left\langle\tau_{3}^{1}\right\rangle$, and

(2) $v_{3}^{2 j}=-\nu \tau_{3}^{j} \in \mathrm{Ext}_{\mathbf{Z} K}^{3}\left(\mathrm{H}^{2 j} N, \mathrm{H}^{2 j-2} N\right)=\left(\mathbf{Z} / q_{1}\right)\left\langle\tilde{\tau}_{3}^{j}\right\rangle \times\left(\mathbf{Z} / q_{1}\right)\left\langle\tau_{3}^{j}\right\rangle$, where $j \geq 2$.

Proof. Recall the description [26, (2.5)] of the class $v_{3}^{2 j}, j \geq 1$, as the class of a certain 3-fold extension: Let $\varepsilon: \mathbf{P}=\mathbf{P}_{G}(\mathbf{Z}) \rightarrow \mathbf{Z}$ be a free resolution of $\mathbf{Z}$ in the category of $(\mathbf{Z} G)$-modules. Then the chain complex

$\cdots \leftarrow \operatorname{Hom}_{\mathbf{Z} N}\left(P_{2 j+1}, \mathbf{Z}\right) \stackrel{d^{2 j}}{\longleftarrow} \operatorname{Hom}_{\mathbf{Z} N}\left(P_{2 j}, \mathbf{Z}\right) \stackrel{d^{2 j-1}}{\longleftarrow} \operatorname{Hom}_{\mathbf{Z N}}\left(P_{2 j-1}, \mathbf{Z}\right) \leftarrow \cdots$

computes $\mathrm{H}^{*} N$ and inherits a $K$-action. Moreover, since $\mathrm{H}^{*} N$ is zero in odd dimensions, for $j \geq 1$, there results the 3-fold extension

$$
\begin{aligned}
0 & \leftarrow \mathrm{H}^{2 j} N \leftarrow \operatorname{ker}\left(d^{2 j}\right) \leftarrow \operatorname{Hom}_{\mathbf{Z} N}\left(P_{2 j-1}, \mathbf{Z}\right) \\
& \leftarrow \operatorname{coker}\left(d^{2 j-2}\right) \leftarrow \mathrm{H}^{2 j-2} N \leftarrow 0
\end{aligned}
$$

which by means of the Yoneda theory represents the class $v_{3}^{2 j}$.

Let $\left(\phi_{0}, \phi_{1}, \phi_{2}, \phi_{3}\right)$ be a solution of the extension problem

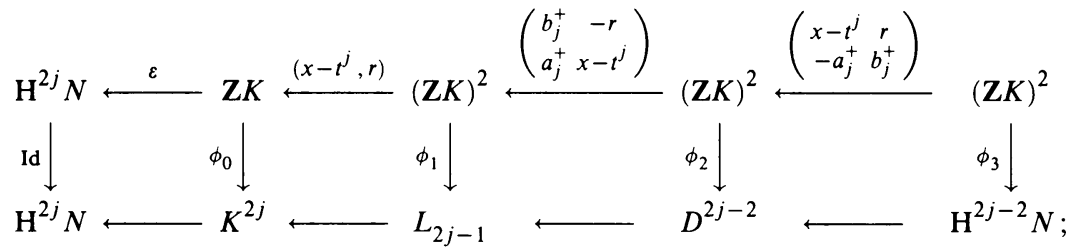

here we have written

$$
K^{2 j}=\operatorname{ker}\left(d^{2 j}\right), \quad L_{2 j-1}=\operatorname{Hom}_{\mathbf{Z N}}\left(P_{2 j-1}, \mathbf{Z}\right), \quad D^{2 j-2}=\operatorname{coker}\left(d^{2 j-2}\right) .
$$

Such a solution exists because the bottom row is acyclic. For convenience, we shall henceforth write $M_{l}=d_{l}\left(P_{l}\right) \subseteq P_{l-1}$; then $\operatorname{ker}\left(d^{2 j}\right)=\operatorname{Hom}_{\mathbf{Z N}}\left(M_{2 j}, \mathbf{Z}\right)$. When we take (5.5.4) modulo $r$ we obtain, in particular, the commutative diagram

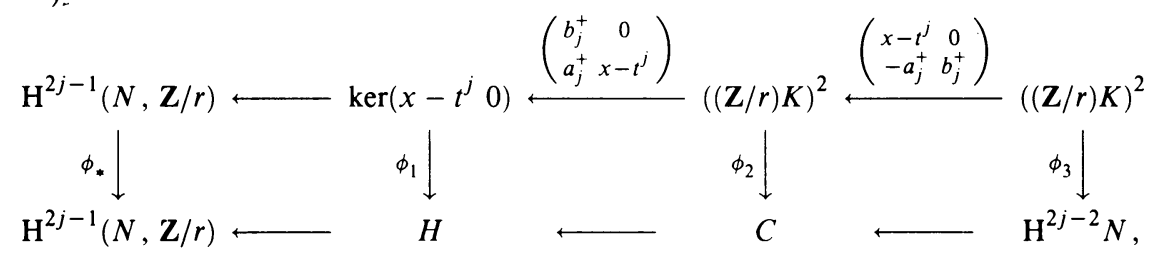

where we have written

$$
H=\operatorname{Hom}_{(\mathbf{Z} / r) N}\left(M_{2 j-1} \otimes(\mathbf{Z} / r), \mathbf{Z} / r\right), \quad C=\left(\operatorname{coker}\left(d^{2 j-2}\right)\right) \otimes(\mathbf{Z} / r)
$$

for short and where $\phi_{*}$ denotes the induced map on cohomology. We claim that $\phi_{*}$ is necessarily the identity map. In fact, the top row of (5.5.4) may be 
viewed as the beginning of a free resolution of $\mathrm{H}^{2 j} N$ in the category of abelian groups, and so may the bottom row, when we discard the kernel $\mathrm{H}^{2 j-2} N$. The standard comparison argument implies that $\phi_{*}$ is an isomorphism, and we may assume things have been arranged in such a way that $\phi_{*}$ is the identity map.

The embedding $\mathrm{s}(5.3 .5) \longrightarrow(\mathrm{Z} / r) \otimes(5.3 .1)$ explained in subsection 5.3 (cf. (5.3.7)), factors through the top row of (5.5.5), and hence combining (5.5.5) with this embedding yields a solution of the extension problem

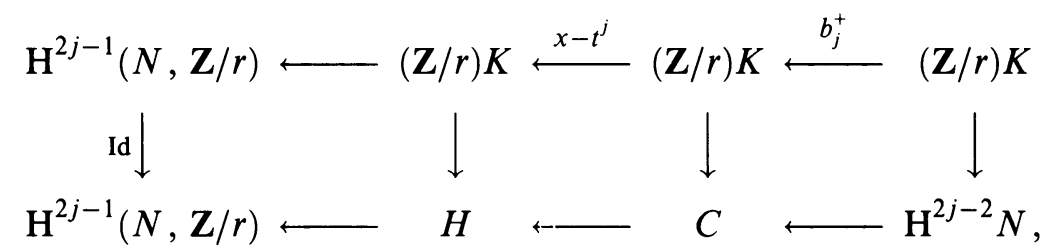

which is now entirely phrased over $\mathbf{Z} / r$ as ground ring; for completeness we mention that, as before,

$$
H=\operatorname{Hom}_{(\mathbf{Z} / r) N}\left(M_{2 j-1} \otimes(\mathbf{Z} / r), \mathbf{Z} / r\right), \quad C=\left(\operatorname{coker}\left(d^{2 j-2}\right)\right) \otimes(\mathbf{Z} / r) .
$$

Hence the solution of this extension problem yields the class

$$
v_{2}^{2 j-1}\left(\mathbf{e}_{\nu}, \mathbf{Z} / r\right) \in \operatorname{Ext}_{(\mathbf{Z} / r) K}^{2}\left(\mathrm{H}^{2 j-1}(N, \mathbf{Z} / r), \mathrm{H}^{2 j-2}(N, \mathbf{Z} / r)\right) \cong \mathbf{Z} / q_{1} .
$$

To compute the class $v_{2}^{1}\left(\mathbf{e}_{\nu}, \mathbf{Z} / r\right)$, define a morphism

$$
\Phi: I G \otimes_{\mathbf{Z N}}(\mathbf{Z} / r) \longrightarrow \mathbf{Z} / r
$$

of $(\mathbf{Z} / r)$-modules by means of the formulas $\Phi(y-1)=1$ and

$$
\Phi(x-1) x^{l}= \begin{cases}f, & \text { if } l=0, \\ 0, & \text { if } 1 \leq l \leq s-1 .\end{cases}
$$

Henceforth we use the convention $(\Phi)(1)=\Phi$, etc. Inspection shows that the diagram

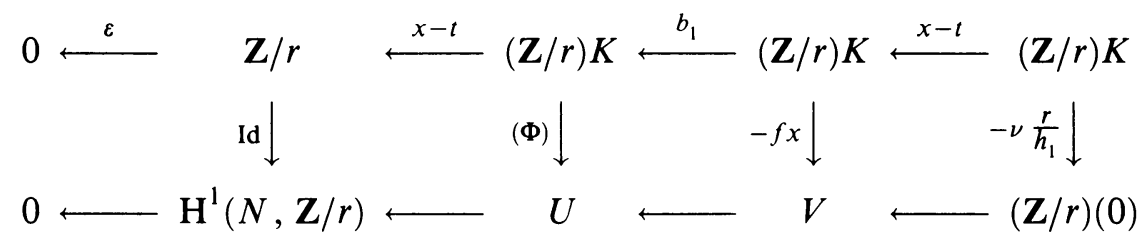

is commutative, where we have written

$$
U=\operatorname{Hom}\left(I G \otimes_{\mathbf{Z N}}(\mathbf{Z} / r), \mathbf{Z} / r\right), \quad V=\operatorname{Hom}((\mathbf{Z} / r) K, \mathbf{Z} / r)
$$

for short. By virtue of $[26,(2.5)]$ we conclude that

$$
v_{2}^{1}\left(\mathbf{e}_{\nu}, \mathbf{Z} / r\right)=-\nu\left[\frac{r}{h_{1}}\right] \in \operatorname{Ext}_{(\mathbf{Z} / r) K}^{2}\left(\mathrm{H}^{1}(N, \mathbf{Z} / r), \mathrm{H}^{0}(N, \mathbf{Z} / r)\right) .
$$

In view of (5.3.9(1)) this implies assertion (1) of the theorem.

For $j \geq 2$, an argument of this kind cannot yield a complete description of the classes $v_{3}^{2 j}$ since the corresponding morphism $\kappa$ (occurring in (5.3.10) 
above) is no longer injective. Moreover, for $j \geq 2$, the recipe in (2.5) of [26] leads to a mess. We therefore proceed otherwise:

The obvious chain map

$$
\begin{aligned}
\operatorname{Hom}_{\mathbf{Z} N}(\mathbf{P}, \mathbf{Z}) & \longrightarrow \operatorname{Hom}_{\mathbf{Z} N}(\mathbf{P}, \operatorname{Hom}(\mathbf{Q} / \mathbf{Z}, \mathbf{Q} / \mathbf{Z})) \\
& \cong \operatorname{Hom}\left(\mathbf{P} \otimes_{\mathbf{Z N}}(\mathbf{Q} / \mathbf{Z}), \mathbf{Q} / \mathbf{Z}\right)
\end{aligned}
$$

is compatible with the obvious $\mathbf{Z} K$-module structures, and, for $j \geq 0$, induces morphisms

$$
\mathbf{H}^{j}(N) \longrightarrow \mathbf{H}^{j}\left(\operatorname{Hom}\left(\mathbf{P} \otimes_{\mathbf{Z N}}(\mathbf{Q} / \mathbf{Z}), \mathbf{Q} / \mathbf{Z}\right)\right)=\operatorname{Hom}\left(\mathbf{H}_{j}(N, \mathbf{Q} / \mathbf{Z}), \mathbf{Q} / \mathbf{Z}\right)
$$

of $(\mathbf{Z} K)$-modules. Since the group $N$ is finite, for $j \geq 1$ these are in fact isomorphisms. Consequently we can compute the characteristic classes $v_{3}^{2 j}$ from the chain complex

$$
\operatorname{Hom}\left(\mathbf{P} \otimes_{\mathbf{Z N}}(\mathbf{Q} / \mathbf{Z}), \mathbf{Q} / \mathbf{Z}\right)
$$

More precisely, application of the functor $\operatorname{Hom}(-, \mathbf{Q} / \mathbf{Z})$ to a 3-fold extension representing the class $w_{3}^{2 j}\left(\mathbf{e}_{\nu}, \mathbf{Q} / \mathbf{Z}\right)$ yields a 3-fold extension representing the class $v_{3}^{2 j+2}$. To compute its value in $\operatorname{Ext}_{\mathrm{ZK} K}^{3}\left(\mathrm{H}^{2 j+2} N, \mathrm{H}^{2 j} N\right)$, we take (5.4.14) as a representative of $w_{3}^{2 j}\left(\mathbf{e}_{\nu}, \mathbf{Q} / \mathbf{Z}\right)$ and proceed as follows:

Consider the chain complex

$$
0 \leftarrow(\mathbf{Z} / r)(-j) \stackrel{\varepsilon}{\longleftarrow}(\mathbf{Z} / r) K \stackrel{t^{j} x-1}{\longleftarrow}(\mathbf{Z} / r) K \stackrel{b_{j}^{-}}{\longleftarrow} \mathbf{Z} K \stackrel{\left(t^{j} x-1 \quad r\right)}{\longleftarrow}(\mathbf{Z} K)^{2}
$$

which already occurred in (5.4.10). By our conventions it is a chain complex in the category of right $(\mathbf{Z K})$-modules. A little thought reveals that its dual $\operatorname{Hom}((5.5 .7), \mathbf{Q} / \mathbf{Z})$ in the category of right $(\mathbf{Z} K)$-modules may be written

$$
\begin{aligned}
\operatorname{Hom}\left((\mathbf{Z} K)^{2}, \mathbf{Q} / \mathbf{Z}\right) \stackrel{\stackrel{\left(t^{j} x-1, r\right)^{*}}{\longleftarrow} \operatorname{Hom}(\mathbf{Z} K, \mathbf{Q} / \mathbf{Z})}{\stackrel{\Omega}{\longleftarrow} R K \stackrel{t^{j} x^{-1}-1}{\longleftarrow} R K \stackrel{t^{j} b_{j}^{+}}{\longleftarrow} R(j) \leftarrow 0,}
\end{aligned}
$$

where we have written $R=\mathbf{Z} / r$ for short and where $\Omega$ is the obvious morphism of right $(\mathbf{Z} K)$-modules, so that $(\Omega(1))(1)=\frac{1}{r}$; here we use the identification

$$
(\mathbf{Z} / r) K \stackrel{\cong}{\longrightarrow} \operatorname{Hom}((\mathbf{Z} / r) K, \mathbf{Q} / \mathbf{Z}), \quad 1 \mapsto \omega:(\mathbf{Z} / r) K \rightarrow \mathbf{Q} / \mathbf{Z},
$$

as right $(\mathbf{Z} K)$-modules where, for $0 \leq l \leq s-1$,

$$
\omega\left(x^{l}\right)=\delta_{0, l} / r \quad(\text { Kronecker } \delta) .
$$

Next we recall the cocycle

$$
\left(\frac{t^{j}-1}{h_{1}}, \frac{r}{h_{1}}\right):(\mathbf{Z} K)^{2} \longrightarrow(\mathbf{Z} / r)(-j-1)
$$

given in $(5.3 .4(3))$ representing the class $\vartheta_{3}^{j}$. It is clear that its composite $\frac{1}{r}\left(\frac{t^{j}-1}{h_{1}}, \frac{r}{h_{1}}\right)$ with the obvious injection into $\mathbf{Q} / \mathbf{Z}$, viewed as an element of 
$\operatorname{Hom}\left((\mathbf{Z} K)^{2}, \mathbf{Q} / \mathbf{Z}\right)$, has a preimage in $\operatorname{Hom}(\mathbf{Z} K, \mathbf{Q} / \mathbf{Z})$, i.e., that the extension problem

$$
\begin{aligned}
& \mathbf{Z} K \stackrel{\left(t^{j} x-1, r\right)}{\longleftarrow} \quad(\mathbf{Z} K)^{2} \\
& \Phi\left\lfloor\quad\left(\frac{t^{j}-1}{h_{1}}, \frac{r}{h_{1}}\right)\right\rfloor \\
& \mathbf{Q} / \mathbf{Z} \underset{\frac{1}{r}}{\longleftarrow}(\mathbf{Z} / r)(-j-1)
\end{aligned}
$$

has a solution $\Phi$. For example, for $0 \leq l \leq s-1$, let

$$
\Phi\left(x^{l}\right)=\frac{1}{t^{(j+1) l} r h_{1}}+\frac{\mathrm{B}\left(x^{l}\right)}{r},
$$

where $\mathbf{B}: \mathbf{Z} K \longrightarrow \mathbf{Q} / \mathbf{Z}$ is a morphism with $\mathbf{B}(1)=0$, and which is determined by

$$
\Phi\left(\left(t^{j} x-1\right) x^{l}\right)=\frac{1}{r} \frac{t^{j}-1}{h_{1}} t^{-l(j+1)} \in \mathbf{Q} / \mathbf{Z}, \quad 0 \leq l \leq s-1 .
$$

For completeness, we mention that this amounts to

$$
\frac{t^{j}-1}{t^{(j+1) l} h_{1}}=\frac{t^{s-1}-1}{t^{(j+1) l} h_{1}}+t^{j} \mathrm{~B}\left(x^{(l+1)}\right)-\mathrm{B}\left(x^{l}\right), \quad 0 \leq l \leq s-2,
$$

but we shall not need an explicit formula for B.

We can now compute the class $v_{3}^{2 j+2}$ : Let $\mathbf{P}_{G}(\mathbf{Z})=\mathbf{M}(G)$ and let

$$
\begin{aligned}
\Theta_{3}^{j}=\left(\frac{t^{j}-1}{h_{1}}, \frac{r}{h_{1}}\right)^{*}:(\mathbf{Z} / r)(j+1)= & \operatorname{Hom}((\mathbf{Z} / r)(-j-1), \mathbf{Q} / \mathbf{Z}) \\
& \longrightarrow \operatorname{Hom}\left((\mathbf{Z} K)^{2}, \mathbf{Q} / \mathbf{Z}\right)
\end{aligned}
$$

be the dual of the above cocycle representing the class $\vartheta_{3}^{j}$, as indicated.

Lemma 5.5.10. Let $j \geq 0$, and let

$$
\begin{aligned}
\lambda_{j}=\frac{1-t^{j+1}}{h_{j}}\left(t^{(j+1)(s-2)}+t^{(j+1)(s-3)}\left(x+t^{j}\right)\right. & \\
& \left.+\cdots+\left(x^{s-2}+t^{j} x^{s-3}+t^{2 j} x^{s-4}+\cdots+t^{(s-2) j}\right)\right) .
\end{aligned}
$$


Then

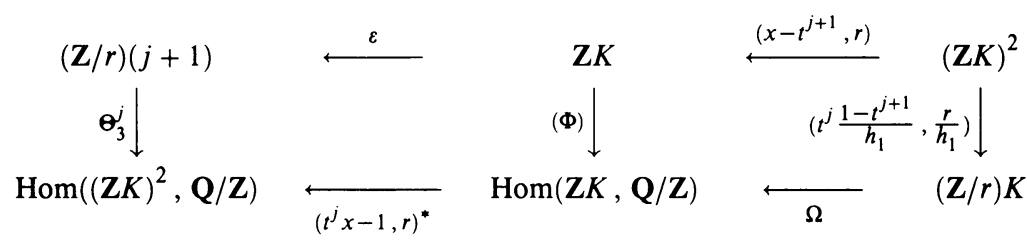

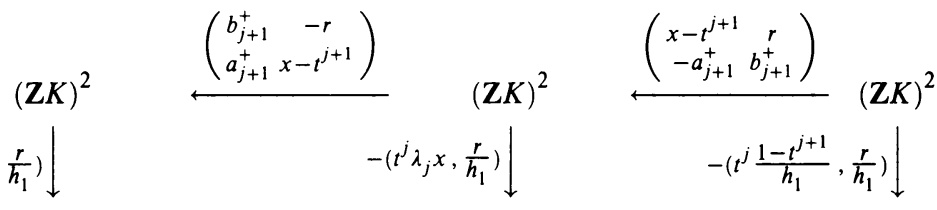

$$
\begin{aligned}
& (\mathbf{Z} / r) K \\
& \overleftarrow{t^{j} x^{-1}-1} \quad(\mathbf{Z} / r) K
\end{aligned}
$$

is a commutative diagram in the category of right $(\mathbf{Z K})$-modules; here the top row is the free resolution (5.3.1) of $(\mathbf{Z} / r)(j+1)$, and (for typographical reasons) the diagram has been split into two halves so that the middle vertical arrow appears twice.

Proof. At first we indicate an argument for the commutativity of the diagram

$$
\begin{array}{ccccc}
(\mathbf{Z} / r)(j+1) & \stackrel{\varepsilon}{\mathbf{Z} K} & \stackrel{\left(x-t^{j+1}, r\right)}{(\mathbf{Z} K)^{2}} & (\mathbf{\Phi}) \downarrow & \left(t^{j} \frac{1-t^{j+1}}{h_{1}}, \frac{r}{h_{1}}\right) \downarrow \\
\mathbf{\Theta}_{3}^{j} \downarrow & & \longleftarrow & \\
\operatorname{Hom}\left((\mathbf{Z} K)^{2}, \mathbf{Q} / \mathbf{Z}\right) & \stackrel{\mathbf{Z} / r)(j)=\operatorname{ker}(\mathbf{\Omega}),}{\varepsilon_{\left(t^{j} x-1, r\right)^{*}}} \operatorname{Hom}(\mathbf{Z K}, \mathbf{Q} / \mathbf{Z}) & \stackrel{\longleftarrow}{\varepsilon^{*}}
\end{array}
$$

where $(\mathbf{Z} / r)(j)$ is identified with $\operatorname{Hom}((\mathbf{Z} / r)(-j), \mathbf{Q} / \mathbf{Z})$ in the obvious way: By construction, $(\Phi)(r)=r \Phi$, and in view of the definition of $\Phi$ this induces the morphism $(\mathbf{Z} / r)(-j) \rightarrow \mathbf{Q} / \mathbf{Z}$ sending 1 to $\frac{1}{h_{1}}$, which under the identification of $\operatorname{Hom}((\mathbf{Z} / r)(-j), \mathbf{Q} / \mathbf{Z})$ with $(\mathbf{Z} / r)(j)$ corresponds to $\frac{r}{h_{1}} \in(\mathbf{Z} / r)(j)$. Likewise,

$$
(\Phi)\left(x-t^{j+1}\right)(1)=\Phi\left(x^{s-1}\right)-\Phi\left(t^{j+1}\right) .
$$

However, (5.5.9) with $l=s-1$ reads

$$
\Phi\left(\left(t^{j} x-1\right) x^{s-1}\right)=\frac{t^{j}-1}{h_{1}} t^{j+1} \in(\mathbf{Z} / r)(-j-1),
$$

and we conclude that

$$
\frac{t^{j}}{r h_{1}}-\Phi\left(x^{s-1}\right)=\frac{t^{j}-1}{r h_{1}} t^{j+1}
$$

whence

$$
\Phi\left(x^{s-1}\right)-\Phi\left(t^{j+1}\right)=\frac{t^{j}}{r h_{1}}-\frac{t^{j}-1}{r h_{1}} t^{j+1}-\frac{t^{j+1}}{r h_{1}}=t^{j} \frac{1-t^{j+1}}{r h_{1}}
$$

as desired. 
The rest of the proof now comes down to

$$
\begin{gathered}
\frac{1-t^{j+1}}{h_{1}} b_{j+1}^{+}+\frac{r}{h_{1}} a_{j+1}^{+}=\lambda_{j}\left(x-t^{j}\right), \quad \frac{r}{h_{1}} b_{j+1}^{+}=\frac{r}{h_{1}} b_{j}^{+}, \\
\lambda_{j}\left(x-t^{j+1}\right)-\frac{r}{h_{1}} a_{j+1}^{+}=\frac{1-t^{j+1}}{h_{1}} b_{j}^{+} .
\end{gathered}
$$

We leave the details to the reader.

\section{THE PROOF OF THEOREM 0.4 AND THE COLLAPSING OF THE SPECTRAL SEQUENCE AT A GOOD PRIME}

We now have the machinery in place to compute the first nonzero differential in the cohomology spectral sequence $\left(E_{i}^{*, *}\left(\mathbf{e}_{\nu}, \mathbf{Z}\right), d_{i}\right)$ of the group extension $\mathbf{e}_{\nu}$. The corresponding graded group $E_{2}$ is the same as that given in $\S 2$ above, and for degree reasons the differential $d_{2}$ is zero. Hence the spectral sequence has $E_{2}=E_{3}$ and the first nonzero differential is $d_{3}$. A description thereof has been given in Theorem 0.4 in the Introduction. We now prove Theorem 0.4 .

It is not hard to see (cf. (6.8) below) that, for $j \geq 0$, the numbers $q_{j}$ are divisible by $q_{1}$. For convenience, we shall henceforth write

$$
A_{j}=A_{j}(s, t)=j\left(1+t^{j-1}+\cdots+t^{(s-1)(j-1)}\right)-(j-1)\left(1+t^{j}+\cdots+t^{(s-1) j}\right) .
$$

Here is the crucial step in the proof.

Lemma 6.1. The differential $d_{3}: E_{3}^{k, 2 j} \longrightarrow E_{3}^{k+3,2 j-2}$, where $k \geq 0$ and $j \geq 1$, in the spectral sequence $\left(E_{i}^{*, *}\left(\mathbf{e}_{\nu}, \mathbf{Z}\right), d_{i}\right)$ of the group extension $\mathbf{e}_{\nu}$ is zero for $k=2 i$, while for $k=2 i+1$,

$$
d_{3}\left(\frac{r}{k_{j}} c_{x}^{i} \omega_{x} c_{y}^{j}\right)=-\nu \frac{h_{j-1} A_{j}}{h_{1} k_{j}}\left(\frac{r}{h_{j-1}} c_{x}^{i+2} c_{y}^{j-1}\right) .
$$

Proof. By (2.4) of [26], the differential $d_{3}$ of the cohomology spectral sequence is given by Yoneda pairing with the class $v_{3}^{2 j}$. In view of (2.5) of [26], the result $z \cdot v_{3}^{2 j}$ of Yoneda pairing a class $z \in \mathrm{H}^{k}\left(K, \mathrm{H}^{2 j} N\right)$ with $v_{3}^{2 j}$ can be computed as follows: Take the free resolution (5.3.1) of $\mathrm{H}^{2 j} N$ and the standard one (say) $\mathbf{P}_{\mathbf{Z} K}(\mathbf{Z})$ of $\mathbf{Z}$ (reproduced in $\S 5.1$ above) in the category of right $\mathbf{Z} K$-modules, and lift a $k$-cocycle for $z$ to a cycle $\zeta_{z}$ in $\operatorname{Hom}_{\mathbf{Z} K}\left(\mathbf{P}_{\mathbf{Z K}}(\mathbf{Z}),(5.3 .1)\right)$ of degree $-k$ so that under the obvious morphism

$$
\varepsilon_{*}: \operatorname{Hom}_{\mathbf{Z K}}\left(\mathbf{P}_{\mathbf{Z K}}(\mathbf{Z}),(5.3 .1)\right) \longrightarrow \operatorname{Hom}_{\mathbf{Z K}}\left(\mathbf{P}_{\mathbf{Z K}}(\mathbf{Z}), \mathrm{H}^{2 j} N\right)
$$

$\zeta_{z}$ goes to $z$. Then the composite of $\zeta_{z}$ with a cocycle for $v_{3}^{2 j}$ is a cocycle for $z \cdot v_{3}^{2 j}$. We shall take $z$ to be the generator of $E_{2}^{k, 2 j}=E_{3}^{k, 2 j}$ given in (6.1) above; we shall carry out this procedure now, distinguishing the two cases $k$ even and $k$ odd. 
Let $k=2 i$, and let $j \geq 1$. We wish to compute $\left(\frac{r}{h_{j}} c_{x}^{i} c_{y}^{j}\right) \cdot \tau_{3}^{j}$. To this end, let

$$
\delta_{j}=\frac{t^{j(s-1)}-1}{h_{j}}+\frac{t^{j(s-2)}-1}{h_{j}} x+\cdots+\frac{t^{j}-1}{h_{j}} x^{s-2} \in \mathbf{Z K} .
$$

Then a straightforward calculation shows that the diagram

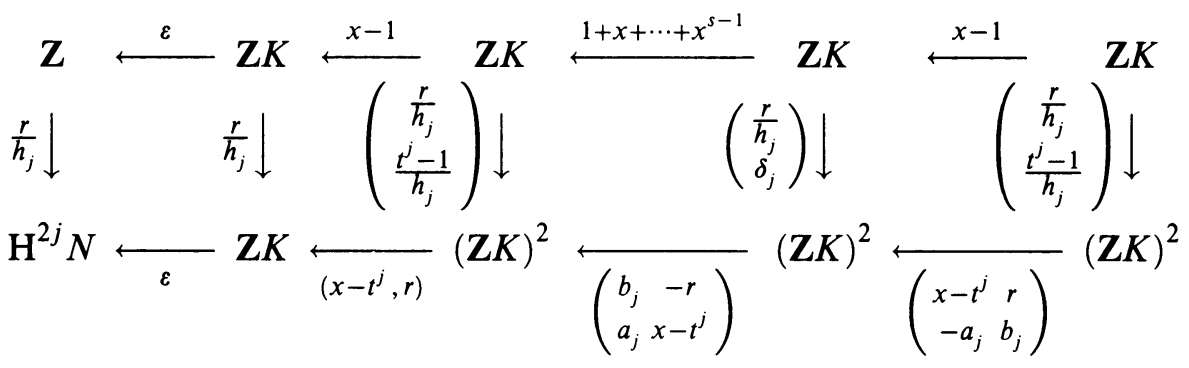

is commutative. It displays a lifting of $\frac{r}{h_{j}} c_{x}^{i} c_{y}^{j}$ to a cycle in

$$
\operatorname{Hom}_{\mathbf{Z K}}\left(\mathbf{P}_{\mathbf{Z K}}(\mathbf{Z}),(\text { 5.3.1)). }\right.
$$

As indicated above, for any right $\mathbf{Z} K$-module $U$, it may be used to compute the Yoneda pairing

$$
\because \mathrm{H}^{2 i}\left(K, \mathrm{H}^{2 j} N\right) \otimes \mathrm{Ext}_{\mathrm{ZK}}^{*}\left(\mathrm{H}^{2 j} N, U\right) \longrightarrow \mathrm{H}^{2 i+*}(K, U) .
$$

In particular, we may take $U=\mathrm{H}^{2 j-2} N,^{*}=3$, and (cf. (5.3.4)) the cocycle $\left(\frac{1-t^{j}}{h_{1}}, \frac{r}{h_{1}}\right)$ of $(5.3 .1)$ as a representative of $\tau_{3}^{j}$ and conclude

$$
\left(\frac{r}{h_{j}} c_{x}^{i} c_{y}^{j}\right) \cdot \tau_{3}^{j}=\frac{r}{h_{1} h_{j}}\left(1-t^{j}+t^{j}-1\right) c_{x}^{i+1} \omega_{x} c_{y}^{j-1}=0 \in E_{3}^{2 i+3,2 j-2} .
$$

However, by virtue of $(5.5 .1), v_{3}^{2 j}=-\nu \tau_{3}^{j}$, whence, in view of $(2.1)$, the corresponding differential is zero as asserted.

Next, let $k=2 i+1$ and let $j \geq 1$. We wish to compute $\left(\frac{r}{k_{j}} c_{x}^{i} \omega_{x} c_{y}^{j}\right) \cdot \tau_{3}^{j}$. To this end, let

$$
\begin{aligned}
\sigma_{j}(z)= & \frac{r}{k_{j}} \sum_{0 \leq \nu+\mu \leq s-2} t^{\nu j} z^{\mu} \in \mathbf{Z}[z] \\
= & \frac{r}{k_{j}}\left(1+z+t^{j}+z^{2}+t^{j} z+t^{2 j}+\cdots\right. \\
& \left.\quad+z^{s-2}+t^{j} z^{s-3}+t^{2 j} z^{s-4}+\cdots+t^{(s-2) j}\right), \\
\alpha_{j}= & \frac{1+t^{j}+\cdots+t^{(s-1) j}}{k_{j}} .
\end{aligned}
$$

Then it is clear that

$$
\left(z-t^{j}\right) \sigma_{j}=\frac{r}{k_{j}}\left(1+z+\cdots+z^{s-1}\right)-r \alpha_{j} \in \mathbf{Z}[z] .
$$


Let $\sigma_{j}=\sigma_{j}(x) \in \mathbf{Z} K \cong \mathbf{Z}[z] /\left(z^{s}-1\right)$. Then (6.2) and a straightforward calculation show that the diagram

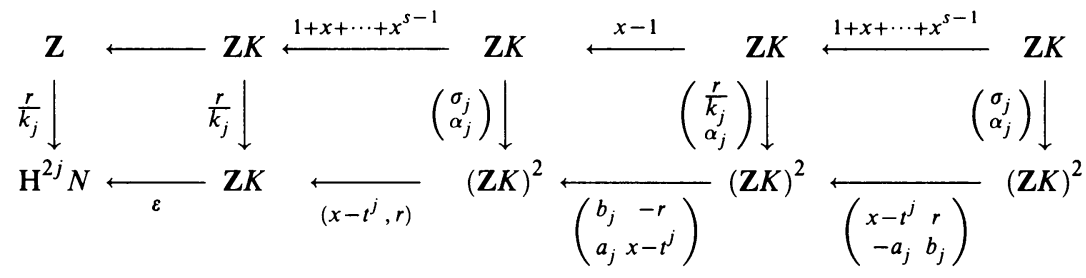

is commutative. It displays a lifting of $\frac{r}{k_{j}} c_{x}^{i} \omega_{x} c_{y}^{j}$ to a cycle in

$$
\operatorname{Hom}_{\mathbf{Z K}}\left(\mathbf{P}_{\mathbf{Z K}}(\mathbf{Z}),(\text { 5.3.1)). }\right.
$$

As indicated above, for any right $\mathbf{Z} K$-module $U$, it may be used to compute the Yoneda pairing

$$
\cdot: \mathrm{H}^{2 i+1}\left(K, \mathrm{H}^{2 j} N\right) \otimes \operatorname{Ext}_{\mathrm{Z} K}^{*}\left(\mathrm{H}^{2 j} N, U\right) \longrightarrow \mathrm{H}^{2 i+1+*}(K, U) .
$$

In particular, we may take $U=\mathrm{H}^{2 j-2} N,{ }^{*}=3$, and (cf. (5.3.4)) the cocycle $\left(j t^{j-1} \frac{1-t}{h_{1}}, \frac{r}{h_{1}}\right)$ of (5.3.1) as a representative of $\tau_{3}^{j}$ and conclude

$$
\begin{aligned}
\left(\frac{r}{k_{j}} c_{x}^{i} \omega_{x} c_{y}^{j}\right) \cdot \tau_{3}^{j} & =\left(j t^{j-1} \frac{(1-t)}{h_{1}} \sigma_{j}+\frac{r}{h_{1}} \alpha_{j}\right) c_{x}^{i+2} c_{y}^{j-1} \\
& \in E_{3}^{2 i+4,2 j-2} \cong \mathbf{Z} / q_{j-1} .
\end{aligned}
$$

To obtain the formula asserted in the lemma, we specialize (6.2) with $z=t^{j-1}$, i.e., we pass from $\mathbf{Z}[z]$ to $\mathbf{Z}[z] /\left(z-t^{j-1}\right) \cong \mathbf{Z}$ and conclude that, for $j \geq 1$,

$$
\begin{aligned}
& \left(t^{j-1}-t^{j}\right) \sigma_{j}\left(t^{j-1}\right) \\
& \quad=\frac{r}{k_{j}}\left(1+t^{j-1}+\cdots+t^{(s-1)(j-1)}-\left(1+t^{j}+\cdots+t^{(s-1) j}\right)\right) \in \mathbf{Z},
\end{aligned}
$$

whence

$$
\left(j t^{j-1} \frac{(1-t)}{h_{1}} \sigma_{j}\left(t^{j-1}\right)+\frac{r}{h_{1}} \alpha_{j}\right)=\frac{r A_{j}}{h_{1} k_{j}} \in \mathbf{Z}
$$

Since $\mathrm{H}^{2 j-2} N=(\mathbf{Z} / r)(j-1)\left\langle c_{y}^{j-1}\right\rangle$, we conclude

$$
c_{y}^{j-1}\left(j t^{j-1} \frac{(1-t)}{h_{1}} \sigma_{j}+\frac{r}{h_{1}} \alpha_{j}\right)=\frac{r A_{j}}{h_{1} k_{j}} c_{y}^{j-1} \in \mathrm{H}^{2 j-2} N .
$$

However, by virtue of (5.5.1), $v_{3}^{2 j}=-\nu \tau_{3}^{j}$, whence, in view of (6.1), the corresponding differential is given by the formula in the lemma.

As before, for a prime $p$ and any number $l$ we denote the $p$-part of $l$ by $l_{p}$. Our next aim is to deduce Theorem 0.4 from (6.1). To this end we elaborate on the number theory in $\S 3$ above to have available appropriate tools so that we can prove Theorem 0.4 which is an integral statement. 
Lemma 6.6. Let $p$ be a prime, let $t$ be a number so that $t \equiv 1 \bmod p$, and let $\alpha$ be an arbitrary number.

(1) If $p$ is odd or $p=2$ and $t \equiv 1 \bmod 4$, for some $\beta, \frac{t^{p^{\alpha}}-1}{t-1}=p^{\alpha}(1+\beta p)$.

(2) If $p=2$ and $t \equiv-1 \bmod 4,\left(\frac{t^{2^{\alpha}}-1}{t-1}\right)_{2}=2^{\alpha-1}(t+1)_{2}$.

Proof. See Lemma 2.2 in [26].

Next we recall that the number $q_{1}=\frac{h_{1} k_{1}}{r}$ is the order of $\mathrm{H}^{2}(K, N)$. We now assume that $q_{1}>1$ and pick a prime $p$ dividing this number. Since $q_{1}$ divides the number $h_{1}=(t-1, r), p$ divides $h_{1}$ as well, whence $t \equiv 1 \bmod p$. Moreover, it is manifest that $p$ divides $r$ and $s$.

Lemma 6.7. Let $p$ be a prime which divides $q_{1}$. Then the following are true.

(1) For $j \geq 1,\left(A_{j}\right)_{p}=s_{p}$.

(2) If $p$ is odd or $p=2$ and $t \equiv 1 \bmod 4$,

$$
\begin{aligned}
& \left(h_{j}\right)_{p}=\left(h_{1}\right)_{p}\left(j_{p}, \frac{r}{h_{1}}\right)=\left(h_{1}\right)_{p}\left(j, d_{p}\right), \\
& \left(k_{j}\right)_{p}=(r, s)_{p}, \\
& \left(q_{j}\right)_{p}=\left(\frac{h_{1}(r, s)}{r}\right)_{p}\left(j_{p}, \frac{r}{h_{1}}\right)=\left(q_{1}\right)_{p}\left(j_{p}, \frac{r}{h_{1}}\right)=\left(q_{1}\right)_{p}\left(j, d_{p}\right) .
\end{aligned}
$$

(3) If $p=2$ and $t \equiv-1 \bmod 4$,

$$
\begin{aligned}
& \left(h_{j}\right)_{2}= \begin{cases}(t+1)_{2}\left(j_{2}, \frac{r}{(t+1, r)}\right)=(t+1)_{2}\left(j, d_{2}\right), & \text { if } j \text { is even, } \\
2, & \text { if } j \text { is odd, }\end{cases} \\
& \left(k_{j}\right)_{2}= \begin{cases}(r, s)_{2}, & \text { if } j \text { is even, } \\
\begin{cases}\frac{r_{2}}{2}, & \text { if } \frac{s(t+1)}{r} \text { and } j \text { are both odd, } \\
r_{2}, & \text { if } \frac{s(t+1)}{r} \text { is even while } j \text { is odd, }\end{cases} \end{cases}
\end{aligned}
$$

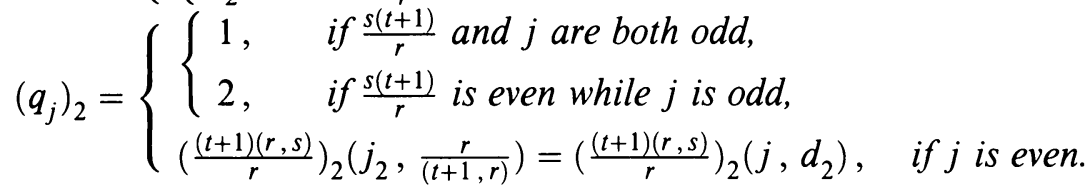

Corollary 6.8. For $j \geq 2$, the numbers $q_{j}$ are divisible by $q_{1}$.

Proof of 6.7. If $t=1$, there is nothing to prove, and we assume $t>1$. For $j \geq 1$, we may then write

$$
A_{j}=j \frac{t^{(j-1) s}-1}{t^{j-1}-1}-(j-1) \frac{t^{j s}-1}{t^{j}-1} .
$$

With $s=s_{p} s^{\prime}$, where $\left(s^{\prime}, p\right)=1$ and $t^{\prime}=t^{s^{\prime}}$, this may be rewritten as

$$
\begin{aligned}
A_{j}= & \left(j \frac{t^{(j-1) s_{p}}-1}{t^{\prime j-1}-1}-(j-1) \frac{t^{j s_{p}}-1}{t^{\prime j}-1}\right) \frac{t^{(j-1)}-1}{t^{j-1}-1} \\
& +(j-1) \frac{t^{\prime j s_{p}}-1}{t^{\prime j}-1}\left(\frac{t^{(j-1) s^{\prime}}-1}{t^{j-1}-1}-\frac{t^{j s^{\prime}}-1}{t^{j}-1}\right) .
\end{aligned}
$$


Furthermore, since $t \equiv 1 \bmod p$,

$$
\frac{t^{(j-1) s^{\prime}}-1}{t^{j-1}-1}-\frac{t^{j s^{\prime}}-1}{t^{j}-1} \equiv 0 \bmod p
$$

and, in view of $(3.1), \frac{t^{\prime j s_{p}}-1}{t^{\prime j}-1}$ is divisible by $s_{p}$ while $\frac{t^{(j-1)}-1}{t^{j-1}-1}$ is prime to $p$. Hence it suffices to show that

$$
A_{j}^{\prime}=j \frac{t^{(j-1) s_{p}}-1}{t^{\prime j-1}-1}-(j-1) \frac{t^{\prime j s_{p}}-1}{t^{\prime j}-1}
$$

is divisible by $s_{p}$ exactly. If $p$ is odd or $p=2$ and $t \equiv 1 \bmod 4$, we employ $(6.6)(1)$ and rewrite $A_{j}^{\prime}$ in the form

$$
A_{j}^{\prime}=j s_{p}\left(1+p \beta_{j-1}\right)-(j-1) s_{p}\left(1+p \beta_{j}\right) .
$$

It is then clear that $A_{j}^{\prime}$ is divisible by $s_{p}$ exactly. If $p=2$ and $t \equiv-1 \bmod 4$, we exploit $(6.6)(2)$ and conclude that

$$
\begin{gathered}
\left(\frac{t^{(j-1) s_{p}}-1}{t^{\prime j-1}-1}\right)_{2} \begin{cases}=s_{2}, & \text { if } j \text { is odd, } \\
>s_{2}, & \text { if } j \text { is even, }\end{cases} \\
\left(\frac{t^{\prime j s_{p}}-1}{t^{\prime j}-1}\right)_{2} \begin{cases}>s_{2}, & \text { if } j \text { is odd, } \\
=s_{2}, & \text { if } j \text { is even, }\end{cases}
\end{gathered}
$$

whence $A_{j}^{\prime}$ is divisible by $s_{2}$ exactly. This proves (1).

The remaining statements follow at once from (3.1) (cf. also (3.2)). We only indicate that, e.g., if $p$ is odd or $p=2$ and $t \equiv 1 \bmod 4$,

$$
\begin{aligned}
\left(h_{j}\right)_{p} & =\left(\left(t^{j}-1\right)_{p}, r\right)=\left(j_{p}(t-1)_{p}, r\right) \\
& =\left(h_{1}\right)_{p}\left(j_{p}\left(\frac{t-1}{h_{1}}\right)_{p}, \frac{r}{h_{1}}\right)=\left(h_{1}\right)_{p}\left(j_{p}, \frac{r}{h_{1}}\right),
\end{aligned}
$$

since $\left(\frac{t-1}{h_{1}}\right)_{p}>1$ only if $\frac{r}{h_{1}}$ is not divisible by $p$.

Proof of Theorem 0.4. By (6.1), the differential $d_{3}: E_{3}^{k, 2 j} \longrightarrow E_{3}^{k+3,2 j-2}$ is zero for $k=2 i$, where $i \geq 0, j \geq 1$, while for $k=2 i+1$,

$$
d_{3}: E_{3}^{2 i+1,2 j} \cong \mathbf{Z} / q_{j} \longrightarrow \mathbf{Z} / q_{j-1} \cong E_{3}^{2 i+4,2 j-2}, \quad i \geq 0, j \geq 1,
$$

is given by

$$
d_{3}\left(\frac{r}{k_{j}} c_{x}^{i} \omega_{x} c_{y}^{j}\right)=-\nu \frac{h_{j-1} A_{j}}{h_{1} k_{j}}\left(\frac{r}{h_{j-1}} c_{x}^{i+2} c_{y}^{j-1}\right)
$$

Now

$$
\frac{h_{j-1} A_{j}}{h_{1} k_{j}}=\frac{q_{j-1}}{q_{1}} B_{j}, \quad \text { where } B_{j}=\frac{k_{1} A_{j}}{k_{j-1} k_{j}},
$$

and Lemma 6.7 implies that, at a prime $p$ dividing $q_{1}$, the numbers $B_{j}$ and $\frac{s}{(r, s)}$ coincide up to a unit. Furthermore, still in view of (6.7), at a prime which does not divide $q_{1}$, one of the two numbers $q_{j}$ and $q_{j-1}$ is a unit, 
whence either the domain or the range of the differential $d_{3}$ is zero. Hence the differential $d_{3}$ sends an appropriate generator to $-\nu \frac{q_{j-1}}{q_{1}} \frac{s}{(s, r)}$ times an appropriate generator.

For later reference we spell out the following.

Corollary 6.9. As a graded module over $\mathrm{H}^{*}(K, \mathbf{Z})=\left(\mathrm{P}\left[c_{x}\right]\right) / s c_{x}$, the object $E_{4}$ is generated by

$$
\begin{aligned}
c_{2 j} & =\frac{r}{h_{j}} c_{y}^{j} \in E_{4}^{0,2 j}, \quad j \geq 1, \\
x_{3} & =\frac{q_{1}}{\left(\nu, q_{1}\right)} \frac{r}{k_{1}} \omega_{x} c_{y} \in E_{4}^{1,2}, \\
x_{2 j+1} & =\frac{q_{1}}{\left(\nu \frac{s}{(r, s)}, q_{1}\right)} \frac{r}{k_{j}} \omega_{x} c_{y}^{j} \in E_{4}^{1,2 j}, \quad j \geq 2,
\end{aligned}
$$

subject to the relations

$$
\begin{aligned}
\frac{s}{q_{1}}\left(\nu, q_{1}\right) c_{x}^{i} & =0, & & i \geq 2, \\
h_{j} c_{2 j} & =0, & & j \geq 1, \\
q_{j} c_{x} c_{2 j} & =0, & & j \geq 1, \\
\frac{q_{j}}{q_{1}}\left(\nu \frac{s}{(r, s)}, q_{1}\right) c_{x}^{i} c_{2 j} & =0, & & i \geq 2, j \geq 1, \\
\left(\nu, q_{1}\right) x_{3} & =0, & & \\
\frac{q_{j}}{q_{1}}\left(\nu \frac{s}{(r, s)}, q_{1}\right) x_{2 j+1} & =0, & & \geq 2 .
\end{aligned}
$$

The proof is left to the reader.

Corollary 6.10. When $t \equiv-1 \bmod 4$ and the differential $d_{3}$ is nonzero at the prime 2 , the group $E_{4}^{k, l}(\mathbf{e}, \mathbf{Z})$ is nonzero at the prime 2 only if $l$ is even and $k=0,2$ or if $l$ is divisible by 4 .

Proof. In fact, by virtue of (6.7)(3), for $j$ odd, the number $q_{j}$ is at most divisible by 2 but not by 4 . Since the differential $d_{3}: E_{3}^{k, l} \rightarrow E_{3}^{k+3, l-2}$ is assumed nontrivial at the prime 2 , from (0.4) we conclude that groups of the kind $E_{3}^{*, 2 j}$ with $j$ odd survive to $E_{4}$ only if $*=0$ or $*=2$.

Theorem 6.11. The integral cohomology spectral sequence of e has $E_{4}=E_{\infty}$ at each prime $p$ so that $r_{p} \geq s_{p}$ or $f \equiv 0$ modulo $r_{p}$. Furthermore, as an algebra, $\mathrm{H}^{*}(G, \mathbf{Z})$ at $p$ is then as an algebra over $\mathrm{H}^{*}(K, \mathbf{Z})$ at $p$ generated by $\zeta_{2}, \xi_{3}, \zeta_{4}, \xi_{5}, \ldots, \zeta_{2 d_{p}}, \xi_{2 d_{p}+1}$.

Proof. By (4.22), for $j \geq 1$, the class $\operatorname{res}_{G \downarrow N}\left(\zeta_{2 j}\right)$ coincides with $c_{2 j}=\frac{r}{h_{j}} c_{y}^{j}$, perhaps up to a unit at $p$, and hence the class $c_{2 j}$ is an infinite cycle. Moreover, still by (4.22), the classes $\frac{q_{1}}{\left(\nu, q_{1}\right)} \frac{r}{k_{j}} \omega_{x} c_{y}^{j}$ are infinite cycles, too. Finally, keeping 
in mind that (2.1) describes $E_{2}$ for any extension of the kind (0.2), that is, for any extension of a finite cyclic group by a finite cyclic group, we see that, in view of (6.9), at a prime $p$ so that $r_{p} \geq s_{p}$, the classes $\frac{q_{1}}{\left(\nu, q_{1}\right)} \frac{r}{k_{j}} \omega_{x} c_{y}^{j}$ coincide with the classes $x_{2 j+1} \in E_{4}^{1,2 j}$, perhaps up to a unit at $p$.

Proposition 6.12. For a presentation (0.1) of an arbitrary metacyclic group $G$ and a prime $p$ so that $s_{p}>r_{p}$ the following are equivalent:

$$
\begin{aligned}
& f \not \equiv 0 \bmod r_{p}, \\
& \nu \not \equiv 0 \bmod \left(q_{1}\right)_{p} .
\end{aligned}
$$

Proof. Recall that $f$ may be written $f=\nu \frac{r}{h_{1}}$. Hence

$$
\nu \not \equiv 0 \bmod \left(q_{1}\right)_{p}=\left(\frac{h_{1} k_{1}}{r}\right)_{p}
$$

if and only if $f \not \equiv 0 \bmod \left(k_{1}\right)_{p}$. However, if $s_{p}>r_{p}$, in view of (6.7), the latter is equivalent to $f \not \equiv 0 \bmod r_{p}$. When $p$ is odd or $p=2$ and $t \equiv 1 \bmod 4$ this is immediate since then $\left(k_{1}\right)_{p}=r_{p}$, while when $p=2$ and $t \equiv-1 \bmod 4$, $\nu \not \equiv 0 \bmod \left(q_{1}\right)_{2}$ only if $\frac{s(t+1)}{r}$ is even and hence $\left(k_{1}\right)_{2}=r_{2}$.

We remind the reader that the notion of a prime that is good for the presentation $(0.1)$ has been introduced in the Introduction.

Corollary 6.13. The integral cohomology spectral sequence of e has $E_{4}=E_{\infty}$ at each prime $p$ that is good for the presentation (0.1).

Indeed, this follows at once from (2.15), (6.11), and (6.12).

\section{THE RESTRICTION OF THE SPECTRAL SEQUENCE TO $\mathrm{e}^{\prime}$}

In this section we examine the effect of restricting the spectral sequence to a suitable subgroup. We shall need this in the next section to solve the additive extension problem.

Let $G$ be an arbitrary metacyclic group given by a presentation of the kind (0.1), and as before we denote the corresponding group extension by $\mathbf{e}_{\nu}$, where $\nu=\frac{f(t-1, r)}{r}$. Recall that the number $q_{1}=\frac{h_{1} k_{1}}{r}$ is the order of $\mathrm{H}^{2}(K, N)$. We now assume that $q_{1}>1$, and we pick a prime $p$ dividing this number. As explained earlier, the prime $p$ divides $h_{1}$ as well, whence

$$
t \equiv 1 \bmod p,
$$

and $p$ divides $r$ and $s$, too. Extending terminology introduced in $\S 3$ for metacyclic $p$-groups we shall refer to the case where $p$ is odd or $p=2$ and $t \equiv 1 \bmod 4$ as the typical case and to the case where $p=2$ and $t \equiv-1 \bmod 4$ as the exceptional case. Notice that in view of (6.7)(3) the exceptional case occurs only if $\frac{(t+1) s}{r}$ is even. 
As before, let $G^{\prime}$ be the subgroup of $G$ generated by $y$ and $x^{\prime}=x^{p}$, with presentation

$$
G^{\prime}=G\left(r, s^{\prime}, t^{\prime}, f\right)=\left\langle x^{\prime}, y ; y^{r}=1, x^{s^{\prime}}=y^{f}, x^{\prime} y x^{\prime^{-1}}=y^{t^{\prime}}\right\rangle,
$$

where $s^{\prime}=\frac{s}{p}$ and $t^{\prime}=t^{p}$, and corresponding group extension $\mathrm{e}^{\prime}$ (cf. (4.2)), so that the injection maps yield a morphism

$$
(\mathrm{Id}, \cdot, \cdot): \mathbf{e}^{\prime} \longrightarrow \mathbf{e}
$$

of extensions. We mention in passing that in the typical case $s^{\prime}$ is still divisible by $p$ unless the $p$-Sylow subgroup of $G$ is abelian. In fact, since $q_{1}$ is divisible by $p$ so is $\frac{t^{s}-1}{r}$, whence, in view of (6.6), the number $\frac{s(t-1)}{r_{p}}$ is divisible by $p$; however, if $s_{p}=p$, the number $(t-1)_{p}$ is divisible by $r_{p}$ which can only happen if the $p$-Sylow subgroup of $G$ is abelian. On the other hand, in the exceptional case it may well happen that $s^{\prime}$ is an odd number while the 2Sylow subgroup of $G$ is nonabelian. For later reference we now describe the morphism on $E_{4}$ induced by (7.1).

The standard comparison argument entails the following.

\section{Proposition 7.2. A morphism}

Res: $E_{1}=\mathrm{P}\left[c_{x}\right] \otimes \Lambda\left[\omega_{x}\right] \otimes \mathrm{H}^{2 j}(N, \mathbf{Z}) \rightarrow E_{1}^{\prime}=\mathrm{P}\left[c_{x^{\prime}}\right] \otimes \Lambda\left[\omega_{x^{\prime}}\right] \otimes \mathrm{H}^{2 j}(N, \mathbf{Z})$ inducing the restriction map res: $E_{\geq 2}^{*, *}(\mathbf{e}, \mathbf{Z}) \longrightarrow E_{\geq 2}^{*, *}\left(\mathbf{e}^{\prime}, \mathbf{Z}\right)$ is given by

$$
\begin{aligned}
\operatorname{Res}\left(c_{x}^{i} \omega_{x} c_{y}^{j}\right) & =\left(1+\left(t^{j}\right)+\cdots+\left(t^{j}\right)^{p-1}\right) c_{x^{\prime}}^{i} \omega_{x^{\prime}} c_{y}^{j}, \\
\operatorname{Res}\left(c_{x}^{i} c_{y}^{j}\right) & =c_{x^{\prime}}^{i} c_{y}^{j} .
\end{aligned}
$$

Before we spell out the next proposition we remind the reader that a description of $E_{2}(\mathbf{e}, \mathbf{Z})$ has been given in $(2.1)$ and that $E_{2}(\mathbf{e}, \mathbf{Z})=E_{3}(\mathbf{e}, \mathbf{Z})$.

Proposition 7.3. For $i \geq 1$ and $j \geq 1$, the morphism (7.1) of extensions induces the commutative diagram

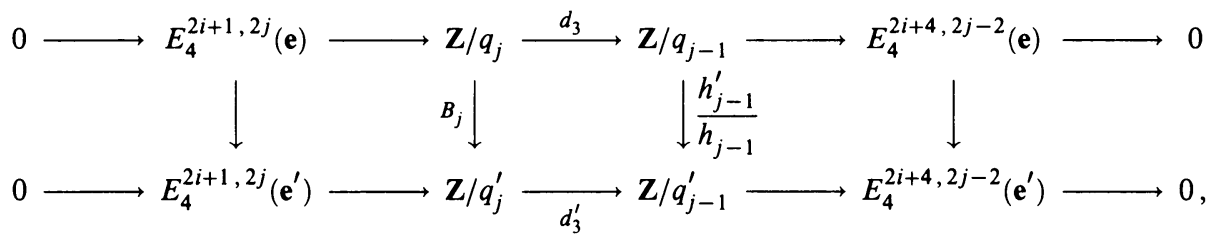

where we have written $E_{4}^{*, *}(\mathbf{e})=E_{4}^{*, *}(\mathbf{e}, \mathbf{Z})$ and $E_{4}^{*, *}\left(\mathbf{e}^{\prime}\right)=E_{4}^{*, *}\left(\mathbf{e}^{\prime}, \mathbf{Z}\right)$ for short, and where

$$
B_{j}=\frac{k_{j}^{\prime}}{k_{j}}\left(1+\left(t^{j}\right)+\cdots+\left(t^{j}\right)^{p-1}\right) .
$$

In the diagram, the notations $B_{j}$ and $h_{j-1}^{\prime} / h_{j-1}$ are intended to indicate that the generators

$$
\frac{r}{k_{j}} c_{x}^{i} \omega_{x} c_{y}^{j} \in E_{3}^{2 i+1,2 j}(\mathbf{e}, \mathbf{Z}) \cong \mathbf{Z} / q_{j}
$$


and

$$
\frac{r}{h_{j-1}} c_{x}^{i+2} c_{y}^{j-1} \in E_{3}^{2 i+4,2 j-2}(\mathbf{e}, \mathbf{Z}) \cong \mathbf{Z} / q_{j-1}
$$

are mapped to

$$
B_{j} \cdot \frac{r}{k_{j}^{\prime}} c_{x^{\prime}}^{i} \omega_{x^{\prime}} c_{y}^{j} \in E_{3}^{2 i+1,2 j}\left(\mathbf{e}^{\prime}, \mathbf{Z}\right) \cong \mathbf{Z} / q_{j}^{\prime}
$$

and

$$
\frac{h_{j-1}^{\prime}}{h_{j-1}} \cdot \frac{r}{h_{j-1}^{\prime}} c_{x^{\prime}}^{i+2} c_{y}^{j-1} \in E_{3}^{2 i+4,2 j-2}\left(\mathbf{e}^{\prime}, \mathbf{Z}\right) \cong \mathbf{Z} / q_{j-1}^{\prime},
$$

respectively.

Corollary 7.4. On $E_{4}$ the restriction map $E_{4}(\mathbf{e}, \mathbf{Z}) \rightarrow E_{4}\left(\mathbf{e}^{\prime}, \mathbf{Z}\right)$ is given by

$$
\operatorname{res}\left(c_{x}\right)=c_{x^{\prime}}, \quad \operatorname{res}\left(c_{2 j}\right)=\frac{h_{j}^{\prime}}{h_{j}} c_{2 j}^{\prime},
$$

and, if $r_{p} \geq s_{p}$ and $\nu \not \equiv 0 \bmod \left(q_{1}\right)_{p}$,

$$
\operatorname{res}\left(x_{2 j+1}\right)=U\left(1+\left(t^{j}\right)+\cdots+\left(t^{j}\right)^{p-1}\right) x_{2 j+1}^{\prime},
$$

where $U$ is a unit modulo $p$.

The exact value of $U$ will be given in the proof.

Proof. To compute res $\left(x_{2 j+1}\right)$ under the mentioned circumstances, we recall that

$$
x_{2 j+1}=\frac{q_{1}}{\left(\nu, q_{1}\right)} \frac{r}{k_{j}} \omega_{x} c_{y}^{j} .
$$

In the typical case, we have

$$
\begin{aligned}
\operatorname{res}\left(x_{2 j+1}\right) & =\left(1+\left(t^{j}\right)+\cdots+\left(t^{j}\right)^{p-1}\right) \frac{q_{1}}{\left(\nu, q_{1}\right)} \frac{r}{k_{j}} \omega_{x^{\prime}} c_{y}^{j} \\
& =\left(1+\left(t^{j}\right)+\cdots+\left(t^{j}\right)^{p-1}\right) p \frac{q_{1} k_{j}^{\prime}}{q_{1}^{\prime} k_{j}} \frac{q_{1}^{\prime}}{\left(\nu^{\prime}, q_{1}^{\prime}\right)} \frac{r}{k_{j}^{\prime}} \omega_{x^{\prime}} c_{y}^{j} \\
& =U\left(1+\left(t^{j}\right)+\cdots+\left(t^{j}\right)^{p-1}\right) x_{2 j+1}^{\prime},
\end{aligned}
$$

where $U=p \frac{q_{1} k_{j}^{\prime}}{q_{1}^{\prime} k_{j}}$. In view of (3.3), $U$ is a unit at $p$. In the exceptional case, the extension $\mathbf{e}^{\prime}$ splits at $2, x_{2 j+1}^{\prime}=\frac{r}{k_{j}^{\prime}} \omega_{x^{\prime}} c_{y}^{j}$, and hence

$$
\begin{aligned}
\operatorname{res}\left(x_{2 j+1}\right) & =\left(1+t^{j}\right) \frac{q_{1}}{\left(\nu, q_{1}\right)} \frac{r}{k_{j}} \omega_{x^{\prime}} c_{y}^{j} \\
& =\left(1+t^{j}\right) \frac{q_{1} k_{j}^{\prime}}{\left(\nu, q_{1}\right) k_{j}} \frac{r}{k_{j}^{\prime}} \omega_{x^{\prime}} c_{y}^{j}=U\left(1+t^{j}\right) x_{2 j+1}^{\prime},
\end{aligned}
$$

where $U=\frac{q_{1} k_{j}^{\prime}}{\left(\nu, q_{1}\right) k_{j}}$. In view of (3.4), $\nu$ is odd, $\left(q_{1}\right)_{2}=2$, and $U$ is odd. 
The other statements are straightforward or obvious.

As before we denote by $d$ the order of $t$ modulo $r$. We remind the reader that, in view of (3.2), in the typical case $d_{p}=\left(\frac{r}{h_{1}}\right)_{p}$.

Lemma 7.5. In the typical case, if $r_{p} \geq s_{p}$ and $\nu \not \equiv 0 \bmod \left(q_{1}\right)_{p}$, the induced morphism $E_{4}^{k, l}(\mathbf{e}, \mathbf{Z}) \longrightarrow E_{4}^{k, l}\left(\mathbf{e}^{\prime}, \mathbf{Z}\right)$ enjoys the following properties:

(1) It is injective unless

$$
(k, l)= \begin{cases}(2,2 j), & \text { or } \\ \left(1,2 \mu d_{p}\right), & \text { or possibly } \\ \left(2 i+1,2 \mu d_{p}\right), & \text { for } i \geq 1,\end{cases}
$$

where $j \geq 0$ and $\mu \geq 1$.

(2) It is surjective at the prime $p$ only if $(k, l)=\left(2 i, 2 j d_{p}\right)$. Whenever it is not surjective at the prime $p$, its cokernel at $p$ is a copy of $\mathbf{Z} / p$.

(3) The kernel of the induced morphism

$$
E_{4}^{2,2 j}(\mathbf{e}, \mathbf{Z})=\mathbf{Z} / q_{j}\left\langle c_{x} c_{2 j}\right\rangle \longrightarrow E_{4}^{2,2 j}\left(\mathbf{e}^{\prime}, \mathbf{Z}\right)=\mathbf{Z} / q_{j}^{\prime}\left\langle c_{x}^{\prime} c_{2 j}^{\prime}\right\rangle
$$

is a copy of $\mathbf{Z} / p$, generated by (the class of) $\frac{q_{j}}{p} c_{x} c_{2 j}$.

(4) For $l=\mu d_{p}, \mu \geq 1$, the kernel of the induced morphism

$$
E_{4}^{1,2 l}(\mathbf{e}, \mathbf{Z}) \longrightarrow E_{4}^{1,2 l}\left(\mathbf{e}^{\prime}, \mathbf{Z}\right)
$$

is a copy of $\mathbf{Z} / p$, generated by (the class of) $d_{p}\left(\nu, q_{1}\right) x_{2 l+1} / p$.

Proof. Away from $p$ there is nothing to prove. Furthermore, in view of (0.4), (6.7), and (6.9), statement (3) is immediate. Since by hypothesis $r_{p} \geq s_{p}$ and $\nu \not \equiv 0 \bmod \left(q_{1}\right)_{p}$, Theorem 0.4 together with (6.7) implies that the differential $d_{3}$ is nontrivial at $p$. Now (3.3) implies at once that the number $h_{j-1}^{\prime} / h_{j-1}$ equals 1 or $p$ according as $(j-1)$ is or is not divisible by $d_{p}$; hence the morphism in (7.3) labelled by this number has as kernel a copy of $\mathbf{Z} / p$ but since the differential $d_{3}$ is nontrivial at $p$ this copy of $\mathbf{Z} / p$ is killed and does not survive to $E_{4}$, whence the induced morphism

$$
E_{4}^{2 i+4,2 j-2}(\mathbf{e}, \mathbf{Z}) \longrightarrow E_{4}^{2 i+4,2 j-2}\left(\mathbf{e}^{\prime}, \mathbf{Z}\right)
$$

is injective. This settles part of statement (1). Likewise (3.3) implies at once that the number $B_{j}$ in (7.3) is prime to $p$; hence the morphism in (7.3) labelled by this number is an isomorphism at the prime $p$ if $j$ is not divisible by $d_{p}=\left(\frac{r}{h_{1}}\right)_{p}$ and it is surjective at the prime $p$ with kernel a copy of $\mathbf{Z} / p$ if $j$ is divisible by $d_{p}$. Consequently, at the prime $p$, the induced morphism

$$
E_{4}^{2 i+1,2 j}(\mathbf{e}, \mathbf{Z}) \longrightarrow E_{4}^{2 i+1,2 j}\left(\mathbf{e}^{\prime}, \mathbf{Z}\right)
$$

has kernel a copy of $\mathbf{Z} / p$ or is injective according as $j$ is or is not divisible by $d_{p}$. This settles the corresponding statements in (1) and (4). The proof of the remaining statements is straightforward and left to the reader. 
Recall that, in view of $(3.2), d_{2}=\frac{r}{(t+1, r / 2)}$ in the exceptional case. Recall also that, in view of (6.10), in the exceptional case, $E_{4}^{k, l}(\mathbf{e}, \mathbf{Z})$ is nonzero at the prime 2 only if $l$ is even and $k=0,2$ or if $l$ is divisible by 4 . Moreover, in this case, in view of (3.4), if $r_{2} \geq s_{2}$, the extension $\mathbf{e}^{\prime}$ splits at the prime 2 .

Lemma 7.6. In the exceptional case, if $r_{2} \geq s_{2}$ and $\nu \not \equiv 0 \bmod \left(q_{1}\right)_{2}$, the induced morphism

$$
E_{4}^{k, l}(\mathbf{e}, \mathbf{Z}) \longrightarrow E_{4}^{k, l}\left(\mathbf{e}^{\prime}, \mathbf{Z}\right)
$$

enjoys the following properties:

(1) If $l \equiv 0 \bmod 4$ it is injective unless $k=2$ or

$$
(k, l)=(2 i+1,2 j), \quad \text { with } i \geq 0, j \text { even, and } j(t+1) \text { divisible by } r_{2} \text {, }
$$

while if $l \equiv 2 \bmod 4$ and $k$ is odd, $E_{4}^{k, l}(\mathbf{e}, \mathbf{Z})$ is zero anyway, whence the induced morphism is injective.

(2) It is surjective at the prime 2 only if

$$
(k, l)=(2 i, 2 j) \text {, with } i \geq 0, j \text { even, and } j(t+1) \text { divisible by } r_{2} .
$$

Whenever it is not surjective at the prime 2 , its cokernel is a copy of $\mathbf{Z} / 2$.

(3) For $j$ even, the kernel of the induced morphism

$$
E_{4}^{2,2 j}(\mathbf{e}, \mathbf{Z})=\mathbf{Z} / q_{j}\left\langle c_{x} c_{2 j}\right\rangle \longrightarrow E_{4}^{2,2 j}\left(\mathbf{e}^{\prime}, \mathbf{Z}\right)=\mathbf{Z} / q_{j}^{\prime}\left\langle c_{x^{\prime}} c_{2 j}^{\prime}\right\rangle
$$

is a copy of $\mathbf{Z} / 2$, generated by (the class of) $\frac{k_{j}}{2} c_{x} c_{y}^{j}=\frac{q_{j}}{2} c_{x} c_{2 j}$.

(4) For $l=2 j$, with $j$ even and $j(t+1)$ divisible by $r_{2}$, the kernel of the induced morphism

$$
E_{4}^{1,2 j}(\mathbf{e}, \mathbf{Z}) \longrightarrow E_{4}^{1,2 j}\left(\mathbf{e}^{\prime}, \mathbf{Z}\right)
$$

is a copy of $\mathbf{Z} / 2$, generated by (the class of) $q_{j}^{\prime} x_{2 j+1} / 2$.

Proof. Away from 2 there is nothing to prove. Furthermore, in view of (0.4), (6.7), and (6.9), statement (3) is again immediate. Theorem 0.4 together with (6.7) implies that the differential $d_{3}$ is nontrivial at 2 , and in view of what was said above, the groups $E_{4}^{k, l}(\mathbf{e}, \mathbf{Z})$ are nontrivial only if $k=0,2$ and $l$ is even or if $l$ is divisible by 4. Now for $j$ odd, (3.4) implies at once that the number $h_{j-1}^{\prime} / h_{j-1}$ equals 1 or 2 according as $(j-1)(t+1)$ is or is not divisible by $r_{2}$; hence the morphism in (7.3) labelled by this number has as kernel a copy of $\mathbf{Z} / 2$ but since the differential $d_{3}$ is nontrivial at the prime 2 this copy of $Z / 2$ is killed and does not survive to $E_{4}$, whence the induced morphism

$$
E_{4}^{2 i+4,2 j-2}(\mathbf{e}, \mathbf{Z}) \longrightarrow E_{4}^{2 i+4,2 j-2}\left(\mathbf{e}^{\prime}, \mathbf{Z}\right)
$$

is injective. This settles part of statement (1). Likewise (3.4) implies at once that for $j$ even the number $B_{j}$ in (7.3) is odd; hence the morphism labelled by this number is an isomorphism at the prime 2 if $j(t+1)$ is not divisible by $r_{2}$ and it is surjective at the prime 2 with kernel a copy of $\mathbf{Z} / 2$ if $j(t+1)$ 
is divisible by $r_{2}$. This implies readily that, at the prime 2 , for $j$ even, the induced morphism

$$
E_{4}^{2 i+1,2 j}(\mathbf{e}, \mathbf{Z}) \longrightarrow E_{4}^{2 i+1,2 j}\left(\mathbf{e}^{\prime}, \mathbf{Z}\right)
$$

has kernel a copy of $\mathbf{Z} / 2$ or is injective according as $j(t+1)$ is or is not divisible by $r_{2}$. This settles the corresponding statements in (1) and (4). The proof of the remaining statements is again straightforward and left to the reader.

Corollary 7.7. Let $p$ be a prime dividing $r$ and $s$, assume that $r_{p} \geq s_{p}$, and suppose that the extension e does not split at $p$, i.e., that $\nu_{p} \not \equiv 0 \bmod \left(q_{1}\right)_{p}$. Then the restriction map $E_{\infty}(\mathbf{e}, \mathbf{Z}) \rightarrow E_{\infty}\left(\mathbf{e}^{\prime}, \mathbf{Z}\right)$ sends $c_{x}$ to $c_{x^{\prime}}$ and, on the remaining generators, it is given by the following rules:

(1) In the typical case,

$$
\begin{gathered}
\operatorname{res}\left(c_{2 j}\right)=\frac{h_{j}^{\prime}}{h_{j}} c_{2 j}^{\prime}= \begin{cases}U p c_{2 j}^{\prime}, & \text { if } j \text { is not divisible by } d_{p}, \\
V c_{2 j}^{\prime}, & \text { if } j \text { is divisible by } d_{p},\end{cases} \\
\operatorname{res}\left(x_{2 j+1}\right)=p W x_{2 j+1}^{\prime},
\end{gathered}
$$

where $U, V, W$ are units modulo $p$.

(2) In the exceptional case,

$$
\begin{aligned}
\operatorname{res}\left(c_{2 j}\right)=\frac{h_{j}^{\prime}}{h_{j}} c_{2 j}^{\prime}= \begin{cases}\left(t+1, \frac{r}{2}\right) c_{2 j}^{\prime}, & \text { if } j \text { is odd, } \\
2 U c_{2 j}^{\prime}, & \text { if } j \text { is even and } j(t+1) \neq 0 \bmod r_{2}, \\
V c_{2 j}^{\prime}, & \text { if } j \text { is even and } j(t+1) \equiv 0 \bmod r_{2}, \\
& \operatorname{res}\left(x_{2 j+1}\right)=2 W x_{2 j+1}^{\prime}, \quad j \text { even, }\end{cases}
\end{aligned}
$$

where $U, V, W$ are odd numbers.

Proof. By (6.11) the spectral sequence has $E_{4}=E_{\infty}$ at the prime $p$. Hence the assertions follow from (7.4), (7.5), (7.6), and the number theory in $\S 3$.

\section{Proof of Theorem 0.3: The additive EXtension Problem}

As before, let $p$ be a prime dividing $q_{1}=\left|\mathrm{H}^{2}(K, N)\right|$, and suppose that $\nu \not \equiv$ $0 \bmod \left(q_{1}\right)_{p}$ and that $r_{p} \geq s_{p}$, so that, in view of (6.12), the prime $p$ is good for the presentation ( 0.1 ) (cf. what was said in the Introduction). Moreover, let $G^{\prime}$ be the subgroup of $G$ generated by $y$ and $x^{\prime}=x^{p}$, with presentation

$$
G^{\prime}=G\left(r, s^{\prime}, t^{\prime}, f\right)=\left\langle x^{\prime}, y ; y^{r}=1, x^{s^{\prime}}=y^{f}, x^{\prime} y x^{\prime-1}=y^{t^{\prime}}\right\rangle,
$$

where $s^{\prime}=s / p$ and $t^{\prime}=t^{p}$. It fits into the group extension

$$
1 \rightarrow G^{\prime} \rightarrow G \stackrel{\mathrm{pr}}{\longrightarrow} \mathbf{Z} / p \rightarrow 1
$$


and the injection maps yield a commutative diagram

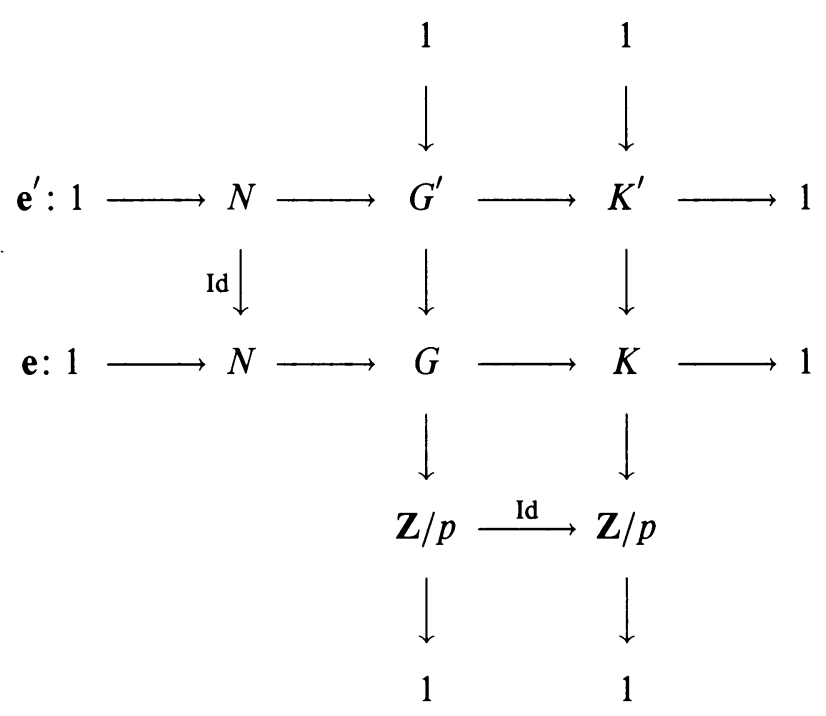

We now assume by induction that Theorem 0.3 has been proved for the extension $\mathbf{e}^{\prime}$. We note that, in view of (2.8), (2.22), (2.24), and (2.27), the induction starts at the extension (4.5) or (4.12) as appropriate. We verify the statement of Theorem 0.3 for e by comparing the cohomologies of $G$ and $G^{\prime}$, viewed as filtered objects: Recall that the cohomologies of $G$ and $G^{\prime}$ inherit filtrations from the extension $\mathbf{e}$ and $\mathbf{e}^{\prime}$, respectively. Since $E_{2}^{*, \text { odd }}(\mathbf{e}, \mathbf{Z})$ and $E_{2}^{\text {odd }, 0}(\mathbf{e}, \mathbf{Z})$ are zero, the filtration of $\mathrm{H}^{*}(G, \mathbf{Z})$ looks like

$$
\begin{aligned}
\mathrm{H}^{2 j}(G, \mathbf{Z}) & =\mathrm{H}^{0,2 j}(\mathbf{e}) \supseteq \mathrm{H}^{2,2 j-2}(\mathbf{e}) \supseteq \cdots \supseteq \mathrm{H}^{2 j, 0}(\mathbf{e}), \\
\mathrm{H}^{2 j+1}(G, \mathbf{Z}) & =\mathrm{H}^{1,2 j}(\mathbf{e}) \supseteq \mathrm{H}^{3,2 j-2}(\mathbf{e}) \supseteq \cdots \supseteq \mathrm{H}^{2 j-1,2}(\mathbf{e})
\end{aligned}
$$

and the same remark applies to $G^{\prime}$ and $\mathbf{e}^{\prime}$.

Lemma 8.3.t. In the typical case, for $j$ not divisible by $d_{p}$, the restriction map

$$
\mathrm{H}^{2 j+1}(G) \longrightarrow \mathrm{H}^{2 j+1}\left(G^{\prime}\right)
$$

is injective while, for $j=\mu d_{p}$, the morphism

$$
\mathrm{H}^{3,2 j-2}(\mathbf{e}) \longrightarrow \mathrm{H}^{3,2 j-2}\left(\mathbf{e}^{\prime}\right)
$$

is injective and the restriction map

$$
\mathrm{H}^{2 j+1}(G)=\mathrm{H}^{1,2 j}(\mathbf{e}) \longrightarrow \mathrm{H}^{1,2 j}\left(\mathbf{e}^{\prime}\right)=\mathrm{H}^{2 j+1}\left(G^{\prime}\right)
$$

has as kernel a copy of $\mathbf{Z} / p$, generated by

$$
d_{p}\left(\nu, q_{1}\right) \xi_{2 j+1}+\text { possibly terms of higher filtration. }
$$


Proof. In view of (7.5), a straightforward induction shows that, for $i \geq 0$ and $j \geq 1$, the diagram

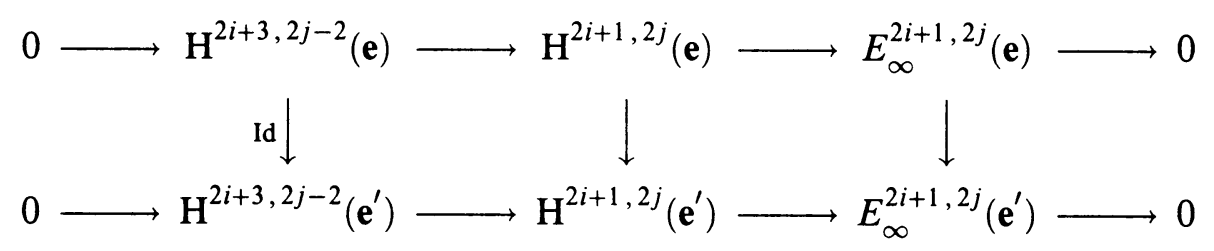

has injective columns unless possibly $j$ is divisible by $2 d_{p}$. For $i=0$ and $j=\mu d_{p}$, we have a diagram

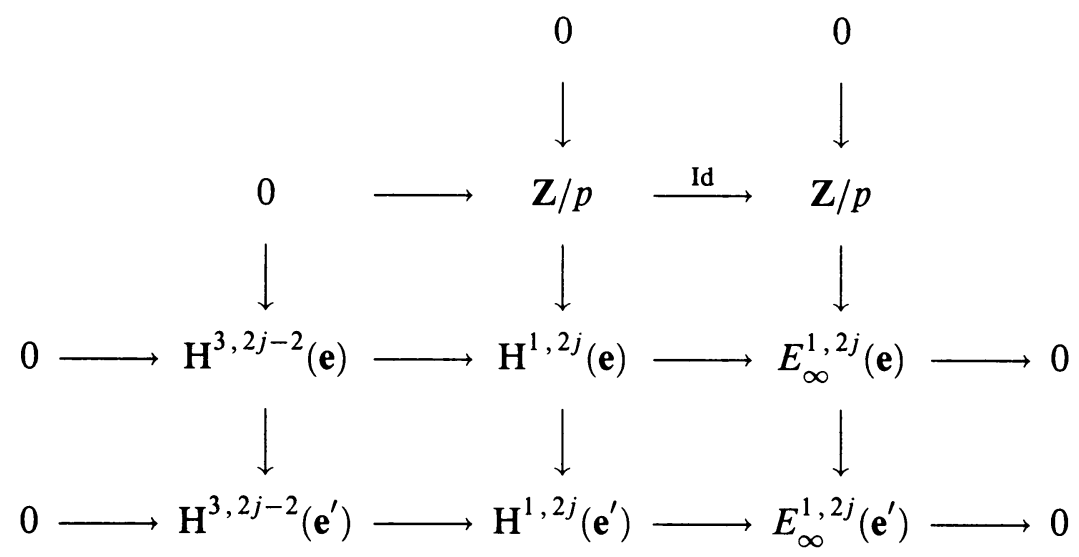

with exact rows and columns. By (7.5)(4) the kernel of the induced morphism

$$
E_{\infty}^{1,2 \mu d_{p}}(\mathbf{e}) \longrightarrow E_{\infty}^{1,2 \mu d_{p}}\left(\mathbf{e}^{\prime}\right)
$$

is a copy of $\mathbf{Z} / p$, generated by $d_{p}\left(\nu, q_{1}\right) x_{2 \mu d_{p}+1} / p$. In view of (4.22), (6.9), and (6.11), we conclude that the kernel of $\mathbf{H}^{1,2 \mu d_{p}}(\mathbf{e}) \rightarrow \mathbf{H}^{1,2 \mu d_{p}}\left(\mathbf{e}^{\prime}\right)$ is generated by

$$
\frac{d_{p}}{p}\left(\nu, q_{1}\right) \xi_{2 \mu d_{p}+1}+\text { possibly terms of higher filtration. }
$$

Lemma 8.3.e. In the exceptional case, for $j$ even and $j(t+1)$ not divisible by $r_{2}$, the restriction map $\mathrm{H}^{2 j+1}(G) \rightarrow \mathrm{H}^{2 j+1}\left(G^{\prime}\right)$ is injective, while for $j$ even and $j(t+1)$ divisible by $r_{2}$, the morphism $\mathrm{H}^{3,2 j-2}(\mathbf{e}) \rightarrow \mathrm{H}^{3,2 j-2}\left(\mathbf{e}^{\prime}\right)$ is injective and the restriction map

$$
\mathrm{H}^{2 j+1}(G)=\mathrm{H}^{1,2 j}(\mathbf{e}) \longrightarrow \mathrm{H}^{1,2 j}\left(\mathbf{e}^{\prime}\right)=\mathrm{H}^{2 j+1}\left(G^{\prime}\right)
$$

has as kernel a copy of $\mathbf{Z} / 2$, generated by

$$
\frac{q_{j}^{\prime}}{2} \xi_{2 j+1}+\text { possibly terms of higher filtration. }
$$

Proof. This is virtually identical to that of the previous lemma, with (7.6) instead of (7.5). We leave the details to the reader. 
We can now verify the relations $(0.3 .4)$ for $G$ and $\mathbf{e}$ : In view of the inductive hypothesis, we have the defining relations

$$
\frac{q_{j}^{\prime}}{q_{1}^{\prime}}\left(\nu^{\prime}, q_{1}^{\prime}\right) \xi_{2 j+1}^{\prime}=0
$$

for $G^{\prime}$ and $\mathbf{e}^{\prime}$. In the exceptional case, since by hypothesis $r_{2} \geq s_{2}$, in view of (3.4), the extension $\mathbf{e}^{\prime}$ splits at 2 , and these relations boil down to

$$
q_{j}^{\prime} \xi_{2 j+1}^{\prime}=0
$$

Lemma 8.5. In the typical case,

$$
\left(\frac{q_{j}}{q_{1}}\left(\nu, q_{1}\right)\right)_{p}= \begin{cases}\left(\frac{q_{j}^{\prime}}{q_{1}^{\prime}}\left(\nu^{\prime}, q_{1}^{\prime}\right)\right)_{p} / p, & \text { whenever } j \text { is not divisible by } d_{p}, \\ \left(\frac{q_{j}^{\prime}}{q_{1}^{\prime}}\left(\nu^{\prime}, q_{1}^{\prime}\right)\right)_{p}, & \text { if } j \text { is divisible by } d_{p},\end{cases}
$$

while in the exceptional case, for $j$ even,

$$
\left(\frac{q_{j}}{q_{1}}\left(\nu, q_{1}\right)\right)_{2}= \begin{cases}\left(q_{j}^{\prime}\right)_{2} / 2, & \text { whenever } j(t+1) \text { is not divisible by } r_{2}, \\ \left(q_{j}^{\prime}\right)_{2}, & \text { if } j(t+1) \text { is divisible by } r_{2} .\end{cases}
$$

Proof. By virtue of (3.3), in the typical case, $\left(\nu^{\prime}, q_{1}^{\prime}\right)_{p}=p\left(\nu, q_{1}\right)_{p}$, and

$$
\left(\frac{q_{j}}{q_{1}}\right)_{p}=p\left(\frac{q_{j}^{\prime}}{q_{1}^{\prime}}\right)_{p} \text { or }\left(\frac{q_{j}}{q_{1}}\right)_{p}=\left(\frac{q_{j}^{\prime}}{q_{1}^{\prime}}\right)_{p}
$$

according as $j$ is or is not divisible by $d_{p}$. This implies at once (8.5.1). In the exceptional case, by (3.4), $\left(q_{1}\right)_{2}=2, \nu$ is odd, and, for $j$ even, $\left(q_{j}\right)_{2}=2\left(q_{j}^{\prime}\right)_{2}$ or $\left(q_{j}\right)_{2}=\left(q_{j}^{\prime}\right)_{2}$ according as $j(t+1)$ is or is not divisible by $r_{2}$. This implies at once (8.5.2).

To verify the relations $(0.3 .4)$ for $1 \leq j \leq d_{p}$, we recall from (4.21) that, by construction,

$$
\xi_{2 j+1}=\tau_{G^{\prime} \uparrow G}\left(\xi_{2 j+1}^{\prime}\right) \in \mathbf{H}^{2 j+1}(G, \mathbf{Z}) .
$$

By induction, at the prime $p$, the class $\xi_{2 j+1}^{\prime}$ has exact order $\frac{q_{j}^{\prime}}{q_{1}^{\prime}}\left(\nu^{\prime}, q_{1}^{\prime}\right)$. Hence at the prime $p$ the class $\xi_{2 j+1}$ has at most this order. Moreover (cf. (4.22), (6.9), and (6.11)), the class $\xi_{2 j+1}$ passes to the class $x_{2 j+1}$ in the associated graded object of order

$$
\frac{q_{j}^{\prime}}{q_{1}^{\prime}}\left(\nu{ }^{\prime}, q_{1}^{\prime}\right) / p=\frac{q_{j}}{q_{1}}\left(\nu, q_{1}\right)
$$

at the prime $p$. Hence at $p$ the class $\xi_{2 j+1}$ has at least order $\frac{q_{j}}{q_{1}}\left(\nu, q_{1}\right)$. However, it is well known that the composite

$$
\mathrm{H}^{*}(G, \mathbf{Z}) \stackrel{\text { res }}{\longrightarrow} \mathrm{H}^{*}\left(G^{\prime}, \mathbf{Z}\right) \stackrel{{ }^{\tau_{G^{\prime} \uparrow G}} \longrightarrow}{\longrightarrow} \mathrm{H}^{*}(G, \mathbf{Z})
$$


is just multiplication by the index $p=\left[G: G^{\prime}\right]$, whence

$$
\tau_{G^{\prime} \uparrow G}\left(\operatorname{res}\left(\xi_{2 j+1}\right)\right)=p \xi_{2 j+1} \in \mathrm{H}^{2 j+1}(G, \mathbf{Z}) .
$$

In the typical case, by (8.3.t) above, for $1 \leq j<d_{p}$, the restriction map

$$
\mathrm{H}^{2 j+1}(G, \mathbf{Z}) \longrightarrow \mathrm{H}^{2 j+1}\left(G^{\prime}, \mathbf{Z}\right)
$$

is injective. If at $p$ the class $\xi_{2 j+1}$ had order $p \frac{q_{j}}{q_{1}}\left(\nu, q_{1}\right)$, the restriction of the transfer $\tau_{G^{\prime} \uparrow G}$ to the cyclic subgroup of $\mathrm{H}^{2 j+1}\left(G^{\prime}, \mathbf{Z}\right)$ generated by $\xi_{2 j+1}^{\prime}$ would be an isomorphism and hence the restriction of the composite $\tau_{G^{\prime} \uparrow G}$ ores to this cyclic subgroup would be injective; however this is impossible since $\tau_{G^{\prime} \uparrow G} \circ$ res is multiplication by $p$. Hence at the prime $p$ the class $\xi_{2 j+1}$ has exact order

$$
\frac{q_{j}^{\prime}}{q_{1}^{\prime}}\left(\nu^{\prime}, q_{1}^{\prime}\right) / p=\frac{q_{j}}{q_{1}}\left(\nu, q_{1}\right)
$$

and the relation

$$
\frac{q_{j}}{q_{1}}\left(\nu, q_{1}\right) \xi_{2 j+1}=0
$$

is a defining relation for $1 \leq j<d_{p}$. For $j=d_{p}$, we observe that the class $\xi_{2 j+1}$ passes to the class $x_{2 j+1} \in E_{\infty}$ and in view of (6.9) and (6.11) the latter has exact order

$$
\frac{q_{j}^{\prime}}{q_{1}^{\prime}}\left(\nu^{\prime}, q_{1}^{\prime}\right)=\frac{q_{j}}{q_{1}}\left(\nu, q_{1}\right), \quad j=d_{p} .
$$

This proves the relations (0.3.4) for $1 \leq j \leq d_{p}$. However, in view of the multiplicative structure and the construction of the classes $\xi_{2 j+1}$ for $j>d_{p}$ in (4.20), it is clear that these relations hold for every $j \geq 1$. One only has to observe that the classes $\zeta_{2 j}$ have order at least $h_{j}$ which follows at once from (6.9) and (6.11) and that for each $j$ the number $h_{j}$ is divisible by $q_{j}=\frac{h_{j} k_{j}}{r}$ and hence by $\frac{q_{j}}{q_{1}}\left(\nu, q_{1}\right)$. Hence the relations $(0.3 .4)$ are defining relations in the typical case.

In the exceptional case with $j$ even we proceed as in the typical case with (8.3.e) instead of (8.3.t). Furthermore, we know from (6.10) that $E_{\infty}^{1,2 j}$ is zero for $j$ odd and hence we have completely determined the structure of $\mathrm{H}^{\text {odd }}(G)$ in the exceptional case also.

Next we study the structure of $\mathrm{H}^{\text {even }}(G)$.

Lemma 8.6. For $j \geq 1$, the induced morphism $\mathrm{H}^{4,2 j-4}(\mathbf{e}) \rightarrow \mathrm{H}^{4,2 j-4}\left(\mathbf{e}^{\prime}\right)$ is injective (with both groups interpreted to be zero for $j=1$ ), while the induced morphism

$$
\mathrm{H}^{2,2 j-2}(\mathbf{e}) \longrightarrow \mathrm{H}^{2,2 j-2}\left(\mathbf{e}^{\prime}\right)
$$

has as kernel a copy of $\mathbf{Z} / p$, generated by

$$
\frac{q_{j}}{p} c_{x} \zeta_{2 j}+\text { possibly terms of higher filtration, }
$$


and this kernel coincides with that of the restriction map

$$
\mathrm{H}^{2 j}(G)=\mathrm{H}^{0,2 j}(\mathbf{e}, \mathbf{Z}) \longrightarrow \mathrm{H}^{0,2 j}\left(\mathbf{e}^{\prime}, \mathbf{Z}\right)=\mathrm{H}^{2 j}\left(G^{\prime}\right) .
$$

We note that, in the exceptional case with $j$ odd, $\left(q_{j}\right)_{2}=2$, and the lemma then says in particular that the kernel of the restriction map is generated by

$$
c_{x} \zeta_{2 j}+\text { possibly terms of higher filtration. }
$$

Proof. In view of (7.5), a straightforward induction shows that, for $i \geq 2$ and $j \geq 1$, the diagram

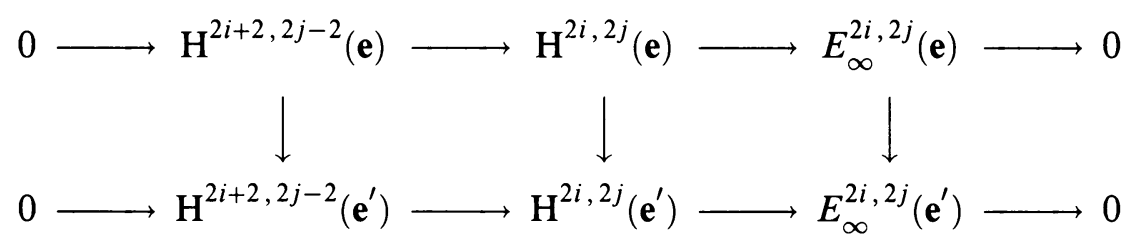

has injective columns unless $i=0$ or $i=1$, while, for $i=1$, the corresponding diagram looks like

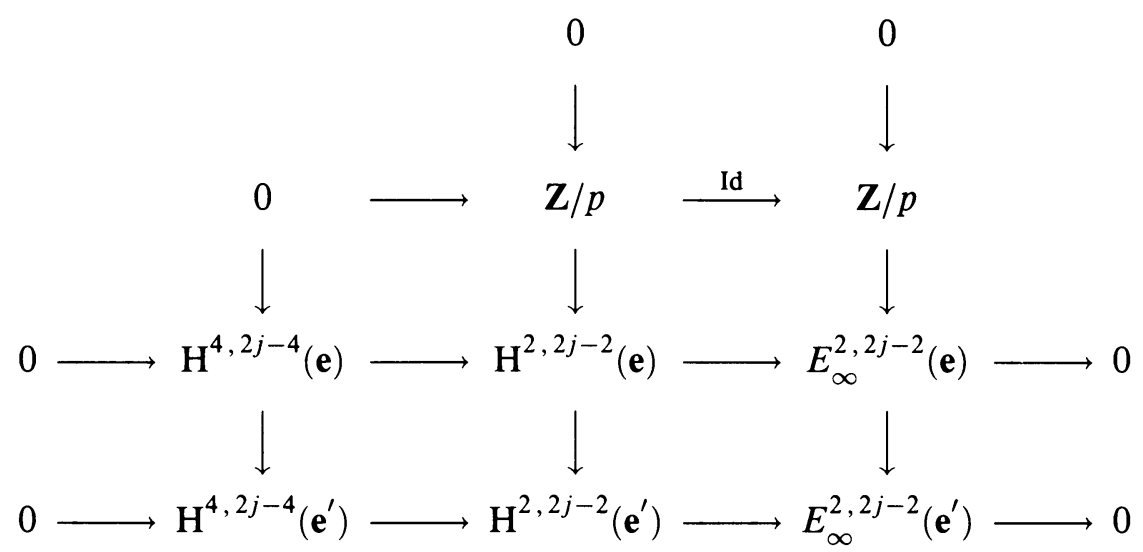

with exact rows and columns; we note that, when $\mathrm{H}^{4,-2}$ is interpreted to be zero, this makes sense for $j=1$, too. In the typical case, in view of (7.5)(3), and in the exceptional case with $j$ odd, in view of (7.6)(3), the kernel of $E_{\infty}^{2,2 j-2}(\mathbf{e}) \rightarrow E_{\infty}^{2,2 j-2}\left(\mathbf{e}^{\prime}\right)$ is generated by $\frac{q_{j}}{p} c_{x} c_{2 j}$, whence, in view of (4.22), (6.9), and (6.11), the kernel of $\mathrm{H}^{2,2 j-2}(\mathbf{e}) \rightarrow \mathrm{H}^{2,2 j-2}\left(\mathbf{e}^{\prime}\right)$ is generated by

$$
\frac{q_{j}}{p} c_{x} \zeta_{2 j}+\text { possibly terms of higher filtration. }
$$

In the exceptional case with $j$ even, in view of (6.10), at the prime 2 the group $E_{\infty}^{2,2 j-2}(\mathbf{e})$ is cyclic of order 2 and the induced map $E_{\infty}^{2,2 j-2}(\mathbf{e}) \rightarrow E_{\infty}^{2,2 j-2}\left(\mathbf{e}^{\prime}\right)$ 
is trivial. Likewise, for $i=0$, we have such a diagram

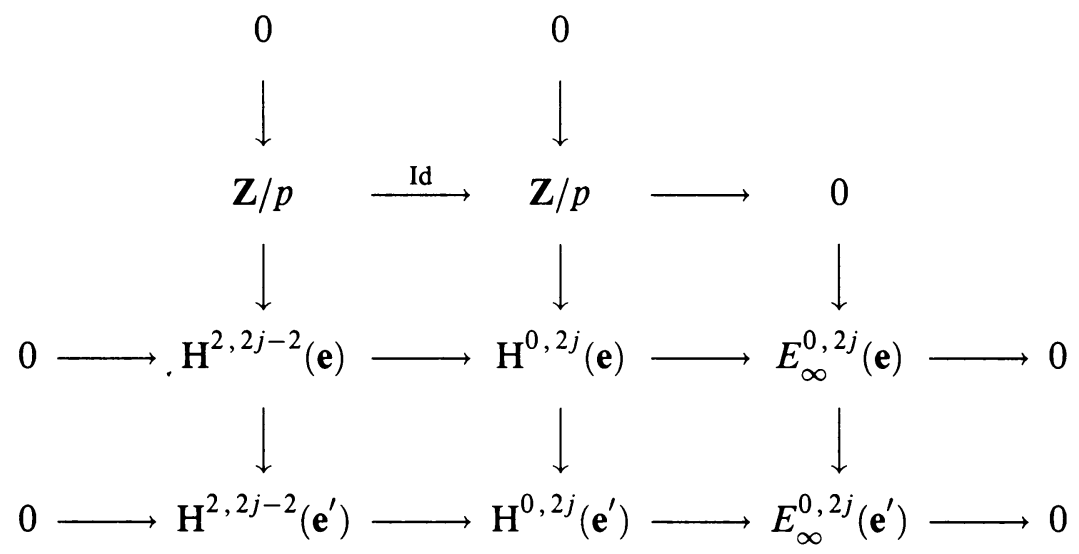

with exact rows and columns.

We note that, for $i=0$, in the typical case, at $p$ the induced morphism $E_{\infty}^{0,2 j}(\mathbf{e}) \rightarrow E_{\infty}^{0,2 j}\left(\mathbf{e}^{\prime}\right)$ is in fact an isomorphism for $j \equiv 0 \bmod d_{p}$ in view of (7.5) and in the exceptional case at 2 if $j(t+1) \equiv 0 \bmod r_{2}$ in view of (7.6).

The relations (0.3.3) in the typical case and for $j$ even in the exceptional case follow from the inductive assumption and Lemmas 8.5 and 8.6 in much the same way as the relations (0.3.4) follow from the inductive assumption, combined with Lemma 8.5 and Lemmas 8.3.t or 8.3.e, according as we are in the typical or exceptional case. We leave the details to the reader.

To verify the relations $(0.3 .1)$ and (0.3.2) in the typical case we need some more preparations. We proceed as follows.

Lemma 8.7. At each prime $p$ so that $r_{p} \geq s_{p}$ the following hold.

(1) For $j \geq 1$,

$$
\begin{aligned}
\left(1+\left(t^{j}\right)+\cdots+\left(t^{j}\right)^{s-1}\right)_{p} & =\left(1+\left(t^{j}\right)+\cdots+\left(t^{j}\right)^{s-1}, r\right)_{p}=\left(k_{j}\right)_{p} \\
\left(h_{j} \frac{s}{f_{p}}\right)_{p} & =\left(h_{j} \frac{q_{1}}{\left(\nu, q_{1}\right)}\right)_{p}
\end{aligned}
$$

unless we are in the exceptional case and $j$ is odd.

(2) In the exceptional case, if $j$ is odd,

$$
\left(1+\left(t^{j}\right)+\cdots+\left(t^{j}\right)^{s-1}\right)_{2}=\left(\frac{s}{2}(t+1)\right)_{2}
$$

while

$$
\left(k_{j}\right)_{2}=\left(\frac{s}{2}(t+1), r_{2}\right)_{2},
$$

and the latter equals $r_{2}$ if $\nu \not \equiv 0 \bmod \left(q_{1}\right)_{2}$.

Proof. The relation (8.7.1) is a consequence of $(3.1)(1)$. Likewise, to see that (8.7.2) holds, we recall that $f=\nu \frac{r}{h_{1}}$ and, by virtue of $(3.2)$, that $\left(k_{1}\right)_{p}=s_{p}$ in 
the typical case since by hypothesis $r_{p} \geq s_{p}$. Since $q_{1}=\frac{k_{1} h_{1}}{r}$, we conclude

$$
\left(h_{j} \frac{s}{f_{p}}\right)_{p}=\left(h_{j} \frac{k_{1} h_{1}}{\left(\nu, q_{1}\right) r}\right)_{p}=\left(h_{j} \frac{q_{1}}{\left(\nu, q_{1}\right)}\right)_{p} .
$$

Finally, (8.7.3) follows from (3.1.2) while (8.7.4) was spelled out in the Addendum to (3.2).

Lemma 8.8. Whether or not $r_{p} \geq s_{p}$, at a prime $p$ so that $\nu \not \equiv 0 \bmod \left(q_{1}\right)_{p}$, the classes $\zeta_{2 j}$ and $c_{x}$ enjoy the following relations:

(1) In the typical case

$$
\begin{gathered}
\frac{s}{f_{p}} h_{j} \zeta_{2 j}=0 \\
\frac{\left(1+\left(t^{j}\right)+\cdots+\left(t^{j}\right)^{s-1}\right) h_{j}}{r} c_{x} \zeta_{2 j}=0 .
\end{gathered}
$$

(2) In the exceptional case

$$
2 \zeta_{2 j}=0 \text {, if } j \text { is odd, }
$$

$$
\frac{2 s}{(r, s)} h_{j} \zeta_{2 j}=0 \text {, if } j \text { is even and } t+1 \not \equiv 0 \bmod r_{2} \text {, }
$$

(8.10.e.1) $\frac{N(t, j, s) h_{j}}{r} c_{x} \zeta_{2 j}=0$, if $j$ is even,

$$
2 c_{x} \zeta_{2 j}=0 \text {, if } j \text { is odd, }
$$

where we have written $N(t, j, s)=1+t^{j}+\cdots+t^{j(s-1)}$ for short.

For clarity we note that (8.10.t) and (8.10.e.1) are equivalent to

$$
\frac{s_{p}}{d_{p}}\left(j, d_{p}\right) c_{x} \zeta_{2 j}=0
$$

Proof of (8.8). It is easy to see that the relations (8.9) hold. In fact, we have already observed that $\tilde{h}_{j}=r, \tilde{\nu}=f$, and $\left(\tilde{q}_{1}, \tilde{\nu}\right)=(r, \tilde{s}, f)$. Hence (4.7) entails $\frac{r \tilde{s}}{(r, \tilde{s}, f)} \tilde{\zeta}_{2 j}=0$ for $j \geq 1$, and it is clear that at the prime $p$ this may be written

$$
\frac{\tilde{s}_{p}}{\tilde{f}_{p}} \tilde{h}_{j} \tilde{\zeta}_{2 j}=0, \quad j \geq 1
$$

Hence the relation (8.9) holds at the start of the induction. We now suppose by induction that, in the typical case, (8.9.t) holds for $\mathbf{e}^{\prime}$, i.e., that $\frac{s^{\prime}}{f_{p}} h_{j}^{\prime} \zeta_{2 j}^{\prime}=0$ for $j \geq 1$. If $1 \leq j<d_{p}$, in view of (3.3), $\frac{s^{\prime}}{f_{p}} h_{j}^{\prime}=\frac{s}{f_{p}} h_{j}$, whence by construction (cf. $(4.15))$

$$
\frac{s}{f_{p}} h_{j} \zeta_{2 j}=\frac{s^{\prime}}{f_{p}} h_{j}^{\prime} \tau_{G^{\prime} \uparrow G}\left(\zeta_{2 j}^{\prime}\right)=0, \quad j \geq 1 .
$$


Consequently (8.9.t) holds in this range. However, if $j=d_{p}$, still in view of (3.3), $\left(h_{j}\right)_{p}=\left(h_{j}^{\prime}\right)_{p}$, whence taking into account (4.19), we conclude

$$
0=\frac{s_{p}^{\prime}}{f_{p}} h_{j}^{\prime} \tau_{G^{\prime} \uparrow G}\left(\zeta_{2 j}^{\prime}\right)=\frac{s_{p}^{\prime}}{f_{p}} h_{j} p \zeta_{2 j}=\frac{s_{p}}{f_{p}} h_{j} \zeta_{2 j}
$$

Hence (8.9.t) holds for $1 \leq j \leq d_{p}$.

In the exceptional case we have, by construction, $2 \zeta_{2 j}=0$ for $1 \leq j<d_{2}$ and $j$ odd, since these classes lie in the image of the modulo 2 Bockstein map (cf. their construction in $\S 4$ ), whence the relations (8.9.e.1) and (8.10.e.2). To handle the exceptional case for $j$ even we recall that the restricted case $\mathbf{e}^{\prime}$ is typical. If in addition $r_{2} \geq s_{2}$, in view of (3.4) the extension $\mathbf{e}^{\prime}$ splits at 2 , and by (2.8) we know that, for $j \geq 1, h_{j}^{\prime} \zeta_{2 j}^{\prime}=0$; for $1 \leq j \leq d_{2}$ we can then conclude, in view of (3.4) and (4.19), that at the prime 2 ,

$$
0=h_{j}^{\prime} \tau_{G^{\prime} \uparrow G}\left(\zeta_{2 j}^{\prime}\right)= \begin{cases}\left(t+1, \frac{r}{2}\right) h_{j} \tau_{G^{\prime} \uparrow G}\left(\zeta_{2 j}^{\prime}\right), & \text { if } j \text { is odd, } \\ 2 h_{j} \zeta_{2 j}, & \text { if } j \text { is even. }\end{cases}
$$

Likewise, in view of what has already been proved in the typical case, for $j \geq 1$, we have $\frac{s_{2}^{\prime}}{f_{2}} h_{j}^{\prime} \zeta_{2 j}^{\prime}=0$; furthermore, it is clear that $f_{2}=\frac{r_{2}}{2}$. For $1 \leq j \leq d_{2}$ we can then conclude, in view of (3.4) and (4.19), that

$$
0=\frac{s}{r} h_{j}^{\prime} \tau_{G^{\prime} \uparrow G}\left(\zeta_{2 j}^{\prime}\right)= \begin{cases}\frac{s}{r}\left(t+1, \frac{r}{2}\right) h_{j} \tau_{G^{\prime} \uparrow G}\left(\zeta_{2 j}^{\prime}\right), & \text { if } j \text { is odd } \\ 2 \frac{s}{r} h_{j} \zeta_{2 j}, & \text { if } j \text { is even. }\end{cases}
$$

In particular, (8.9.e.2) holds for $j \leq d_{2}$. If $t+1 \equiv 0 \bmod r_{2}$, in view of (3.4) the extension $\mathbf{e}^{\prime}$ splits at 2 and hence, in view of (2.8), $h_{2}^{\prime} \zeta_{4}^{\prime}=0$. Exploiting (3.4) and (4.19) again, we see that

$$
0=h_{2}^{\prime} \tau_{G^{\prime} \uparrow G}\left(\zeta_{4}^{\prime}\right)=2 h_{2} \zeta_{4}
$$

Hence the relations (8.9.e.3) hold for $j=2$. However, it is manifest that $d_{2}=2$, whence we have again verified these relations for $1 \leq j \leq d_{p}$. Finally, again an argument involving the multiplicative structure completes the proof that the relations (8.9) hold. We leave the details to the reader.

In order to verify the relations $(8.10)$ we proceed as follows: We take the obvious free presentation

$$
0 \rightarrow \mathbf{Z} \stackrel{s}{\longrightarrow} \mathbf{Z} \stackrel{\text { pr }}{\longrightarrow} K \longrightarrow 1
$$


and consider the pull back group $\hat{G}$ in the sense that the diagram

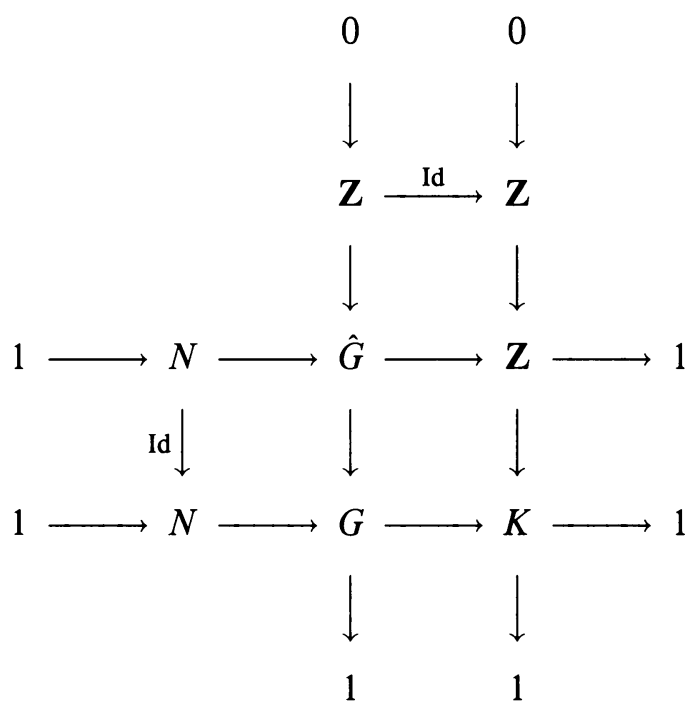

is commutative with exact rows and columns. For convenience we write

$$
\hat{\mathbf{e}}: 1 \rightarrow N \rightarrow \hat{G} \stackrel{\mathrm{pr}}{\longrightarrow} \mathbf{Z} \rightarrow 1
$$

for the pull back extension. The integral cohomology of $\hat{G}$ is readily computed from $\hat{\mathbf{e}}$. In fact, for degree reasons it is clear that

$$
\begin{aligned}
\mathbf{H}^{2 j}(\hat{G}, \mathbf{Z}) & =\mathbf{H}^{0}\left(\mathbf{Z}, \mathbf{H}^{2 j}(N, \mathbf{Z})\right)=\mathbf{Z} / h_{j}, \quad j \geq 1, \\
\mathbf{H}^{1}(\hat{G}, \mathbf{Z}) & =\mathbf{H}^{1}\left(\mathbf{Z}, \mathbf{H}^{0}(N, \mathbf{Z})\right)=\mathbf{Z}, \\
\mathbf{H}^{2 j+1}(\hat{G}, \mathbf{Z}) & =\mathbf{H}^{1}\left(\mathbf{Z}, \mathbf{H}^{2 j}(N, \mathbf{Z})\right)=\mathbf{Z} / h_{j}, \quad j \geq 1,
\end{aligned}
$$

and again for degree reasons there is not even a multiplicative extension problem.

We now consider the resulting central extension

$$
0 \rightarrow \mathbf{Z} \rightarrow \hat{G} \rightarrow G \rightarrow 1 \text {. }
$$

By construction it is clear that its class in $\mathrm{H}^{2}(G, \mathbf{Z})$ is just $c_{x} \in \mathrm{H}^{2}(G, \mathbf{Z})$. Hence part of the Gysin sequence of (8.15) looks like

$$
\mathrm{H}^{2 j+1}(\hat{G}) \stackrel{\vartheta_{2 j+1}}{\longrightarrow} \mathrm{H}^{2 j}(G) \stackrel{c_{x}}{\longrightarrow} \mathrm{H}^{2 j+2}(G) \stackrel{\mathrm{inf}}{\longrightarrow} \mathrm{H}^{2 j+2}(\hat{G}) \stackrel{\vartheta_{2 j+2}}{\longrightarrow} \mathrm{H}^{2 j+1}(G),
$$

where we have written $\mathrm{H}^{2 j}(G)=\mathrm{H}^{2 j}(G, \mathbf{Z})$, etc.

Lemma 8.17. Under the circumstances of (8.8) the following are true:

(1) For $j \geq 1, \inf \left(\zeta_{2 j}\right) \in \mathrm{H}^{2 j}(\hat{G}, \mathbf{Z})$ is a generator, whence, for $j \geq 0$, the morphism

$$
\vartheta_{2 j}: \mathrm{H}^{2 j}(\hat{G}, \mathbf{Z}) \rightarrow \mathrm{H}^{2 j-1}(G, \mathbf{Z})
$$

in the Gysin sequence (8.16) is zero. 
(2) For $j \geq 0$, for a suitable generator $\hat{\xi}_{2 j+1} \in \mathrm{H}^{2 j+1}(\hat{G}, \mathbf{Z})$,

$$
\vartheta_{2 j+1}\left(\hat{\xi}_{2 j+1}\right)=\frac{\left(1+\left(t^{j}\right)+\cdots+\left(t^{j}\right)^{s-1}\right) h_{j}}{r} \zeta_{2 j},
$$

unless we are in the exceptional case with $j$ odd.

Remark 8.17.3. The proof to be given below will also show that, if in the exceptional case $\nu \not \equiv 0 \bmod \left(q_{1}\right)_{2}$ and $t+1 \not \equiv 0 \bmod r_{2}$, for $j$ odd,

$$
\left(\frac{\left(1+\left(t^{j}\right)+\cdots+\left(t^{j}\right)^{s-1}\right) h_{j}}{r}\right)_{2}=\left(\frac{s(t+1)}{r}\right)_{2}=\left(q_{j}^{\prime}\right)_{2} .
$$
and

Notice if $G$ is abelian, i.e., if $t \equiv 1 \bmod r$, in view of (3.1), we have $h_{j}=r$

$$
\left(1+\left(t^{j}\right)+\cdots+\left(t^{j}\right)^{s-1}\right)=s,
$$

whence the statement of the lemma then says that, up to a unit modulo $p$,

$$
\vartheta_{2 j+1}\left(\hat{\xi}_{2 j+1}\right)=s \zeta_{2 j}
$$

Proof of the lemma. By construction, for $j \geq 1$,

$$
\inf \left(\zeta_{2 j}\right)=\frac{r}{h_{j}} c_{y}^{j} \in \mathrm{H}^{0}\left(\mathbf{Z}, \mathrm{H}^{2 j}(N, \mathbf{Z})\right)=\mathrm{H}^{2 j}(\hat{G}, \mathbf{Z}),
$$

and we know that this class is a generator; hence the morphism $\vartheta_{2 j}$ in the Gysin sequence (8.16) is then zero. We now concentrate on the proof of the other statement.

Assume first that $d=1$, i.e., that $G$ is abelian. Then $\hat{G}$ is abelian as well, and it is clear that the morphism $\vartheta_{1}$ in the Gysin sequence (8.16) sends a generator $\hat{\xi}_{1}$ of $\mathrm{H}^{1}(\hat{G}, \mathbf{Z}) \cong \mathbf{Z}$ to $s \in \mathbf{Z}=\mathrm{H}^{0}(G, \mathbf{Z})$. Since the Gysin sequence (8.16) is compatible with the obvious $\left(\mathrm{H}^{*}(G, \mathbf{Z})\right)$-module structures, we can conclude that, for $j \geq 1$, the morphism $\vartheta_{2 j+1}$ sends the generator $\hat{\xi}_{2 j+1}=\hat{\xi}_{1} \zeta_{2 j}$ of $\mathrm{H}^{2 j+1}(\hat{G}, \mathbf{Z})$ to

$$
\vartheta_{2 j+1}\left(\hat{\xi}_{2 j+1}\right)=s \zeta_{2 j} \in \mathrm{H}^{2 j}(G, \mathbf{Z}) .
$$

We now suppose $d_{p}>1$ and consider the extension $\mathbf{e}^{\prime}$. Let $0 \leq j \leq d_{p}$, and assume by induction that, for a suitable generator $\hat{\xi}_{2 j+1}^{\prime} \in \mathrm{H}^{2 j+1}\left(\hat{G}^{\prime}, \mathbf{Z}\right)$,

$$
\vartheta_{2 j+1}^{\prime}\left(\hat{\xi}_{2 j+1}^{\prime}\right)=\frac{\left(1+\left(t^{\prime j}\right)+\cdots+\left(t^{\prime j}\right)^{s^{\prime}-1}\right) h_{j}^{\prime}}{r} \zeta_{2 j}^{\prime} .
$$

Using the description $\mathrm{H}^{2 j+1}(\hat{G}, \mathbf{Z})=\mathrm{H}^{1}\left(\mathbf{Z}, \mathrm{H}^{2 j}(N, \mathbf{Z})\right)=\mathbf{Z} / h_{j}$ it is easy to see that

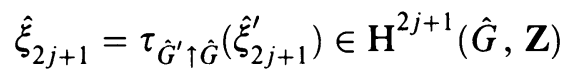


is a generator of $\mathrm{H}^{2 j+1}(\hat{G}, \mathbf{Z})$. Furthermore, since the transfer is natural, it is clear that

$$
\vartheta_{2 j+1}\left(\hat{\xi}_{2 j+1}\right)=\frac{\left(1+\left(t^{\prime j}\right)+\cdots+\left(t^{\prime j}\right)^{s^{\prime}-1}\right) h_{j}^{\prime}}{r} \tau_{G^{\prime} \uparrow G}\left(\zeta_{2 j}^{\prime}\right) .
$$

Our next aim is to calculate the coefficient $\frac{\left(1+\left(t^{j}\right)+\cdots+\left(t^{\prime j}\right)^{s^{\prime}-1}\right) h_{j}^{\prime}}{r}$ in terms of the undashed data: In the typical case we have, in view of (3.1) and (3.3),

$$
\left(\frac{\left(1+\left(t^{j}\right)+\cdots+\left(t^{j}\right)^{s^{\prime}-1}\right) h_{j}^{\prime}}{r}\right)_{p}=\left(\frac{\frac{s}{p} h_{j}^{\prime}}{r}\right)_{p}
$$

and

$$
\left(\frac{\frac{s}{p} h_{j}^{\prime}}{r}\right)_{p}= \begin{cases}\left(\frac{s h_{j}}{r}\right)_{p}=\left(\frac{\left(1+\left(t^{j}\right)+\cdots+\left(t^{j}\right)^{s-1}\right) h_{j}}{r}\right)_{p}, & \text { if } j \not \equiv 0 \bmod d_{p}, \\ \left(\frac{s h_{j}}{r p}\right)_{p}=\left(\frac{\left(1+\left(t^{j}\right)+\cdots+\left(t^{j}\right)^{s-1}\right) h_{j}}{r p}\right)_{p}, & \text { if } j \equiv 0 \bmod d_{p} .\end{cases}
$$

In view of (4.19) this implies that, up to a unit modulo $p$, for $0 \leq j \leq d_{p}$,

$$
\vartheta_{2 j+1}\left(\hat{\xi}_{2 j+1}\right)=\frac{\left(1+\left(t^{j}\right)+\cdots+\left(t^{j}\right)^{s-1}\right) h_{j}}{r} \zeta_{2 j} .
$$

Likewise, in the exceptional case, we recall that the restricted case $\mathrm{e}^{\prime}$ is typical (and splits); hence we have, in view of (3.1) and (3.3),

$$
\left(\frac{\left(1+\left(t^{j}\right)+\cdots+\left(t^{j}\right)^{s^{\prime}-1}\right) h_{j}^{\prime}}{r}\right)_{2}=\left(\frac{\frac{s}{2} h_{j}^{\prime}}{r}\right)_{2}
$$

and

$$
\left(\frac{\frac{s}{2} h_{j}^{\prime}}{r}\right)_{2}= \begin{cases}\left(\frac{s\left(t+1, \frac{r}{2}\right)}{r}\right)_{2}, & \text { if } j \text { is odd } \\ \left(\frac{s h_{j}}{2 r}\right)_{2}, & \text { if } j(t+1) \equiv 0 \bmod r_{2} \\ \left(\frac{s h_{j}}{r}\right)_{2}, & \text { otherwise. }\end{cases}
$$


However, in view of (3.1) and (3.4),

$$
\begin{aligned}
& \left(\frac{\left(1+\left(t^{j}\right)+\cdots+\left(t^{j}\right)^{s-1}\right) h_{j}}{r}\right)_{2}=\left(\frac{t^{j s}-1}{t^{j}-1} \frac{h_{j}}{r}\right)_{2} \\
& \quad= \begin{cases}\left(\frac{j s(t+1)}{j(t+1)} \frac{h_{j}}{r}\right)_{2}=\left(\frac{s h_{j}}{r}\right)_{2}, & \text { if } j \text { is even, } \\
\left(\frac{j s(t+1)}{2} \frac{h_{j}}{r}\right)_{2}=\left(\frac{s(t+1)}{r}\right)_{2}, & \text { if } j \text { is odd. }\end{cases}
\end{aligned}
$$

Consequently, if we exclude the case where $t+1 \equiv 0 \bmod r_{2}$ and $j$ is odd,

$$
\begin{aligned}
& \left.\left(\frac{\left(1+\left(t^{\prime j}\right)+\cdots+\left(t^{\prime j}\right)^{s^{\prime}-1}\right) h_{j}^{\prime}}{r}\right)_{2}\right)_{2}, \text { if } j(t+1) \equiv 0 \bmod r_{2}, \\
& \quad= \begin{cases}\left(\frac{\left(1+\left(t^{j}\right)+\cdots+\left(t^{j}\right)^{s-1}\right) h_{j}}{2 r}\right)_{2}, & \text { otherwise, } \\
\left(\frac{\left(1+\left(t^{j}\right)+\cdots+\left(t^{j}\right)^{s-1}\right) h_{j}}{r}\right)^{2} & \end{cases}
\end{aligned}
$$

since $\left(t+1, \frac{r}{2}\right)_{2}=(t+1)_{2}$. In view of (4.19) this implies that, up to a unit modulo 2 , for $0 \leq j \leq d_{2}$ and $j$ even,

$$
\vartheta_{2 j+1}\left(\hat{\xi}_{2 j+1}\right)=\frac{\left(1+\left(t^{j}\right)+\cdots+\left(t^{j}\right)^{s-1}\right) h_{j}}{r} \zeta_{2 j} .
$$

Moreover, we have also verified (8.17.4).

Finally, we observe that at $p$ the class $\inf \left(\zeta_{2 d_{p}}\right) \in \mathrm{H}^{2 d}(\hat{G}, \mathbf{Z})$ has order $r_{p}$, and that the Gysin sequence (8.16) is compatible with the obvious $\left(\mathrm{H}^{*}(G, \mathrm{Z})\right)$ module structures. It is straightforward to deduce from this that, for $j>d_{p}$, the morphism $\vartheta_{2 j+1}: \mathrm{H}^{2 j+1}(\hat{G}, \mathbf{Z}) \rightarrow \mathrm{H}^{2 j}(G, \mathbf{Z})$ in the Gysin sequence (8.16) has the asserted properties. We leave the details to the reader. This completes the proof of (8.17).

It is clear that Lemma 8.17 and the exactness of the Gysin sequence (8.16) imply at once that the relations (8.10) in (8.8) hold unless we are in the exceptional case with $j$ odd. However, we have already seen that the relations (8.9.e.1) and (8.10.e.2) hold. Hence the proof of (8.8) is now complete.

We now verify the relations (0.3.2) in the typical case. Before doing so we note that it may be necessary to rechoose some of the even degree generators $\zeta_{2 j}$. We assume that $p$ is a prime so that $r_{p} \geq s_{p}$ and, in view of (2.8) and (2.15), we assume as before that $\nu \not \equiv 0 \bmod \left(q_{1}\right)_{p}$ and concentrate at this prime. We 
know from (6.9) and (6.11) that, when $o c_{x} \zeta_{2 j}=0$, the number $o$ is divisible by $q_{j}$ at $p$. Since $r_{p} \geq s_{p}$, by (8.7) we have

$$
\left(1+\left(t^{j}\right)+\cdots+\left(t^{j}\right)^{s-1}\right)_{p}=\left(1+\left(t^{j}\right)+\cdots+\left(t^{j}\right)^{s-1}, r\right)_{p}=\left(k_{j}\right)_{p},
$$

unless we are in the exceptional case with $j$ odd, and the assertion is then a consequence of the fact that the relations (8.10.t) in (8.8) hold.

Next we verify the relations $(0.3 .1)$ in the typical case. We proceed as follows: Since we already proved that the relations $(0.3 .3)$ hold at the prime $p$, we know that, for $j \geq 1$,

$$
c_{x} \cdot \frac{q_{j-1}}{q_{1}}\left(q_{1}, \nu\right) c_{x} \zeta_{2 j-2}=0
$$

Now

$$
\frac{h_{j-1} f}{r}=h_{j-1} \frac{\nu r / h_{1}}{r}=\nu \frac{h_{j-1}}{h_{1}}=\nu \frac{q_{j-1}}{q_{1}}
$$

but the classes $\frac{q_{j-1}}{q_{1}}\left(\nu, q_{1}\right) c_{x} \zeta_{2 j-2}$ are nonzero in $\mathrm{H}^{2 j}(G, \mathbf{Z})$ since they pass to the classes $\frac{q_{j-1}}{q_{1}}\left(\nu, q_{1}\right) c_{x} c_{2 j-2}$ in $E_{\infty}$ (cf. (4.22)), and in view of (6.9) and (6.11) the latter are nonzero. The exactness of the Gysin sequence (8.16) implies then that the classes $\frac{q_{j-1}}{q_{1}}\left(\nu, q_{1}\right) c_{x} \zeta_{2 j-2}$ lie in the image of the morphism $\vartheta_{2 j+1}: \mathrm{H}^{2 j+1}(\hat{G}, \mathbf{Z}) \rightarrow \mathrm{H}^{2 j}(G, \mathbf{Z})$ in the Gysin sequence (8.16). Since $r_{p} \geq s_{p}$, in view of (8.7.1) and Lemma 8.17, we can conclude that, at the prime $p$, for suitable numbers $\alpha_{j}$,

$$
\frac{h_{j-1} f}{r} c_{x} \zeta_{2 j-2}=\alpha_{j} q_{j} \zeta_{2 j}
$$

The class $\zeta_{2 j}$ passes to the class $c_{2 j}$ in $E_{\infty}$ and by (6.9) and (6.11) the class $c_{2 j}$ has exact order $h_{j}$. Hence we must have $\alpha_{j} q_{j}=\alpha_{j}^{\prime} h_{j}$ at $p$. Furthermore, in view of what has already been established at $p$, the class $c_{x} \zeta_{2 j-2}$ has exact order $q_{j-1}$, whence the class

$$
\frac{h_{j-1} f}{r} c_{x} \zeta_{2 j-2}=\alpha_{j}^{\prime} h_{j} \zeta_{2 j}
$$

has exact order

$$
q_{j-1} / \frac{q_{j-1}}{q_{1}} \nu=\frac{q_{1}}{\left(\nu, q_{1}\right)}
$$

at $p$. Consequently, the class $h_{j} \zeta_{2 j}$ has at least order $\frac{q_{1}}{\left(\nu, q_{1}\right)}$ and hence the class $\zeta_{2 j}$ has at least order $h_{j} \frac{q_{1}}{\left(\nu, q_{1}\right)}$. However, by $(8.8)$, the class $\zeta_{2 j}$ has at most this order, whence $h_{j} \frac{q_{1}}{\left(\nu, q_{1}\right)}$ is the exact order of $\zeta_{2 j}$. Moreover, the numbers $\alpha_{j}$ are units at $p$. If necessary we can rechoose the classes $\zeta_{2 j}$ in such a way that these units assume the values 1 . This establishes the relations $(0.3 .1)$ in the typical case.

Finally we complete the exceptional case. We begin with the relations (0.3.3) for $j$ odd: In view of $(6.9)-(6.11)$, there is a priori a series of additional 
relations of the kind

$$
c_{x}^{i} \zeta_{2 j}=a_{1} c_{x}^{i+1} \zeta_{2 j-2}+a_{2} c_{x}^{i+2} \zeta_{2 j-4}+\cdots, \quad i \geq 2, j \text { odd }
$$

However, it is clear that if necessary we may rechoose the classes $\zeta_{2 j}$ for $j$ odd in such a way that

$$
c_{x}^{2} \zeta_{2 j}=0, \quad j \text { odd }
$$

and hence

$$
c_{x}^{i} \zeta_{2 j}=0, \quad i \geq 2, j \text { odd }
$$

This establishes the relations (0.3.3) in the exceptional case for $j$ odd.

In the exceptional case with $j$ even the relations $(0.3 .2)$ are settled in virtually the same way as in the typical case with (8.10.e.1) instead of (8.10.t). Moreover, for $j$ odd, in view of (8.19), in the Gysin sequence (8.16) the classes $c_{x} \zeta_{2 j} \in$ $\mathrm{H}^{2 j+2}(G)$ go to zero under multiplication by $c_{x}$; from the exactness of the Gysin sequence (8.16) we can therefore conclude that, in view of (8.7) and (8.17)(2), for suitable numbers $\alpha_{j+1}$, we must have

$$
c_{x} \zeta_{2 j}=\alpha_{j+1} q_{j+1} \zeta_{2 j+2} \text {. }
$$

Furthermore, in view of (6.9) and (6.11), the class $c_{x} \zeta_{2 j}$ has at least order 2 and the class $\zeta_{2 j+2}$ passes to the class $c_{2 j+2}$ in $E_{\infty}$ of order $h_{j+1}$. This implies that we must have a series of relations of the kind

$$
c_{x} \zeta_{2 j}=\alpha_{j+1}^{\prime} h_{j+1} \zeta_{2 j+2}
$$

and, in view of (8.9.e.2), the number $\alpha_{j+1}^{\prime}$ must be a unit at 2. In particular, we obtain the defining relations

$$
2 c_{x} \zeta_{2 j}=0, \quad j \text { odd }
$$

i.e., the relations $(0.3 .2)$ in the exceptional case with $j$ odd with the new choice for the generators $\zeta_{2 j}$. Moreover, we can replace $\zeta_{2 j+2}$ by $\alpha_{j+1}^{\prime} \zeta_{2 j+2}$ if necessary and henceforth write $\zeta_{2 j+2}$ for the latter; we then obtain the relations

$$
c_{x} \zeta_{2 j}=h_{j+1} \zeta_{2 j+2}, \quad j \text { odd. }
$$

Rewriting this we get

$$
h_{j} \zeta_{2 j}=c_{x} \zeta_{2 j-2}, \quad j \text { even, }
$$

i.e., the relations $(0.3 .1)$ for $j$ even.

Next, for $j$ odd, since $\left(h_{j}\right)_{2}=2$, in view of what was said in (6.9)-(6.11), we must have

$$
2 \zeta_{2 j}=a_{1} c_{x} \zeta_{2 j-2}+a_{2} c_{x}^{2} \zeta_{2 j-4}+\cdots, \quad j \text { odd }
$$

However, in view of what has already been established with the new choices for the $\zeta_{2 j}, 2 c_{x} \zeta_{2 j}=0$, whence

$$
0=a_{1} c_{x}^{2} \zeta_{2 j-2}+a_{2} c_{x}^{3} \zeta_{2 j-4}+\cdots, \quad j \text { odd }
$$


In view of what was said above we know that multiplication by $c_{x}$ is an isomorphism in filtration $\geq 4$. This implies that, in view of the structure of $\mathrm{H}^{4,2 j-2}(\mathbf{e})$ which we already know, for $k \geq 2$, the numbers $a_{k}$ are divisible by the orders of the classes $c_{x}^{k} \zeta_{2 j-2 k}$ and hence we must have relations of the kind

$$
2 \zeta_{2 j}=a_{1} c_{x} \zeta_{2 j-2} \in \mathrm{H}^{2 j}(G), \quad j \text { odd. }
$$

Furthermore, if the number $a_{1}$ is nonzero it is necessarily divisible by

$$
\left(\frac{q_{j-1}}{q_{1}}\right)_{2}=\frac{\left(q_{j-1}\right)_{2}}{2}
$$

since in view of what was just pointed out we must have

$$
0=a_{1} c_{x}^{2} \zeta_{2 j-2} \in \mathrm{H}^{2 j+2}(G), \quad j \text { odd. }
$$

Now for $j$ odd the element $\left(\frac{q_{j-1}}{q_{1}}\right)_{2} c_{x} \zeta_{2 j-2} \in \mathrm{H}^{2 j}(G)$ is manifestly nontrivial since it passes to the class

$$
\left(\frac{q_{j-1}}{q_{1}}\right)_{2} c_{x} c_{2 j-2} \in E_{\infty}^{2,2 j-2}(\mathbf{e})
$$

which is nontrivial. Hence the class

$$
\left(\frac{q_{j-1}}{q_{1}}\right)_{2} c_{x} \zeta_{2 j-2} \in \mathrm{H}^{2 j}(G)
$$

lies in the image of the morphism $\vartheta_{2 j+1}: \mathrm{H}^{2 j+1}(\hat{G}) \rightarrow \mathrm{H}^{2 j}(G)$ in the Gysin sequence (8.16). Since at 2 the group $\mathrm{H}^{2 j+1}(\hat{G})$ has order $\left(h_{j}\right)_{2}=2$ we conclude that the morphism $\vartheta_{2 j+1}$ is injective and that its image is generated by the class $\left(\frac{q_{j-1}}{q_{1}}\right)_{2} c_{x} \zeta_{2 j-2} \in \mathrm{H}^{2 j}(G)$. Furthermore, since we already know that, for $j$ odd, $2 c_{x} \zeta_{2 j}=0$, in view of the exactness of the Gysin sequence (8.16) we conclude that, at the prime 2 ,

$$
\left(h_{j}\right)_{2} \zeta_{2 j}=\varepsilon\left(\frac{q_{j-1}}{q_{1}}\right)_{2} c_{x} \zeta_{2 j-2},
$$

with $\varepsilon=1$ or $\varepsilon=0$ according as $\zeta_{2 j}$ has order 2 or $>2$ at the prime 2 . We do not pursue the question which case occurs when. Whatever value $\varepsilon$ assumes, in the first case we are done while in the second case we replace $\zeta_{2 j}$ by $\zeta_{2 j}+\frac{q_{j-1}}{2 q_{1}} c_{x} \zeta_{2 j-2}$; we note that this makes sense since in view of $(3.2)(2)$ the number $q_{j-1}$ is divisible by 4 . We then obtain the missing relations (0.3.1). However, we may have to change the relations $(0.3 .3)$ accordingly as indicated in the statement of $(0.3)$.

An argument involving the multiplicative structure then completes the proof that, at a good prime $p$, for a suitable choice of the generators the relations in Theorem 0.3 are defining relations for the structure of $\mathrm{H}^{*}(G)$ as a module over $\mathrm{H}^{*}(K)$. 
Remark 8.27. It is clear that the above yields a complete description of the additive structure at the prime 2 in the exceptional case for $\nu$ odd, too. Indeed, for $j$ odd, write $Z_{2 j} \in \mathrm{H}^{2 j}(G)$ for the old generators constructed by means of the modulo 2 Bockstein exact sequence in $\S 2$. The above shows that, if we multiply the classes $\zeta_{2 j} \in \mathrm{H}^{2 j}(G)$ for $j$ even by appropriate units at 2 (if necessary) and write $Z_{2 j} \in \mathrm{H}^{2 j}(G)$ for the corresponding classes, at the prime 2 the cohomology $\mathrm{H}^{*}(G)$ is as a module over the 2-part of $\mathrm{H}^{*}(K)$ generated by

$$
Z_{2}, Z_{4}, \ldots, \xi_{5}, \xi_{9}, \ldots
$$

subject to the relations

$$
\begin{aligned}
2 Z_{2 j} & =0, & & j \text { odd, } \\
h_{j} Z_{2 j} & =c_{x} Z_{2 j-2}, & & j \text { even, } \\
\frac{q_{j}}{q_{1}} \xi_{2 j+1} & =0, & & j \text { even, } \\
\frac{q_{j}}{q_{1}} c_{x}^{i} Z_{2 j} & =0, & & j \text { even, } i \geq 2, \\
c_{x}^{i} Z_{2 j} & =\varepsilon \frac{q_{j-1}}{2 q_{1}} c_{x}^{i+1} Z_{2 j-2}, & & j \text { odd, } i \geq 2,
\end{aligned}
$$

where $\varepsilon=0$ or $\varepsilon=1$, but the latter ambiguity does not affect the additive structure.

\section{REFERENCES}

1. K. Alzubaidy, Metacyclic p-groups and Chern classes, Illinois J. Math. 26 (1982), 423-431.

2. M. André, Le $d_{2}$ de la suite spectrale en cohomologie de groupes, C. R. Acad. Sci. Paris Sér. A, B 260 (1965), 2669-2671.

3. $\_$Homologie des extensions de groupes, C. R. Acad. Sci. Paris Sér. A, B 260 (1965), 3820-3823.

4. F. R. Beyl, The Schur multiplicator of metacyclic groups, Proc. Amer. Math. Soc. 40 (1973), 413-418.

5. F. R. Beyl and J. Tappe, Group extensions, representations, and the Schur multiplicator, Lecture Notes in Math., vol. 958, Springer, Berlin, Heidelberg, and New York, 1982.

6. W. Browder, Cohomology and group actions, Invent. Math. 71 (1983), 599-607; Addendum and Erratum 75 (1984), 585.

7. R. Brown, The twisted Eilenberg-Zilber theorem, Celebrazioni Archimedee del Secolo XX, Simposio di Topologia (1964).

8. H. Cartan and S. Eilenberg, Homological algebra, Princeton Univ. Press, Princeton, N.J., 1956.

9. B. Cenkl and R. Porter, Polynomial cochains on nilmanifolds, Preprint, 1989.

10. L. S. Charlap and A. T. Vasquez, The cohomology of group extensions, Trans. Amer. Math. Soc. 124 (1966), 24-40.

11. Characteristic classes for modules over groups. I, Trans. Amer. Math. Soc. 127 (1969), 533-549. 
12. L. Evens and St. Priddy, The cohomology of the semi-dihedral group, Conference on Algebraic Topology in honor of P. Hilton (R. Piccinini and D. Sjerve, eds.), Contemporary Math., vol. 37, Amer. Math. Soc., Providence, R.I., 1985, pp. 61-72.

13. V. K. A. M. Gugenheim, On the chain complex of a fibration, Illinois J. Math. 16 (1972), 398-414.

14. 25 (1982), 197-205.

15. V. K. A. M. Gugenheim and L. Lambe, Perturbation in differential homological algebra, Illinois J. Math. 33 (1989), 566-582.

16. V. K. A. M. Gugenheim, L. Lambe, and J. D. Stasheff, Algebraic aspects of Chen's twisting cochains, Illinois J. Math. 34 (1990), 485-502.

17. _ Perturbation theory in differential homological algebra. II, Illinois J. Math. (to appear).

18. V. K. A. M. Gugenheim and J. P. May, On the theory and applications of differential torsion products, Mem. Amer. Math. Soc. No. 142 (1974).

19. V. K. A. M. Gugenheim and J. Milgram, On successive approximations in homological algebra, Trans. Amer. Math. Soc. 150 (1970), 157-182.

20. V. K. A. M. Gugenheim and H. J. Munkholm, On the extended functoriality of Tor and Cotor, J. Pure Appl. Algebra 4 (1974), 9-29.

21. V. K. A. M. Gugenheim and J. D. Stasheff, On perturbations and $A_{\infty}$-structures, Festschrift in honor of G. Hirsch's 60th birthday (L. Lemaire, ed.), Bull. Soc. Math. Belgique 38 (1986), 237-245.

22. P. J. Hilton and U. Stammbach, A course in homological algebra, Graduate Texts in Math., vol. 4, Springer, Berlin, Heidelberg, and New York, 1971.

23. G. Hochschild and J. P. Serre, Cohomology of group extensions, Trans. Amer. Math. Soc. 74 (1953), 110-134.

24. J. Huebschmann, Perturbation theory and free resolutions for nilpotent groups of class 2, J. Algebra 126 (1989), 348-399.

25. _ Cohomology of nilpotent groups of class 2, J. Algebra 126 (1989), 400-450.

26. Change of rings and characteristic classes, Math. Proc. Cambridge Philos. Soc. 106 (1989), 29-56.

27. Chern classes for metacyclic groups, Arch. Math. (to appear).

28. __ Change of rings and modules up to higher homotopies (in preparation).

29. _ Cohomology of solvable groups (in preparation).

30. 53-105.

31. The homotopy type of $F \Psi^{q}$. The complex and symplectic cases, Applications of Algebraic $K$-Theory to Algebraic Geometry and Number Theory, Part II (Proc. Conf. Boulder, Colorado, June 12-18, 1983), Contemporary Math., vol. 55, Amer. Math. Soc., Providence, R.I., 1986, pp. 487-518.

32. _ Perturbation theory and small models for the chains of certain induced fibre spaces, Habilitat. Univ. Heidelberg, 1984, Zbl 576.55012.

33. __ Minimal free multi models for chain algebras, Preprint, 1990.

34. J. Huebschmann and T. Kadeishvili, Small models for chain algebras, Math. Z. (to appear).

35. L. Lambe and J. D. Stasheff, Applications of perturbation theory to iterated fibrations, Manuscripta Math. 58 (1987), 363-376.

36. D. S. Larson, The integral cohomology rings of split metacyclic groups, Ph.D. Thesis, University of Minnesota, 1987.

37. M. Lazard, Groupes analytiques p-adiques, Inst. Hautes Études Sci. Publ. Math. 26 (1965), 389-603. 
38. G. Lewis, The integral cohomology rings of groups of order $p^{3}$, Trans. Amer. Math. Soc. 132 (1968), 501-529.

39. S. Mac Lane, Homology, Grundlehren Math. Wiss., No. 114, Springer, Berlin, Göttingen, and Heidelberg, 1963.

40. I. Madsen and J. Milgram, Classifying spaces for surgery and cobordism of manifolds, Ann. of Math. Studies, No. 92, Princeton Univ. Press, Princeton, N.J., 1979.

41. C. H. Sah, Cohomology of split group extensions, J. Algebra 29 (1974), 255-302; II, J. Algebra 45 (1977), 17-68.

42. M. Tezuka and N. Yagita, The varieties of the $\bmod p$ cohomology rings of extra special p-groups for an odd prime $p$, Math. Proc. Cambridge Philos. Soc. 94 (1983), 449-459.

43. C. B. Thomas, Chern classes and metacyclic p-groups, Mathematika 18 (1971), 196-200.

44. _ Chern classes of representations, Bull. London Math. Soc. 18 (1986), 225-240.

45. _ Characteristic classes and the cohomology of finite groups, Cambridge Studies in Advanced Math., vol. 9, Cambridge Univ. Press, Cambridge, 1986.

46. C. T. C. Wall, Resolutions for extensions of groups, Math. Proc. Cambridge Philos. Soc. 57 (1961), 251-255.

47. H. Zassenhaus, Lehrbuch der Gruppentheorie, Teubner Verlag, Leipzig, 1937; English transl., Chelsea, New York, 1949.

Mathematisches Institut, Im Neuenheimer Feld 288, D-6900 Heidelberg, Germany

Current address: UFR de Mathématiques, Université de Lille-Flandres-Artois, F-59655 Villeneuve D'Ascq Cedex, France

E-mail address: HUEBSCHM@FRCITL81.bitnet 